\title{
A NEW EQUIVALENCE BETWEEN SUPER HARISH-CHANDRA PAIRS AND LIE SUPERGROUPS
}

\author{
Fabio GAVARINI \\ Dipartimento di Matematica, Università di Roma "Tor Vergata" \\ via della ricerca scientifica 1 - I-00133 Roma, Italy \\ e-mail: gavarini@mat.uniroma2.it
}

\begin{abstract}
It is known that there exists a natural functor $\Phi$ from Lie supergroups to super Harish-Chandra pairs. A functor going backwards, that associates a Lie supergroup with each super Harish-Chandra pair, yielding an equivalence of categories, was found by Koszul [19], and later generalized by several authors.

In this paper, we provide two new backwards equivalences, i.e. two different functors $\Psi^{\circ}$ and $\Psi^{e}$ that construct a Lie supergroup (thought of as a special group-valued functor) out of a given super Harish-Chandra pair, so that both $\Psi^{\circ}$ and $\Psi^{e}$ are quasi-inverse to the functor $\Phi$.
\end{abstract}

\section{Introduction}

To every Lie supergroup $G$ one can associate the pair $\left(G_{\mathbf{0}}, \mathfrak{g}\right)$ formed by the classical Lie group $G_{\mathbf{0}}$ underlying $G$ and the tangent Lie superalgebra $\mathfrak{g}=\operatorname{Lie}(G)$ of $G$; these two objects are "compatible" in a natural sense, so that their pair is what is called a "super Harish-Chandra pair", or just "sHCp" for short. Overall, mapping $G \mapsto\left(G_{\mathbf{0}}, \mathfrak{g}\right)$ yields a functor, call it $\Phi$, from the category of Lie supergroups (either smooth, analytic or holomorphic) to the category of super Harish-Chandra pairs - of smooth, analytic or holomorphic type respectively. Is there any functor $\Psi$ from sHCp's to Lie supergroups which be a quasiinverse for $\Phi$, so that the two categories be equivalent? And how much explicit such a functor (if any) is?

A first answer to this question was given by Kostant and by Koszul in the real smooth case (see [18] and [19]), providing an explicit quasi-inverse for $\Phi$. Later on, Vishnyakova (see [25]) fixed the complex holomorphic case, and her proof works for the real analytic case as well. More recently, this result was increasingly extended to the setup of algebraic supergeometry (see [8], [21], [22]). It is worth remarking, though, that all these subsequent results were, in the end, further improvements of the original idea by Koszul (while Kostant's method was a slight variation of that), who defined a Lie supergroup out of a sHCp $\left(K_{+}, \mathfrak{k}\right)$ as a super-ringed space, defining the "proper" sheaf of superalgebras onto $K_{+}$by means of $\mathfrak{k}$.

In this paper we present a new solution, namely we provide a new functor $\Psi$ - in two different versions - from sHCp's to Lie supergroups that is quasi-inverse to $\Phi$. For this we follow the approach where, instead of thinking of supermanifolds as being super-ringed manifolds, one treats them as suitable functors, defined on the category of "Weil superalgebras". This point of view allows to unify several different approaches to supergeometry (see [3]) and also to treat the infinite-dimensional setup (see [2]); for a broader discussion about this, we refer to classical sources as [4], [10], [20], [24] or more recent ones like [3], [5], [7], [23].

Now, if we want a functor $\Psi$ from sHCp's to Lie supergroups, we need a Lie supergroup $G_{\mathcal{P}}$ for each sHCp $\mathcal{P}$; to have such a $G_{\mathcal{P}}$ (as a functor) we need a Lie group $G_{\mathcal{P}}(A)$ for each Weil superalgebra $A$,

$2020 M S C$ : Primary 58A50, 14M30; Secondary 17B99

Keywords: Lie supergroups; Lie superalgebras; super Harish-Chandra pairs.

Partially supported by the MIUR Excellence Department Project awarded to the Department of Mathematics, University of Rome "Tor Vergata", CUP E83C18000100006. 
whose definition must be natural in $A$ : moreover, one still has to show that the resulting functor have those additional properties that make it into a Lie supergroup. Finally, all this should aim to find a $\Psi$ that is quasi-inverse to $\Phi$ - and this fixes ultimate bounds to the construction we aim to.

Bearing all this in mind, the construction that we present goes as follows. Given a super Harish-Chandra pair $\mathcal{P}=\left(G_{+}, \mathfrak{g}\right)$, for each Weil superalgebra $A$, we define a group $G_{\mathcal{P}}(A)$ abstractly, by generators and relations: this definition is natural in $A$, hence it yields a functor from Weil algebras to (abstract) groups, call it $G_{\mathcal{P}}$ - cf. $\S 3.1$ and $\S 3.3$. As a key step in the work, we prove that $G_{\mathcal{P}}$ admits a "global splitting", i.e. it is the direct product of $G_{+}$times a totally odd affine superspace (isomorphic to $\mathfrak{g}_{1}$, the odd part of $\mathfrak{g})$ : as both these are supermanifolds, it turns out that $G_{\mathcal{P}}$ itself is a supermanifold as well, hence it is a Lie supergroup because (as a functor) it is group-valued too - cf. $\S 3.2$ and $\S 3.4$. One more step proves that the construction of $G_{\mathcal{P}}$ is natural in $\mathcal{P}$, so it yields a functor $\Psi$ from sHCp's to Lie supergroups: this is our candidate to be a quasi-inverse to $\Phi$ - cf. Theorem 3.2.6 and Theorem 3.4.6.

It is immediate to check that $\Phi \circ \Psi$ is isomorphic to the identity functor onto sHCp's, while proving that $\Psi \circ \Phi$ is isomorphic to the identity on Lie supergroups is much more demanding. For this we need to know that every Lie supergroup $G$ has a "global splitting" on its own: this fact is more or less known among specialists, but we need it stated in a genuine geometrical form, while it is usually given in sheaf-theoretic terms - so we work it out explicitly (cf. §2.4). In fact, we find two different formulations of such a result: this is why, building upon them, we can provide two versions, $\Psi^{\circ}$ and $\Psi^{e}$, of a functor $\Psi$ as required.

Finally, the reader can find a more detailed treatment in the expanded version [17] of this paper. Moreover, specific examples of application can be realized by suitably adapting the constructions of algebraic supergroups presented in [11], [12], [13], [14] and [15].

\section{Acknowledgements}

The author thanks Alexander Alldridge, Claudio Carmeli and Rita Fioresi for many valuable conversations.

\section{Preliminaries}

Hereafter we fix notation and terminology for super mathematics, referring to the literature (e.g. [1], [2], [7], [11], [24]) for further details. We will denote by $\mathbb{K}$ the field $\mathbb{R}$ or $\mathbb{C}$ of real or complex numbers.

\subsection{Recollection and notation}

2.1.1. Supermodules, superalgebras, Lie superalgebras. We denote by $(\mathbf{s m o d})_{\mathbb{K}}$, resp. $(\bmod )_{\mathbb{K}}$, the category of $\mathbb{K}$-supermodules, resp. $\mathbb{K}$-modules, and by $(\mathbf{s a l g})_{\mathbb{K}}$, resp. (alg $)_{\mathbb{K}}$ — possibly dropping $\mathbb{K}$ - the category of all commutative $\mathbb{K}$-superalgebras, resp. $\mathbb{K}$-algebras. We write $\mathbb{Z}_{2}=\{\mathbf{0}, \mathbf{1}\}$ for the group with two elements and $|x|$ or $p(x)\left(\in \mathbb{Z}_{2}\right)$ for the parity of any homogeneous $x$ in some superspace. For $A \in($ salg $)$, $n \in \mathbb{N}$, we call $A_{\mathbf{1}}^{[n]}$ the $A_{\mathbf{0}}$-submodule of $A$ spanned by all products $\vartheta_{1} \cdots \vartheta_{n}$ with $\vartheta_{i} \in A_{\mathbf{1}}$ for all $i$. If $J_{A}:=\left(A_{\mathbf{1}}\right)$ is the ideal of $A$ generated by $A_{\mathbf{1}}$, then $J_{A}=A_{1}^{[2]} \oplus A_{\mathbf{1}}$, and $\bar{A}:=A / J_{A}$ is a commutative superalgebra which is totally even, i.e. $\bar{A} \in(\mathbf{a l g})$, with an obvious isomorphism $\bar{A}:=A /\left(A_{\mathbf{1}}\right) \cong A_{\mathbf{0}} / A_{\mathbf{1}}^{[2]}$.

We call Weil superalgebra any finite-dimensional commutative $\mathbb{K}$-superalgebra $A$ such that $A=\mathbb{K} \oplus A$ where $\mathbb{K}$ is even and $\stackrel{\AA}{\AA}=\AA_{\mathbf{0}} \oplus \stackrel{\circ}{A}_{\mathbf{1}}$ is the nilradical of $A$ (cf. [3] and references therein). Every Weil superalgebra $A$ is endowed with the canonical morphisms $p_{A}: A \longrightarrow \mathbb{K}$ and $u_{A}: \mathbb{K} \longrightarrow A$ associated with the direct sum splitting $A=\mathbb{K} \oplus \AA$; thus $p_{A} \circ u_{A}=i d_{\mathbb{K}}$, so $p_{A}$ is surjective and $u_{A}$ is injective. Weil superalgebras over $\mathbb{K}$ form a full subcategory of $(\mathbf{s a l g})_{\mathbb{K}}$, denoted $(\text { Wsalg })_{\mathbb{K}}$ or just $($ Wsalg $)$.

We denote by $(\mathbf{s L i e})_{\mathbb{K}}$, or just $(\mathbf{s L i e})$, the category of all Lie $\mathbb{K}$-superalgebras. For any $\mathfrak{g} \in(\mathbf{s L i e})_{\mathbb{K}}$, we use notation $Y^{\langle 2\rangle}:=2^{-1}[Y, Y]\left(\in \mathfrak{g}_{\mathbf{0}}\right)$ for $Y \in \mathfrak{g}_{\mathbf{1}}$. In particular, for $V \in(\text { smod })_{\mathbb{K}}$ we consider $\mathfrak{g l}(V):=\operatorname{End}(V)$ as a Lie $\mathbb{K}$-superalgebra, with Lie superbracket $[A, B]:=A B-(-1)^{|A||B|} B A$ for homogeneous $A, B \in \operatorname{End}(V)$; then $Y^{\langle 2\rangle}=Y^{2}$ for odd $Y$. If $V:=V_{\mathbf{0}} \oplus V_{\mathbf{1}}$ with $V_{\mathbf{0}}:=\mathbb{K}^{p}$ and $V_{\mathbf{1}}:=\mathbb{K}^{q}$ we write $\operatorname{End}\left(\mathbb{K}^{p \mid q}\right):=\operatorname{End}(V)$ or $\mathfrak{g l}_{p \mid q}:=\operatorname{End}(V)$. Every $\mathfrak{g} \in(\mathbf{s L i e})_{\mathbb{K}}$ defines a functor

$$
\mathcal{L}_{\mathfrak{g}}:(\mathbf{W s a l g})_{\mathbb{K}} \longrightarrow(\mathbf{L i e})_{\mathbb{K}}, \quad A \mapsto \mathcal{L}_{\mathfrak{g}}(A):=(A \otimes \mathfrak{g})_{\mathbf{0}}=\left(A_{\mathbf{0}} \otimes \mathfrak{g}_{\mathbf{0}}\right) \oplus\left(A_{\mathbf{1}} \otimes \mathfrak{g}_{\mathbf{1}}\right)
$$


where $(\text { Lie })_{\mathbb{K}}$ is the category of Lie $\mathbb{K}$-algebras and the Lie bracket in $A \otimes \mathfrak{g}$ is $\left[a \otimes X, a^{\prime} \otimes X^{\prime}\right]:=$ $(-1)^{|X|\left|a^{\prime}\right|} a a^{\prime} \otimes\left[X, X^{\prime}\right]$ (see [7] for details). In particular, all this applies to $\mathfrak{g}:=\operatorname{End}(V)$.

This functorial presentation applies in particular to representations: so, if $\phi: \mathfrak{g} \longrightarrow \operatorname{End}(V)$ is a representation of $\mathfrak{g}$ onto the $\mathfrak{g}$-module $V$, then it induces a natural transformation $\mathcal{L}_{\mathfrak{g}} \longrightarrow \mathcal{L}_{\operatorname{End}(V)}$.

2.1.2. Supermanifolds and supergroups. We consider "supermanifolds" (real smooth, real analytic or complex holomorphic ones) and the corresponding group objects (i.e. "Lie supergroups") as described in [3]. If $M$ is any supermanifold, we denote by $|M|$ its underlying topological space and by $\mathcal{O}_{M}$ its structure sheaf. Moreover, by $M_{\mathbf{0}}$ we denote the so-called reduced (classical) smooth manifold associated with $M$, that can be thought of as an embedded sub(super)manifold of $M$ itself. We denote the category of (real) smooth, (real) analytic, or (complex) holomorphic supermanifolds by (ssmfd), (asmfd), or (hsmfd), respectively. In most cases the distinction between these cases is immaterial, hence we shall often refer only to "supermanifolds".

Finally, by Lie supergroup (real smooth, real analytic or complex holomorphic) we mean a group object in the category of supermanifolds (of the same type). These objects, together with the obvious morphisms, form a subcategory among supermanifolds, denoted $(\text { Lsgrp })_{\mathbb{R}}^{\infty}$, resp. $(\text { Lsgrp })_{\mathbb{R}}^{\omega}$, resp. $(\text { Lsgrp })_{\mathbb{C}}^{\omega}$.

2.1.3. The functorial point of view. We now recall the language of "functor(s) of points", referring to [3] for details. For two categories $\mathbf{A}$ and $\mathbf{B}$, by $[\mathbf{A}, \mathbf{B}]$ we mean the category of all functors between $\mathbf{A}$ and $\mathbf{B}$, whose morphisms are the natural transformations, while $\mathbf{A}^{\mathrm{op}}$ will denote the opposite category to $\mathbf{A}$.

Given $M \in(\mathbf{s s m f d})$, its associated functor of points $\mathcal{F}_{M}:(\mathbf{s s m f d})^{\mathrm{op}} \longrightarrow(\mathbf{s e t})$ is defined on objects by $\mathcal{F}_{M}(S):=\operatorname{Hom}(S, M)$ and on morphisms by $\mathcal{F}_{M}(\phi): \mathcal{F}_{M}(S) \longrightarrow \mathcal{F}_{M}(T), f \mapsto\left(\mathcal{F}_{M}(\phi)\right)(f):=f \circ \phi$, for $S, T \in(\mathbf{s s m f d})$ and $\phi \in \operatorname{Hom}(S, T)$; the elements in $\mathcal{F}_{M}(S)$ are the "S-points of $M$ ". Given $M, N \in$ $(\mathbf{s s m f d})$, Yoneda's Lemma yields a full and faithful immersion $\mathcal{Y}:(\mathbf{s s m f d}) \longrightarrow\left[(\text { ssmfd })^{\text {op }}\right.$, (set $\left.)\right]$ given on objects by $M \mapsto \mathcal{F}_{M}$. Similar constructions hold with (asmfd), resp. (hsmfd), replacing (ssmfd) .

Following [3], we consider a variant of this construction, that of " $A$-points" of a supermanifold. To any $M \in(\mathbf{s s m f d})$ we associate its Weil-Berezin (local) "functor of $A$-points" $\mathcal{W}_{M}:(\mathbf{W s a l g}) \longrightarrow\left(\mathcal{A}_{\mathbf{0}^{-}} \mathbf{s m f d}\right)$ where $\left(\mathcal{A}_{\mathbf{0}^{-}}\right.$-smfd $)$is a suitable category of " $\mathcal{A}_{\mathbf{0}}$-manifolds". This construction is natural in $M$, hence it yields a functor $\mathcal{W}:(\mathbf{s s m f d}) \longrightarrow\left[\left[(\mathbf{W}\right.\right.$ salg $\left.\left.),\left(\mathcal{A}_{\mathbf{0}^{-}} \mathbf{- s m f d}\right)\right]\right]$, called Shvarts embedding, that is full and faithful. Similar constructions and results apply to analytic and to holomorphic supermanifolds as well.

Therefore one can correctly study supermanifolds via their Weil-Berezin functors. In particular, the Shvarts embedding $\mathcal{W}$ preserves products, hence also group objects: this implies (cf. [3], §4) that a supermanifold $M$ is a Lie supergroup if and only if $\mathcal{W}_{M}$ takes values in the subcategory (among $\mathcal{A}_{\mathbf{0}}-$ manifolds) of group objects (that we call "Lie $\mathcal{A}_{\mathbf{0}}$-groups").

\subsection{Lie supergroups and super Harish-Chandra pairs}

Hereafter we recall the notion of super Harish-Chandra pair (=sHCp in short), referring to [7] for details. 2.2.1. Super Harish-Chandra pairs. We call (smooth, analytic or holomorphic) super Harish-Chandra pair over $\mathbb{K}$ any pair $\left(G_{+}, \mathfrak{g}\right)$ such that: $\quad$ (a) $G_{+}$is a (smooth, etc.) Lie group over $\mathbb{K}, \quad$ (b) $\mathfrak{g} \in(\mathbf{s L i e})_{\mathbb{K}}$, (c) $\operatorname{Lie}\left(G_{+}\right)=\mathfrak{g}_{0}$ and there is an action $A d: G_{+} \longrightarrow \operatorname{Aut}(\mathfrak{g})$ which extends the adjoint action of $G_{+}$ on $\operatorname{Lie}\left(G_{+}\right)=\mathfrak{g}_{\mathbf{0}}$ and whose differential is the restriction to $\operatorname{Lie}\left(G_{+}\right)=\mathfrak{g}_{0}$ of the adjoint action of $\mathfrak{g}$. All super Harish-Chandra pairs over $\mathbb{K}$, with their obvious morphisms, form a category, denoted $(\mathbf{s} \mathbf{H} \mathbf{C p})_{\mathbb{K}}$ - or $(\mathbf{s H C p})_{\mathbb{R}}^{\infty},(\mathbf{s} \mathbf{H C p})_{\mathbb{R}}^{\omega}$ or $(\mathbf{s} \mathbf{H C p})_{\mathbb{C}}^{\omega}$ to specify that we are taking smooth, analytic or holomorphic objects.

2.2.2. The super Harish-Chandra pair of a Lie supergroup. Let $G$ be a Lie supergroup (of either type: smooth, etc.). It is known that its underlying reduced manifold $G_{\mathbf{0}}$ (cf. §2.1.2) is indeed a Lie group (either smooth, etc., like $G$ is). Even more, the construction $G \mapsto G_{\mathbf{0}}$ yields a functor from Lie supergroups (of either type) to Lie groups (of the same type). Moreover, $G$ has a tangent Lie superalgebra $\operatorname{Lie}(G)$, whose construction is natural in $G$ so to define a functor from Lie supergroups to Lie superalgebras (cf. [7]). In the sequel by $\operatorname{Lie}(G)$ we will mean both this Lie superalgebra and its associated functor as in $\S 2.1 .1$. Note that for $A \in(\mathbf{W} \text { salg })_{\mathbb{K}}$ one has $\operatorname{Lie}(G)(A)=\operatorname{Lie}(G(A))$, the latter being the tangent Lie algebra to the Lie group $G(A)$. Now $\left(G_{\mathbf{0}}, \operatorname{Lie}(G)\right)$ is a super Harish-Chandra pair - natural in $G$ - so we have functors

$$
\Phi:(\text { Lsgrp })_{\mathbb{R}}^{\infty} \longrightarrow(\mathbf{s H C p})_{\mathbb{R}}^{\infty}, \quad \Phi:(\text { Lsgrp })_{\mathbb{R}}^{\omega} \longrightarrow(\mathbf{s H C p})_{\mathbb{R}}^{\omega}, \quad \Phi:(\text { Lsgrp })_{\mathbb{C}}^{\omega} \longrightarrow(\mathbf{s H C p})_{\mathbb{C}}^{\omega}
$$

given on objects by $G \mapsto\left(G_{\mathbf{0}}, \operatorname{Lie}(G)\right)$ and on morphisms by $\phi \mapsto\left(\phi_{\mathbf{0}}, \operatorname{Lie}(\phi)\right)$. 


\subsection{Special splittings for Lie supergroups}

We remind now some special splittings (either "pointwise", say, or "global" ones) for Lie supergroups: we will then take them as a model to build up a Lie supergroup out of a given super Harish-Chandra pair.

2.3.1. Pointwise splittings for Lie supergroups. Given a Lie supergroup $G$, there exists a bunch of results concerning particular splittings (typically, as semidirect product) of its groups of $A$-points $G(A)$. They are more or less known, see e.g. cf. [6] or [23], but other authors too may have mentioned them.

To begin with, let $p: A^{\prime} \longrightarrow A^{\prime \prime}$ and $u: A^{\prime \prime} \longrightarrow A^{\prime}$ be morphisms in $(\mathbf{W s a l g})_{\mathbb{K}}$ such that $p \circ u=i d_{A^{\prime \prime}}$, and let $G:(\text { Wsalg })_{\mathbb{K}} \longrightarrow$ (group) be any functor. Then $G(A)$ canonically splits into a semi-direct product, namely $G(A)=\operatorname{Im}(G(u)) \ltimes \operatorname{Ker}(G(p)) \cong G\left(A^{\prime \prime}\right) \ltimes \operatorname{Ker}(G(p))$. In particular, when the functor $G$ is in fact a Lie supergroup, choose $A^{\prime}:=A \in(\mathbf{W} \text { salg })_{\mathbb{K}}, A^{\prime \prime}:=\mathbb{K}$ with $p:=p_{A}$ and $u:=u_{A}$ as in $\S 2.1 .1$. Then the splitting $G(A) \cong G\left(A^{\prime \prime}\right) \ltimes \operatorname{Ker}(G(p))$ given above now reads as a canonical splitting of Lie groups $G(A) \cong G_{\mathbf{0}}(\mathbb{K}) \ltimes N_{G}(A)$, where $G_{\mathbf{0}}(\mathbb{K})$ is the ordinary Lie group underlying $G$ and $N_{G}(A):=\operatorname{Ker}\left(G\left(p_{A}\right)\right)$.

2.3.2. Pointwise splitting for Lie superalgebras. Consider a Lie $\mathbb{K}$-superalgebra $\mathfrak{g}=\mathfrak{g}_{0} \oplus \mathfrak{g}_{1}$ and its associated functor $\mathcal{L}_{\mathfrak{g}}:(\mathbf{s a l g})_{\mathbb{K}} \longrightarrow(\text { Lie })_{\mathbb{K}}$ as in $\S 2.1 .1$. Acting like in 2.3.1, for any $A \in(\mathbf{W s a l g})_{\mathbb{K}}$ we have $\mathcal{L}_{\mathfrak{g}}(A)=\operatorname{Im}\left(\mathcal{L}_{\mathfrak{g}}\left(u_{A}\right)\right) \oplus \operatorname{Ker}\left(\mathcal{L}_{\mathfrak{g}}\left(p_{A}\right)\right)$, a Lie algebra splitting where the symbol " $\bigoplus$ " denotes the (internal) semi-direct sum of $\operatorname{Im}\left(\mathcal{L}_{\mathfrak{g}}\left(u_{A}\right)\right)$ with $\operatorname{Ker}\left(\mathcal{L}_{\mathfrak{g}}\left(p_{A}\right)\right)$. Now, definitions give $\operatorname{Im}\left(\mathcal{L}_{\mathfrak{g}}\left(u_{A}\right)\right) \cong$ $\mathcal{L}_{\mathfrak{g}}(\mathbb{K}):=\left(\mathbb{K} \otimes_{\mathbb{K}} \mathfrak{g}\right)_{\mathbf{0}}=\mathfrak{g}_{\mathbf{0}}$, and we fix the simpler notation $\mathfrak{n}_{\mathfrak{g}}(A):=\operatorname{Ker}\left(\mathcal{L}_{\mathfrak{g}}\left(p_{A}\right)\right)$. Then

$$
\mathcal{L}_{\mathfrak{g}}(A)=\mathfrak{g}_{\mathbf{0}} \oplus \mathfrak{n}_{\mathfrak{g}}(A) \quad \forall A \in(\mathbf{W} \text { salg })_{\mathbb{K}}
$$

In the following, we shall refer to $(2.1)$ as to "pointwise splitting for $\mathcal{L}_{\mathfrak{g}}$ " — or simply "for $\mathfrak{g}$ " itself.

Now note that $A=A_{\mathbf{0}} \oplus A_{\mathbf{1}}$ with $A_{\mathbf{0}}=\mathbb{K} \oplus \AA_{\mathbf{0}}$ and $A_{\mathbf{1}}=\AA_{1}$; therefore

$$
\mathcal{L}_{\mathfrak{g}}(A):=\left(A \otimes_{\mathbb{K}} \mathfrak{g}\right)_{\mathbf{0}}=\left(A_{\mathbf{0}} \otimes_{\mathbb{K}} \mathfrak{g}_{\mathbf{0}}\right) \oplus\left(A_{\mathbf{1}} \otimes_{\mathbb{K}} \mathfrak{g}_{\mathbf{1}}\right)=\mathfrak{g}_{\mathbf{0}} \oplus\left(\stackrel{\circ}{\mathbf{0}}_{\mathbf{K}} \otimes_{\mathbb{K}} \mathfrak{g}_{\mathbf{0}}\right) \oplus\left(A_{\mathbf{1}} \otimes_{\mathbb{K}} \mathfrak{g}_{\mathbf{1}}\right)
$$

from which it clearly follows that $\mathfrak{n}_{\mathfrak{g}}(A):=\operatorname{Ker}\left(\mathcal{L}_{\mathfrak{g}}\left(p_{A}\right)\right)=\left(\AA_{\mathbf{0}} \otimes_{\mathbb{K}} \mathfrak{g}_{\mathbf{0}}\right) \oplus\left(\AA_{\mathbf{1}} \otimes_{\mathbb{K}} \mathfrak{g}_{\mathbf{1}}\right)$ for all $A \in(\mathbf{W s a l g})_{\mathbb{K}}$. This in turn entails that the Lie algebra $\mathfrak{n}_{\mathfrak{g}}(A)$ is nilpotent.

2.3.3. The Lie subgroup $N_{G}(A)$. Let again $G$ be a Lie supergroup over $\mathbb{K}$, and $\mathfrak{g}:=\operatorname{Lie}(G)$ be its tangent Lie superalgebra. For any $A \in(\mathbf{W} \text { salg })_{\mathbb{K}}$, the Lie group $G(A)$ and the Lie algebra $\mathfrak{g}(A):=\mathcal{L}_{\mathfrak{g}}(A)-$ also equal to $\operatorname{Lie}(G)(A)=\operatorname{Lie}(G(A))$, cf. $\S 2.2 .2$ — are linked by the exponential map $\exp : \mathfrak{g}(A) \longrightarrow G(A)$ which is a local isomorphism (either in the smooth, analytic or holomorphic sense, as usual). Similarly for the "even counterparts" we have also the local isomorphism $\exp _{\mathbf{0}}: \mathfrak{g}_{\mathbf{0}} \longrightarrow G_{\mathbf{0}}(\mathbb{K})$ with $\exp \mathbf{0}_{\mathbf{0}}=\left.\exp \right|_{\mathfrak{g}_{\mathbf{0}}}$ if we think at $\mathfrak{g}_{0}$ as embedded into $\mathfrak{g}(A):=\mathcal{L}_{\mathfrak{g}}(A)=\mathfrak{g}_{\mathbf{0}} \oplus \mathfrak{n}_{\mathfrak{g}}(A)-\operatorname{cf}$. (2.1).

Now, since the Lie algebra $\mathfrak{n}_{\mathfrak{g}}(A)$ is nilpotent - cf. $\S 2.3 .2$ - its image $\exp \left(\mathfrak{n}_{\mathfrak{g}}(A)\right)$ for the exponential map is a (closed, connected) nilpotent Lie subgroup of $G(A)$. Furthermore, let us use notation $\mathfrak{g}\left(p_{A}\right):=$ $\mathcal{L}_{\mathfrak{g}}\left(p_{A}\right)$ and $\mathfrak{g}\left(u_{A}\right):=\mathcal{L}_{\mathfrak{g}}\left(u_{A}\right)$, and consider the diagram

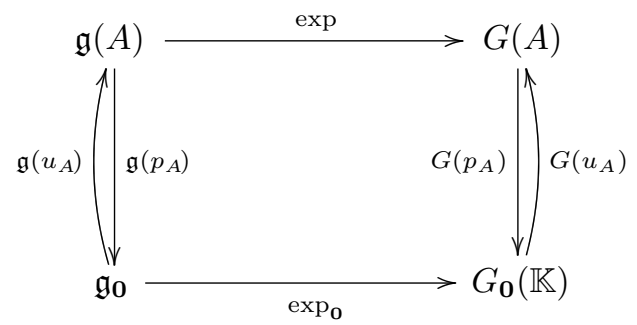

This diagram is commutative, hence in particular $G\left(p_{A}\right) \circ \exp =\exp _{\mathbf{0}} \circ \mathfrak{g}\left(p_{A}\right)$, which in turn implies at once $G\left(p_{A}\right)\left(\exp \left(\mathfrak{n}_{\mathfrak{g}}(A)\right)\right)=\exp _{\mathbf{0}}\left(\mathfrak{g}\left(p_{A}\right)\left(\mathfrak{n}_{\mathfrak{g}}(A)\right)\right)=\exp _{\mathbf{0}}\left(\left\{0_{\mathfrak{g}_{\mathbf{0}}}\right\}\right)=\left\{1_{G_{\mathbf{0}}(\mathbb{K})}\right\}$ because $\mathfrak{n}_{\mathfrak{g}}(A):=\operatorname{Ker}\left(\mathfrak{g}\left(p_{A}\right)\right) ;$ so in the end $\exp \left(\mathfrak{n}_{\mathfrak{g}}(A)\right) \subseteq \operatorname{Ker}\left(G\left(p_{A}\right)\right)=: N_{G}(A)$.

The fact that $\exp : \mathfrak{g}(A) \longrightarrow G(A)$ is a local isomorphism, together with pointwise splittings namely, $\mathfrak{g}(A)=\mathfrak{g}_{\mathbf{0}} \oplus \mathfrak{n}_{\mathfrak{g}}(A)$ and $G(A)=G_{\mathbf{0}}(\mathbb{K}) \ltimes N_{G}(A)$ - and $\operatorname{dim}\left(\mathfrak{g}_{\mathbf{0}}\right)=\operatorname{dim}\left(G_{\mathbf{0}}(\mathbb{K})\right)$, jointly imply 
$\operatorname{dim}\left(\mathfrak{n}_{\mathfrak{g}}(A)\right)=\operatorname{dim}\left(N_{G}(A)\right)$. On the other hand, as $\mathfrak{n}_{\mathfrak{g}}(A)$ is nilpotent, its exponential map - i.e., just the restriction to $\mathfrak{n}_{\mathfrak{g}}(A)$ of exp : $\mathfrak{g}(A) \longrightarrow G(A)$ — is actually a global isomorphism of $\mathbb{K}$-manifolds from $\mathfrak{n}_{\mathfrak{g}}(A)$ to $\exp \left(\mathfrak{n}_{\mathfrak{g}}(A)\right)$. It then follows that $\operatorname{dim}\left(\exp \left(\mathfrak{n}_{\mathfrak{g}}(A)\right)\right)=\operatorname{dim}\left(N_{G}(A)\right)$. Letting $N_{G}(A)^{\circ}$ be the connected component of $N_{G}(A)$, all the above eventually gives $\exp \left(\mathfrak{n}_{\mathfrak{g}}(A)\right)=N_{G}(A)^{\circ}$.

We shall now analyze $\exp \left(\mathfrak{n}_{\mathfrak{g}}(A)\right)=N_{G}(A)^{\circ}$, eventually proving that it coincides with $N_{G}(A)$.

We denote again by $G$ the Weil-Berezin functor of $\mathcal{A}$-points (cf. §2.1.3) of our Lie supergroup $G$. In detail, for $A \in(\mathbf{W s a l g})_{\mathbb{K}}$ the group $G(A)$ of $A$-points of $G$ is defined as $G(A):=G_{A}=\bigsqcup_{g \in|G|} G_{A, g}$ where $|G|$ is the underlying topological space of $G$ and $G_{A, g}:=\operatorname{Hom}_{(\mathbf{s a l g})_{\mathrm{K}}}\left(\mathcal{O}_{G, g}, A\right)$, with $\mathcal{O}_{G, g}$ being the stalk of the structure sheaf of $G$ (now thought of as a locally ringed superspace) at the point $g \in|G|$. We adopt the canonical identification $|G|=G(\mathbb{K})$ via $g \mapsto e v_{g}$ with $e v_{g}: \mathcal{O}_{G, g} \longrightarrow \mathbb{K}$ given by $f \mapsto e v_{g}(f):=f(g)$.

For every $g_{A} \in G_{A, g}$ we have $\widetilde{g}_{A}:=p_{A} \circ g_{A}$ (cf. §2.1.1) which coincides with $e v_{g}$; moreover, the very definition gives also $\widetilde{g}_{A}:=p_{A} \circ g_{A}=G\left(p_{A}\right)\left(g_{A}\right)$. Finally, due to the splitting $A=\mathbb{K} \oplus \AA$, for every $g_{A} \in G(A)$, say $g_{A} \in G_{A, g}$, there exists also a unique map $\hat{g}_{A}: \mathcal{O}_{G, g} \longrightarrow \AA$ such that $g_{A}=\widetilde{g}_{A}+\hat{g}_{A}$.

Now assume $g_{A} \in N_{G}(A):=\operatorname{Ker}\left(G\left(p_{A}\right)\right)$. Then $G\left(p_{A}\right)\left(g_{A}\right)=1_{G_{A}} \in G(A)$; therefore - by the previous analysis - we have $\widetilde{g}_{A}=1$, whence $g_{A}=1+\widehat{g}_{A}$ - which can be read as the sum, in the natural sense, of maps from $\mathcal{O}_{G, 1}$ to $A$. Now, we can re-write our $g_{A}$ as $g_{A}=1+\hat{g}_{A}=\exp \left(X_{g_{A}}\right)$ with $X_{g_{A}}:=\log \left(g_{A}\right)=\sum_{n=1}^{+\infty}(-1)^{n+1} \frac{\widehat{g}_{A}^{n}}{n}$ where $\exp \left(X_{g_{A}}\right):=\sum_{n=0}^{+\infty} X_{g_{A}}^{n} / n$ ! and all powers in these formulas are given by $X_{g_{A}}^{n}(f):=\left(X_{g_{A}}(f)\right)^{n}, \widehat{g}_{A}^{n}(f):=\left(\widehat{g}_{A}(f)\right)^{n}$, etc. All this makes sense because $\operatorname{Im}\left(\widehat{g}_{A}\right) \in \AA$, thus $\widehat{g}_{A}$ is nilpotent, hence $X_{g_{A}}$ is given by a finite sum and it is nilpotent, so $\exp \left(X_{g_{A}}\right)$ is a finite sum too.

By formal properties of exponential and logarithm, since $g_{A}: \mathcal{O}_{G, 1} \longrightarrow A$ is a (superalgebra) morphism it follows from the above expansion of $g_{A}$ that $X_{g_{A}}: \mathcal{O}_{G, 1} \longrightarrow A$ is in turn a derivation; therefore cf. $\S 2.2 .2-X_{g_{A}} \in \operatorname{Lie}(G(A))=(\operatorname{Lie}(G))(A)=\mathcal{L}_{\mathfrak{g}}(A)=: \mathfrak{g}(A)$. Finally, by construction we have also $\operatorname{Im}\left(X_{g_{A}}\right) \in \stackrel{\circ}{A}$. Along with pointwise splitting $\mathfrak{g}(A)=\mathfrak{g}_{\mathfrak{0}} \oplus \mathfrak{n}_{\mathfrak{g}}(A)$ - see $(2.1)$ for $\mathfrak{g}(A):=\mathcal{L}_{\mathfrak{g}}(A)$ - and with $\mathfrak{n}_{\mathfrak{g}}(A)=\left(\AA_{\mathbf{0}} \otimes_{\mathbb{K}} \mathfrak{g}_{\mathbf{0}}\right) \oplus\left(\AA_{\mathbf{1}} \otimes_{\mathbb{K}} \mathfrak{g}_{1}\right)$ all this together eventually gives $X_{g_{A}} \in \mathfrak{n}_{\mathfrak{g}}(A)$. All in all, we get

Proposition 2.3.4. For any Lie supergroup $G$ and $A \in(\mathbf{W} \text { salg })_{\mathbb{K}}$ we have $N_{G}(A)=\exp \left(\mathfrak{n}_{\mathfrak{g}}(A)\right)$. In particular, $N_{G}(A)$ is connected nilpotent, and (globally) isomorphic, as a manifold, to $\mathfrak{n}_{\mathfrak{g}}(A)$.

Proof. Our analysis above shows that each $g_{A} \in N_{G}(A)$ can be realized as $g_{A}=\exp \left(X_{g_{A}}\right)$ with $X_{g_{A}} \in$ $\mathfrak{n}_{\mathfrak{g}}(A)$; hence $N_{G}(A) \subseteq \exp \left(\mathfrak{n}_{\mathfrak{g}}(A)\right)$; conversely, $\S 2.3 .3$ yields $\exp \left(\mathfrak{n}_{\mathfrak{g}}(A)\right)=N_{G}(A)^{\circ} \subseteq N_{G}(A)$. Thus $N_{G}(A)=\exp \left(\mathfrak{n}_{\mathfrak{g}}(A)\right)$ as claimed. The last part of the claim then is clear. See also [9] for another proof.

\subsection{Global splittings for Lie supergroups}

We present now two remarkable splittings for the groups $G(A)$ of " $A$-points" of a Lie supergroup $G$; these are natural in $A$, hence give "global splittings" of $G$ as a functor. Such a result is often stated as a splitting of the structure sheaf (e.g., in [4], [23], [25]), we provide instead a more geometrical proof.

2.4.1. Structure theorem and global splittings for Lie supergroups. Let $G$ be a Lie supergroup over $\mathbb{K}$, whose tangent Lie superalgebra is $\mathfrak{g}:=\operatorname{Lie}(G)$, and let $A \in(\mathbf{W} \text { salg })_{\mathbb{K}}$. The powers $\AA^{d}$ of the nilradical $\AA$ of $A$ form a descending sequence such that $\AA^{N}=0$ for $N \gg 0$ (cf. $§ 2.1 .1$ ). Then we consider

$$
\mathfrak{n}_{\mathfrak{g}}^{(d)}(A):=\left(\AA^{d} \otimes_{\mathbb{K}} \mathfrak{g}\right)_{\mathbf{0}}=\left(\left(\stackrel{\circ}{A}^{d}\right)_{\mathbf{0}} \otimes_{\mathbb{K}} \mathfrak{g}_{\mathbf{0}}\right) \oplus\left(\left(\stackrel{\circ}{ }^{d}\right)_{\mathbf{1}} \otimes_{\mathbb{K}} \mathfrak{g}_{1}\right) \quad \forall d \in \mathbb{N}_{+}
$$

this in turn yields a decreasing filtration of Lie subalgebras of $\mathfrak{n}_{\mathfrak{g}}(A)$, with $\mathfrak{n}_{\mathfrak{g}}^{(N)}(A)=0$ for $N \gg 0$.

Consider the case of an element $\eta Y:=\eta \otimes Y \in \mathfrak{n}_{\mathfrak{g}}(A)$ with $\eta \in A_{\mathbf{1}}, Y \in \mathfrak{g}_{\mathbf{1}}$. As $\eta^{2}=0$, if we express $\exp (\eta Y)$ as a formal series we actually have $\exp (\eta Y)=1+\eta Y$. Similarly, for every $c X=c \otimes X \in \mathfrak{n}_{\mathfrak{g}}(A)$ with $c \in A_{\mathbf{0}}, X \in \mathfrak{g}_{\mathbf{0}}$ if $c^{2}=0$ then also the formal series expression of $\exp (c X)$ reads $\exp (c X)=1+c X$. 
For later use, we fix a $\mathbb{K}$-basis $B$ of $\mathfrak{g}$ of the form $B:=B_{\mathbf{0}} \sqcup B_{\mathbf{1}}$ with $B_{\mathbf{0}}=\left\{X_{j}\right\}_{j \in J}$, resp. $B_{\mathbf{1}}=\left\{Y_{i}\right\}_{i \in I}$, being a $\mathbb{K}$-basis of $\mathfrak{g}_{\mathbf{0}}$, resp. of $\mathfrak{g}_{1}$. Moreover, we fix any total order $\leq$ on $B$ - or, equivalently, on $I \sqcup J$ - such that all elements from $B_{\mathbf{0}}$ are less than those of $B_{1}$ - in a nutshell, $B_{\mathbf{0}} \leq B_{\mathbf{1}}$.

Now consider the $\mathbb{K}$-algebra $\mathbb{K}\left\langle\left\langle Z_{1}, Z_{2}\right\rangle\right\rangle$ of formal power series in the non-commutative variables $Z_{1}$ and $Z_{2}$. The Campbell-Baker-Hausdorff formula in $\mathbb{K}\left\langle\left\langle Z_{1}, Z_{2}\right\rangle\right\rangle$ is $\exp \left(Z_{1}\right) \cdot \exp \left(Z_{2}\right)=\exp \left(Z_{1} * Z_{2}\right)$ with $Z_{1} * Z_{2}:=\log \left(\exp \left(Z_{1}\right) \cdot \exp \left(Z_{2}\right)\right) \in \mathbb{K}\left\langle\left\langle Z_{1}, Z_{2}\right\rangle\right\rangle$. More precisely, the formal series expansion of $Z_{1} * Z_{2}$ can be re-arranged as a formal series $Z_{1} * Z_{2}=\sum_{n=1}^{+\infty} L_{n}\left(Z_{1}, Z_{2}\right)$ where each $L_{n}\left(Z_{1}, Z_{2}\right)$ is a homogeneous Lie monomial of degree $n$ in the free Lie $\mathbb{K}$-algebra $\left\langle Z_{1}, Z_{2}\right\rangle_{L i e}^{\mathbb{K}}$ generated by $Z_{1}$ and $Z_{2}$. In particular, if one replaces $Z_{1}$ and $Z_{2}$ with elements $z_{1}$ and $z_{2}$ sitting in some nilpotent Lie algebra, then all but finitely many of the $L_{n}\left(z_{1}, z_{2}\right)$ 's do vanish, hence $z_{1} * z_{2}$ can be written as a finite sum.

Our next goal is another description of $N_{G}(A)=\exp \left(\mathfrak{n}_{\mathfrak{g}}(A)\right)$. We need an auxiliary result:

Lemma 2.4.2. Let $S_{1}, \ldots, S_{\ell} \in \mathfrak{n}_{\mathfrak{g}}(A)$ with $S_{i} \in \mathfrak{n}_{\mathfrak{g}}^{\left(d_{i}\right)}(A), d_{i} \in \mathbb{N}_{+} \quad(i=1, \ldots, \ell)$. Then there exist $T_{1}, \ldots, T_{k} \in \mathfrak{n}_{\mathfrak{g}}(A)$ such that $T_{j} \in \mathfrak{n}_{\mathfrak{g}}^{\left(\partial_{j}\right)}(A)$ with $\partial_{j} \geqslant d_{a_{j}}+d_{b_{j}}$ and $a_{j}, b_{j} \in\{1, \ldots, \ell\} \quad(j=1, \ldots, k)$, and

$$
\exp \left(S_{1}+\cdots+S_{\ell}\right)=\exp \left(S_{1}\right) \cdots \exp \left(S_{\ell}\right) \exp \left(T_{1}\right) \cdots \exp \left(T_{k}\right)
$$

Proof. Writing all exponentials as formal series (actually finite sums!) the claim follows at once from definitions by induction on $\ell$ via a straightforward application of Baker-Campbell-Hausdorff formula.

We can now provide our new description of the subgroup $N_{G}(A)=\exp \left(\mathfrak{n}_{\mathfrak{g}}(A)\right)$ :

Proposition 2.4.3. The subgroup $N_{G}(A)=\exp \left(\mathfrak{n}_{\mathfrak{g}}(A)\right)$ of $G(A)$ is generated by the set

$$
\Gamma_{B}:=\left\{\exp \left(t_{j} X_{j}\right), \exp \left(\eta_{i} Y_{i}\right) \mid t_{j} \in \stackrel{\circ}{A}_{\mathbf{0}}, \eta_{i} \in \stackrel{\circ}{A}_{\mathbf{1}}=A_{\mathbf{1}}, \forall j \in J, i \in I\right\}
$$

where $\left\{X_{j}\right\}_{j \in J} \sqcup\left\{Y_{i}\right\}_{i \in I}=B_{\mathbf{0}} \sqcup B_{\mathbf{1}}=B$ is the $\mathbb{K}$-basis of $\mathfrak{g}$ chosen in \$2.4.1 above.

Proof. Let $n \in N_{G}(A)=\exp \left(\mathfrak{n}_{\mathfrak{g}}(A)\right)$, say $n=\exp (Z)$ with $Z \in \mathfrak{n}_{\mathfrak{g}}(A)$; clearly we can assume $Z \neq 0$. Using our fixed, ordered, $\mathbb{K}$-basis $B$ of $\mathfrak{g}$ our $Z$ expands into $Z=\sum_{j \in J} t_{j}^{\prime} X_{j}+\sum_{i \in I} \eta_{i}^{\prime} Y_{i}$ for some $t_{j}^{\prime} \in \AA_{\mathbf{0}}$ and $\eta_{i}^{\prime} \in \AA_{\mathbf{1}}$, by the very definition of $\mathfrak{n}_{\mathfrak{g}}(A)$. By Lemma 2.4.2, this implies that

$$
\exp (Z)=\exp \left(\sum_{j \in J} t_{j}^{\prime} X_{j}+\sum_{i \in I} \eta_{i}^{\prime} Y_{i}\right)=\overrightarrow{\prod_{j \in J}} \exp \left(t_{j}^{\prime} X_{j}\right) \overrightarrow{\prod_{i \in I}} \exp \left(\eta_{i}^{\prime} Y_{i}\right) \cdot \exp \left(Z_{1}^{(1)}\right) \cdots \exp \left(Z_{k_{1}}^{(1)}\right)
$$

for some $Z_{1}^{(1)}, \ldots, Z_{k_{1}}^{(1)} \in \mathfrak{n}_{\mathfrak{g}}(A)$, where $\vec{\prod}_{j \in J}$ and $\vec{\prod}_{i \in I}$ denotes ordered products. Even more, the Lemma ensures that these $Z_{h}^{(1)}$ 's "lie deeper", in the decreasing filtration of $\mathfrak{n}_{\mathfrak{g}}(A)$ given by the $\mathfrak{n}_{\mathfrak{g}}^{(d)}(A)$ 's, than the initial $Z$ we started with: so we can iterate this argument and finally stop after finitely many steps.

2.4.4. Special exponentials in $G(A)$. Before going on, let us consider elements in $G(A)$ of the form $\exp (t X)$ or $\exp (\eta Y)$ - with $t \in A_{\mathbf{0}}$ such that $t^{2}=0, \eta \in \AA_{1}, X \in \mathfrak{g}_{\mathbf{0}}, Y \in \mathfrak{g}_{\mathbf{1}}$. Since both $t$ and $\eta$ have square zero, the formal power series expansion of both $\exp (t X)$ and $\exp (\eta Y)$ stops at first order, i.e. it reads $\exp (t X)=(1+t X)$ and $\exp (\eta Y)=(1+\eta Y)$ respectively. More in general, we consider elements of the form $\exp (\mathcal{X}), \exp (\mathcal{Y}) \in \exp \left(\mathfrak{n}_{\mathfrak{g}}(A)\right)=N_{G}(A)$ with $\mathcal{X} \in \AA_{0} \otimes \mathfrak{g}_{\mathbf{0}}$ and $\mathcal{Y} \in \AA_{\mathbf{1}} \otimes \mathfrak{g}_{\mathbf{1}}=A_{\mathbf{1}} \otimes \mathfrak{g}_{\mathbf{1}}$. As $\AA_{\mathbf{0}}$ and $A_{1}$ are nilpotent, the power series expansion of $\exp (\mathcal{X})$ and $\exp (\mathcal{Y})$ can be seen again as polynomials.

In the next Lemma we collect some identities in $G(A)$ involving these "special exponentials".

Lemma 2.4.5. Let $A \in(\mathbf{W s a l g})_{\mathbb{K}}, \eta, \eta^{\prime}, \eta^{\prime \prime} \in A_{\mathbf{1}}, \eta_{i} \in A_{\mathbf{1}}$ (for all $\left.i \in I\right), Y, Y^{\prime} \in \mathfrak{g}_{\mathbf{1}}, X \in \mathfrak{g}_{\mathbf{0}}$ and $g_{0} \in G_{\mathbf{0}}(A)$. Then inside $G(A)$ we have

(a) $\quad\left(1+\eta \eta^{\prime}\left[Y, Y^{\prime}\right]\right)=\exp \left(\eta \eta^{\prime}\left[Y, Y^{\prime}\right]\right) \in G_{\mathbf{0}}(A)$

(b) $(1+\eta Y) g_{0}=g_{0}\left(1+\eta A d\left(g_{0}^{-1}\right)(Y)\right), \quad \exp \left(\sum_{i \in I} \eta_{i} Y_{i}\right) g_{0}=g_{0} \exp \left(\sum_{i \in I} \eta_{i} A d\left(g_{0}^{-1}\right)\left(Y_{i}\right)\right)$ 


$$
\left(1+\eta^{\prime} Y^{\prime}\right)\left(1+\eta^{\prime \prime} Y^{\prime \prime}\right)=\left(1+\eta^{\prime \prime} \eta^{\prime}\left[Y^{\prime}, Y^{\prime \prime}\right]\right)\left(1+\eta^{\prime \prime} Y^{\prime \prime}\right)\left(1+\eta^{\prime} Y^{\prime}\right)
$$

$$
\left(1+\eta Y^{\prime}\right)\left(1+\eta Y^{\prime \prime}\right)=\left(1+\eta\left(Y^{\prime}+Y^{\prime \prime}\right)\right)=\left(1+\eta Y^{\prime \prime}\right)\left(1+\eta Y^{\prime}\right)
$$

$$
\begin{aligned}
\left(1+\eta^{\prime} Y\right)\left(1+\eta^{\prime \prime} Y\right) & =\left(1+\eta^{\prime \prime} \eta^{\prime} Y^{\langle 2\rangle}\right)\left(1+\left(\eta^{\prime}+\eta^{\prime \prime}\right) Y\right) \\
(1+\eta Y)\left(1+\eta^{\prime} \eta^{\prime \prime} X\right) & =\left(1+\eta^{\prime} \eta^{\prime \prime} X\right)\left(1+\eta \eta^{\prime} \eta^{\prime \prime}[Y, X]\right)(1+\eta Y)= \\
= & \left(1+\eta^{\prime} \eta^{\prime \prime} X\right)(1+\eta Y)\left(1+\eta \eta^{\prime} \eta^{\prime \prime}[Y, X]\right)
\end{aligned}
$$

(g) Let $(h, k):=h k h^{-1} k^{-1}$ be the commutator of elements $h$ and $k$ in a group. Then

$$
\begin{gathered}
\left((1+\eta Y),\left(1+\eta^{\prime} Y^{\prime}\right)\right)=\left(1+\eta^{\prime} \eta\left[Y, Y^{\prime}\right]\right),\left((1+\eta Y),\left(1+\eta Y^{\prime}\right)\right)=\left(1+\eta\left(Y+Y^{\prime}\right)\right) \\
\left(\left(1+\eta^{\prime} Y\right),\left(1+\eta^{\prime \prime} Y\right)\right)=\left(1+\eta^{\prime \prime} \eta^{\prime} Y^{<2\rangle}\right)^{2}=\left(1+\eta^{\prime \prime} \eta^{\prime} 2 Y^{<2\rangle}\right)=\left(1+\eta^{\prime \prime} \eta^{\prime}[Y, Y]\right)
\end{gathered}
$$

(N.B.: taking the rightmost term in the last identity, the latter is a special case of the first).

(h) For any $n \in \mathbb{N}_{+}$, there exist unique $T_{\mathbf{0}}^{(n)}, T_{\mathbf{1}}^{(n)} \in\left\langle Z_{1}, Z_{2}\right\rangle_{\text {Lie }}^{\mathbb{K}}$, independent of $A$, such that:

- $T_{\mathbf{0}}^{(n)}$ is a $\mathbb{K}$-linear combination of Lie monomials of even degree greater than $n$,

- $T_{1}^{(n)}$ is a $\mathbb{K}$-linear combination of Lie monomials of odd degree greater than $n$,

- setting $d_{1}:=\operatorname{dim}\left(\mathfrak{g}_{1}\right)$, for any $\mathcal{Y}^{\prime}, \mathcal{Y}^{\prime \prime} \in A_{1} \bigotimes_{\mathbb{K}} \mathfrak{g}_{1}$ we have

$$
\exp \left(\mathcal{Y}^{\prime}\right) \exp \left(\mathcal{Y}^{\prime \prime}\right)=\exp \left(P_{\mathbf{0}}^{\left(d_{1}\right)}\left(\mathcal{Y}^{\prime}, \mathcal{Y}^{\prime \prime}\right)\right) \exp \left(\mathcal{Y}^{\prime}+\mathcal{Y}^{\prime \prime}+P_{\mathbf{1}}^{\left(d_{1}\right)}\left(\mathcal{Y}^{\prime}, \mathcal{Y}^{\prime \prime}\right)\right)
$$

with $P_{\mathbf{0}}^{\left(d_{1}\right)}:=T_{\mathbf{0}}^{(1)} * T_{\mathbf{0}}^{(2)} * \cdots * T_{\mathbf{0}}^{\left(d_{1}-1\right)}$ and $P_{\mathbf{1}}^{\left(d_{1}\right)}:=T_{\mathbf{1}}^{\left(d_{1}-1\right)}+\cdots+T_{\mathbf{1}}^{(2)}+T_{\mathbf{1}}^{(1)}$.

Proof. Writing all exponentials as formal power series (actually finite sum, as noticed above), claims (a) through $(g)$ follow at once from definitions, via straightforward applications of the Baker-Campbell-Hausdorff formula. Claim (a) is even simpler, since $\left(1+\eta \eta^{\prime}\left[Y, Y^{\prime}\right]\right)$ is just the formal power series expansion of $\exp \left(\eta \eta^{\prime}\left[Y, Y^{\prime}\right]\right)$, and the latter belong to $\exp \left(\stackrel{\circ}{A}_{\mathbf{0}} \mathfrak{g}_{\mathbf{0}}\right) \subseteq G_{\mathbf{0}}(A)$.

Claim $(h)$ requires some more work. An equivalent formulation of it is that the identity

$$
\mathcal{Y}^{\prime} * \mathcal{Y}^{\prime \prime}=P_{\mathbf{0}}^{\left(d_{1}\right)}\left(\mathcal{Y}^{\prime}, \mathcal{Y}^{\prime \prime}\right) *\left(\mathcal{Y}^{\prime}+\mathcal{Y}^{\prime \prime}+P_{\mathbf{1}}^{\left(d_{1}\right)}\left(\mathcal{Y}^{\prime}, \mathcal{Y}^{\prime \prime}\right)\right)
$$

holds true for some uniquely determined Lie polynomials $P_{\mathbf{0}}^{\left(d_{1}\right)}:=T_{\mathbf{0}}^{(1)} * T_{\mathbf{0}}^{(2)} * \cdots * T_{\mathbf{0}}^{\left(d_{1}-1\right)}$ and $P_{\mathbf{1}}^{\left(d_{1}\right)}:=$ $T_{\mathbf{1}}^{\left(d_{1}-1\right)}+\cdots+T_{\mathbf{1}}^{(2)}+T_{\mathbf{1}}^{(1)}$ with the $T_{\mathbf{0} / \mathbf{1}}^{(i)}$ s having the properties mentioned above.

We start working with the product “*” in $\left\langle Z_{1}, Z_{2}\right\rangle_{L i e}^{\mathbb{K}}$. As a matter of terminology, we call order of any non-zero Lie polynomial $P$ in two variables the least degree of a homogeneous monomial occurring with non-zero coefficient in the standard $\mathbb{K}$-linear expansion of $P$ (while the zero polynomial has order $-\infty$ ).

First we need some technical results. For formal $F, G$ there exist unique $R, S \in\langle F, G\rangle_{L i e}^{\mathbb{K}}$ such that

$$
F * G=R *(F+G) \quad, \quad F+G=F * S * G
$$

and $R, S$ are Lie polynomials (in $F$ and $G$ ) of order greater than 1: in fact, $R$ is the unique solution of the equation $\exp (F) \exp (G)=\exp (R) \exp (F+G)$, while $S$ is the unique solution of the equation $\exp (F+G)=\exp (F) \exp (S) \exp (G)$, Then the explicit expression of the product "*" implies that both $R$ and $S$ have order greater than 1, as claimed; moreover, both are independent of $A$ and $\mathfrak{g}$ whatsoever. Finally, for any Lie polynomial $T$ in two variables there exist unique $T_{\mathbf{0}}$ and $T_{\mathbf{1}}$ such that $T=T_{\mathbf{0}}+T_{\mathbf{1}}$ where $T_{\mathbf{0}}$, resp. $T_{\mathbf{1}}$, is a $\mathbb{K}$-linear combination of Lie monomials of even, resp. odd, degree.

With repeated applications of (2.3) and of the identity $T=T_{\mathbf{0}}+T_{\mathbf{1}}$ we eventually find the expression $Z_{1} * Z_{2}=T_{\mathbf{0}}^{(1)} * T_{\mathbf{0}}^{(2)} * \cdots * T_{\mathbf{0}}^{(n-1)} * T^{(n)} *\left(T_{\mathbf{1}}^{(n-1)}+\cdots+T_{\mathbf{1}}^{(2)}+T_{\mathbf{1}}^{(1)}+Z_{1}+Z_{2}\right)$ for arbitrarily big $n \in \mathbb{N}$, where each Lie polynomial $T_{s}$ has order greater than $s$. Finally, we can re-write this last formula as

$$
Z_{1} * Z_{2}=P_{\mathbf{0}}^{(n)}\left(Z_{1}, Z_{2}\right) * T^{(n)} *\left(Z_{1}+Z_{2}+P_{\mathbf{1}}^{(n)}\left(Z_{1}, Z_{2}\right)\right)
$$

for all $n \in \mathbb{N}$, with $P_{\mathbf{0}}^{(n)}:=T_{\mathbf{0}}^{(1)} * T_{\mathbf{0}}^{(2)} * \cdots * T_{\mathbf{0}}^{(n-1)}$ and $P_{\mathbf{1}}^{(n)}:=T_{\mathbf{1}}^{(n-1)}+\cdots+T_{\mathbf{1}}^{(2)}+T_{\mathbf{1}}^{(1)}$.

But every Lie monomial of degree $m>d_{1}:=\operatorname{dim}\left(\mathfrak{g}_{1}\right)$ vanishes on $A_{1} \otimes_{\mathbb{K}} \mathfrak{g}_{1}$, hence $T^{(m-1)}$ vanishes too. It follows that for $n=d_{1}$ replacing $\mathcal{Y}^{\prime}$ for $Z_{1}$ and $\mathcal{Y}^{\prime \prime}$ for $Z_{2}$ in (2.4) we eventually get (2.2), q.e.d. 
We still need to introduce some auxiliary objects associated with $G$ :

Definition 2.4.6. Let $G$ be a Lie supergroup, as above. For any $A \in(\mathbf{W s a l g})_{\mathbb{K}}$, we define:

(b) $\quad \exp \left(A_{\mathbf{1}} \otimes_{\mathbb{K}} \mathfrak{g}_{\mathbf{1}}\right):=\left\{\exp (\mathcal{Y}) \mid \mathcal{Y} \in A_{\mathbf{1}} \otimes_{\mathbb{K}} \mathfrak{g}_{\mathbf{1}}\right\} \quad\left(\subseteq G^{-}(A)\right)$

(c) $\quad N_{G}^{[2]}(A):=\exp \left(A_{1}^{[2]} \otimes_{\mathbb{K}}\left[\mathfrak{g}_{1}, \mathfrak{g}_{\mathbf{1}}\right]\right) \quad\left(\subseteq N_{G_{\mathbf{0}}}\left(A_{\mathbf{0}}\right)=N_{G}(A) \bigcap G_{\mathbf{0}}(A)\right)$

(d) for any fixed $\mathbb{K}$-basis $\left\{Y_{i}\right\}_{i \in I}$ of $\mathfrak{g}_{1}$ (for some index set $I$ ) and any fixed total order in $I$,

$$
G_{-}^{<}(A):=\left\{\prod_{i \in I}^{\rightarrow}\left(1+\eta_{i} Y_{i}\right) \mid \eta_{i} \in A_{\mathbf{1}} \forall i \in I\right\} \quad\left(\subseteq G^{-}(A)\right)
$$

where $\overrightarrow{\prod_{i \in I}}$ denotes an ordered product — with respect to the fixed total order in $I$.

Remark 2.4.7. By definition, $\exp \left(A_{1} \otimes_{\mathbb{K}} \mathfrak{g}_{1}\right)$ contains the set of generators of $G^{-}(A)$; therefore, the former generates a subgroup $\left\langle\exp \left(A_{\mathbf{1}} \otimes_{\mathbb{K}} \mathfrak{g}_{\mathbf{1}}\right)\right\rangle$ of $G(A)$ that contains $G^{-}(A)$. On the other hand, for any $\sum_{s=1}^{n} \eta_{s} Y_{s} \in A_{\mathbf{1}} \otimes_{\mathbb{K}} \mathfrak{g}_{\mathbf{1}}$, the formal series expansion of $\exp \left(\sum_{s=1}^{n} \eta_{s} Y_{s}\right)$ yields

$$
\exp \left(\sum_{s=1}^{n} \eta_{s} Y_{s}\right)=\prod_{\sigma \in \mathcal{S}_{n}}\left(1+\eta_{\sigma(1)} Y_{\sigma(1)} / n !\right) \cdot\left(1+\eta_{\sigma(2)} Y_{\sigma(2)} / n !\right) \cdots\left(1+\eta_{\sigma(n)} Y_{\sigma(n)} / n !\right)
$$

that implies $\left\langle\exp \left(A_{\mathbf{1}} \otimes_{\mathbb{K}} \mathfrak{g}_{\mathbf{1}}\right)\right\rangle \subseteq G^{-}(A)$. The outcome is that $G^{-}(A)=\left\langle\exp \left(A_{\mathbf{1}} \otimes_{\mathbb{K}} \mathfrak{g}_{\mathbf{1}}\right)\right\rangle$.

From now on, we fix a $\mathbb{K}$-basis $\left\{Y_{i}\right\}_{i \in I}$ of $\mathfrak{g}_{1}$ (for some index set $I$ ) and we fix in $I$ a total order, as in Definition 2.4.6(d). Our first result provides new, interesting factorizations for $G(A)$ :

Proposition 2.4.8. Let $G$ be a Lie supergroup as above, let $\left\{Y_{i}\right\}_{i \in I}$ be a totally ordered $\mathbb{K}$-basis of $\mathfrak{g}_{1}$ (for some total order in the set $I)$ and let $A \in(\mathbf{W} \mathbf{s a l g})_{\mathbb{K}}$ be any Weil superalgebra. Then:

(a) $G^{-}(A)$ coincides with the subgroup $\left\langle G_{-}^{<}(A)\right\rangle$ of $G(A)$ generated by $G_{-}^{<}(A)$ and with the subgroup $\left\langle\exp \left(A_{\mathbf{1}} \otimes_{\mathbb{K}} \mathfrak{g}_{\mathbf{1}}\right)\right\rangle$ generated by $\exp \left(A_{\mathbf{1}} \otimes_{\mathbb{K}} \mathfrak{g}_{\mathbf{1}}\right) ;$

(b) there exist set-theoretic factorizations (with respect to the group product ". ")

$$
\begin{aligned}
& G^{-}(A)=N_{G}^{[2]}(A) \cdot G_{-}^{<}(A) \quad, \quad G^{-}(A)=G_{-}^{<}(A) \cdot N_{G}^{[2]}(A) \\
& N_{G}(A)=N_{G_{\mathbf{0}}}\left(A_{\mathbf{0}}\right) \cdot G_{-}^{<}(A) \quad, \quad N_{G}(A)=G_{-}^{<}(A) \cdot N_{G_{\mathbf{0}}}\left(A_{\mathbf{0}}\right) \\
& G(A)=G_{0}(A) \cdot G_{-}^{<}(A) \quad, \quad G(A)=G_{-}^{<}(A) \cdot G_{0}(A)
\end{aligned}
$$

(c) there exist set-theoretic factorizations (with respect to the group product ".")

$$
\begin{aligned}
& G^{-}(A)=N_{G}^{[2]}(A) \cdot \exp \left(A_{\mathbf{1}} \otimes_{\mathbb{K}} \mathfrak{g}_{\mathbf{1}}\right) \quad, \quad G^{-}(A)=\exp \left(A_{\mathbf{1}} \otimes_{\mathbb{K}} \mathfrak{g}_{\mathbf{1}}\right) \cdot N_{G}^{[2]}(A) \\
& N_{G}(A)=N_{G_{\mathbf{0}}}\left(A_{\mathbf{0}}\right) \cdot \exp \left(A_{\mathbf{1}} \otimes_{\mathbb{K}} \mathfrak{g}_{\mathbf{1}}\right) \quad, \quad N_{G}(A)=\exp \left(A_{\mathbf{1}} \otimes_{\mathbb{K}} \mathfrak{g}_{\mathbf{1}}\right) \cdot N_{G_{\mathbf{0}}}\left(A_{\mathbf{0}}\right) \\
& G(A)=G_{\mathbf{0}}(A) \cdot \exp \left(A_{\mathbf{1}} \otimes_{\mathbb{K}} \mathfrak{g}_{\mathbf{1}}\right) \quad, \quad G(A)=\exp \left(A_{\mathbf{1}} \otimes_{\mathbb{K}} \mathfrak{g}_{\mathbf{1}}\right) \cdot G_{\mathbf{0}}(A)
\end{aligned}
$$

Proof. (a) Remark 2.4.7 proves that $\left\langle\exp \left(A_{\mathbf{1}} \otimes_{\mathbb{K}} \mathfrak{g}_{1}\right)\right\rangle=\left\langle G_{-}^{<}(A)\right\rangle$, so we are left to prove $\left\langle G_{-}^{<}(A)\right\rangle=$ $G^{-}(A)$. By definition, $G^{-}(A)$ is the subgroup in $G(A)$ generated by $\left\{(1+\eta Y) \mid \eta \in A_{\mathbf{1}}, Y \in \mathfrak{g}_{\mathbf{1}}\right\}$ : thus it is enough to prove that each $(1+\eta Y)$ lies in $\left\langle G_{-}^{<}(A)\right\rangle$. For $Y \in \mathfrak{g}_{1}$ let $Y=\sum_{i \in I} c_{i} Y_{i}$ be its $\mathbb{K}-$ linear expansion with respect to the basis $\left\{Y_{i}\right\}_{i \in I}$ of $\mathfrak{g}_{1}$; then repeated applications of Lemma 2.4.5 $(d)$ yield

$$
(1+\eta Y)=\left(1+\eta \sum_{i \in I} c_{i} Y_{i}\right)=\left(1+\sum_{i \in I}\left(c_{i} \eta\right) Y_{i}\right)=\vec{\prod}_{i \in I}\left(1+\left(c_{i} \eta\right) Y_{i}\right) \in G_{-}^{\leq}(A), \quad \text { q.e.d. }
$$

(b) This part can be proved much like Proposition 4.2.7 in [16], but now applying Lemma 2.4.5. 
(c) Starting with (2.8), it is enough to prove the left-hand side, i.e. $G^{-}(A)=N_{G}^{[2]}(A) \cdot \exp \left(A_{\mathbf{1}} \otimes_{\mathbb{K}} \mathfrak{g}_{\mathbf{1}}\right)$.

First of all, the inclusion $N_{G}^{[2]}(A) \cdot \exp \left(A_{\mathbf{1}} \otimes_{\mathbb{K}} \mathfrak{g}_{\mathbf{1}}\right) \subseteq G^{-}(A)$ follows at once from (2.5) together with claim (a). Moreover, again by claim (a) we have $G^{-}(A)=\left\langle\exp \left(A_{1} \otimes_{\mathbb{K}} \mathfrak{g}_{1}\right)\right\rangle$ so it is enough to prove that the product of any two generators $\exp \left(\mathcal{Y}^{\prime}\right)$ and $\exp \left(\mathcal{Y}^{\prime \prime}\right)$ of $G^{-}(A)=\left\langle\exp \left(A_{1} \otimes_{\mathbb{K}} \mathfrak{g}_{1}\right)\right\rangle$ lies in $N_{G}^{[2]}(A) \cdot \exp \left(A_{1} \otimes_{\mathbb{K}} \mathfrak{g}_{1}\right)$. In fact, this follows at once from the identity in Lemma 2.4.5 $(h)$.

The same argument used above also applies to prove (2.9).

Finally, the following chain of identities $G(A)=G_{\mathbf{0}}(\mathbb{K}) \cdot N_{G}(A)=G_{\mathbf{0}}(\mathbb{K}) \cdot N_{G_{\mathbf{0}}}\left(A_{\mathbf{0}}\right) \cdot \exp \left(A_{\mathbf{1}} \otimes_{\mathbb{K}} \mathfrak{g}_{\mathbf{1}}\right)=$ $=G_{\mathbf{0}}\left(A_{\mathbf{0}}\right) \cdot \exp \left(A_{\mathbf{1}} \otimes_{\mathbb{K}} \mathfrak{g}_{\mathbf{1}}\right)=G_{\mathbf{0}}(A) \cdot \exp \left(A_{\mathbf{1}} \otimes_{\mathbb{K}} \mathfrak{g}_{\mathbf{1}}\right)$, using the pointwise splitting $G(A) \cong G_{\mathbf{0}}(\mathbb{K}) \ltimes N_{G}(A)$ — cf. $\S 2.3 .1$ - and $(2.9)$, proves $(2.10)$.

We aim to improve the previous result. We still need a technical result:

Lemma 2.4.9. Given a Lie supergroup $G$ and $A \in(\mathbf{W} \mathbf{s a l g})_{\mathbb{k}}$, let $\zeta_{i} \in A_{\mathbf{1}}(i \in I)$. Then:

(a) if $g:=\overrightarrow{\prod_{i \in I}}\left(1+\zeta_{i} Y_{i}\right) \in G_{\mathbf{0}}(A) \cap G_{-}^{<}(A)$, then $\zeta_{i}=0$ for all $i \in I$;

(b) if $g:=\exp \left(\sum_{i \in I} \zeta_{i} Y_{i}\right) \in G_{\mathbf{0}}(A) \bigcap \exp \left(A_{\mathbf{1}} \otimes_{\mathbb{K}} \mathfrak{g}_{\mathbf{1}}\right)$, then $\zeta_{i}=0$ for all $i \in I$.

Proof. (a) Recall (cf. §2.3.3) that, by definition, we have $G(A):=\coprod_{x \in|G|} \operatorname{Hom}_{(\mathbf{s a l g})_{\mathrm{K}}}\left(\mathcal{O}_{|G|, x}, A\right)$; therefore $g:=\overrightarrow{\prod_{i \in I}}\left(1+\zeta_{i} Y_{i}\right)=1+\sum_{i \in I} \zeta_{i} Y_{i}+\mathcal{O}(2)$ makes sense, as an expansion of $g$ where $\mathcal{O}(2)$ is a shorthand notation for "additional summands of higher order in the $\zeta_{i}$ 's". Let $\mathfrak{a}:=\left(\left\{\zeta_{i}\right\}_{i \in I}\right)$ be the ideal of $A$ generated by the $\zeta_{i}$ 's; then the previous expansion of $g$ yields a similar expansion $[g]_{2}:=1+\sum_{i \in I}\left[\zeta_{i}\right]_{2} Y_{i}$ inside $G\left(A / \mathfrak{a}^{2}\right):=\coprod_{x \in|G|} \operatorname{Hom}_{(\mathbf{s a l g})_{\mathbb{K}}}\left(\mathcal{O}_{|G|, x}, A / \mathfrak{a}^{2}\right)$. Moreover, from $g \in G_{\mathbf{0}}(A) \cap G_{-}^{<}(A)$ we get $[g]_{2} \in$ $G_{\mathbf{0}}\left(A / \mathfrak{a}^{2}\right) \cap G_{-}^{<}\left(A / \mathfrak{a}^{2}\right)$ too, hence - thinking of $[g]_{2}$ as an $A / \mathfrak{a}^{2}$-valued map - also $\operatorname{Im}\left([g]_{2}\right) \subseteq\left(A / \mathfrak{a}^{2}\right)_{\mathbf{0}}$.

As $\left\{Y_{i}\right\}_{i \in I}$ is a $\mathbb{K}$-basis of $\mathfrak{g}_{1}$, there exists a local system of coordinates around the unit point $1_{G} \in|G|$, say $\left\{y_{i}\right\}_{i \in I}$, such that $Y_{i}\left(y_{j}\right)=\delta_{i, j} \forall i, j \in I$. Then $[g]_{2}\left(y_{j}\right):=1+\sum_{i \in I}\left[\zeta_{i}\right]_{2} Y_{i}\left(y_{j}\right)=\left[\zeta_{j}\right]_{2}$, in particular $[g]_{2}\left(y_{j}\right)=\left[\zeta_{j}\right]_{2} \in\left(A / \mathfrak{a}^{2}\right)_{1}$; this together with $\operatorname{Im}\left([g]_{2}\right) \subseteq\left(A / \mathfrak{a}^{2}\right)_{0}$ implies $\left[\zeta_{j}\right]_{2}=[0]_{2} \in A / \mathfrak{a}^{2}$, i.e. $\zeta_{j} \in \mathfrak{a}^{2}=\left(\left\{\zeta_{i}\right\}_{i \in I}\right)^{2} \forall j \in I$ : thus $\zeta_{j} \in \mathfrak{a}^{n}$ for $n \in \mathbb{N}, j \in I$. But $\mathfrak{a}^{n}=0$ for $n \gg 0$, so $\zeta_{j}=0 \forall j \in I$, q.e.d.

(b) The same argument as in (a) applies again.

Finally, we are ready to state the main result of the present subsection. Later on, we will refer to the isomorphisms in part (a) and/or (c) of its statement as to "Global Splittings".

Theorem 2.4.10. (existence of Global Splittings for Lie supergroups)

Let $G$ be a Lie supergroup, and $\mathfrak{g}$ its tangent Lie superalgebra.

(a) The restriction of group multiplication in $G$ provides isomorphisms of (set-valued) functors

$$
\begin{aligned}
& N_{G}^{[2]} \times G_{-}^{<} \cong G^{-} \quad, \quad N_{G_{0}} \times G_{-}^{<} \cong N_{G} \quad, \quad G_{0} \times G_{-}^{<} \cong G \\
& G_{-}^{<} \times N_{G}^{[2]} \cong G^{-} \quad, \quad G_{-}^{<} \times N_{G_{0}} \cong N_{G} \quad, \quad G_{-}^{<} \times G_{0} \cong G \\
& N_{G}^{[2]} \times \exp \left((-)_{1} \otimes_{\mathbb{K}} \mathfrak{g}_{1}\right) \cong G^{-}, N_{G_{0}} \times \exp \left((-)_{1} \otimes_{\mathbb{K}} \mathfrak{g}_{\mathbf{1}}\right) \cong N_{G}, \quad G_{\mathbf{0}} \times \exp \left((-)_{\mathbf{1}} \otimes_{\mathbb{K}} \mathfrak{g}_{\mathbf{1}}\right) \cong G \\
& \exp \left((-)_{1} \otimes_{\mathbb{K}} \mathfrak{g}_{1}\right) \times N_{G}^{[2]} \cong G^{-}, \quad \exp \left((-)_{1} \otimes_{\mathbb{K}} \mathfrak{g}_{\mathbf{1}}\right) \times N_{G_{\mathbf{0}}} \cong N_{G}, \quad \exp \left((-)_{1} \otimes_{\mathbb{K}} \mathfrak{g}_{\mathbf{1}}\right) \times G_{\mathbf{0}} \cong G
\end{aligned}
$$

with $\exp \left((-)_{\mathbf{1}} \otimes_{\mathbb{K}} \mathfrak{g}_{\mathbf{1}}\right)$ the set-valued functor $(\mathbf{W} \mathbf{s a l g})_{\mathbb{K}} \longrightarrow($ sets $)$ given by $A \mapsto \exp \left(A_{\mathbf{1}} \otimes_{\mathbb{K}} \mathfrak{g}_{\mathbf{1}}\right)$.

(b) Setting notation $d_{1}:=\operatorname{dim}_{\mathbb{K}}\left(\mathfrak{g}_{1}\right)=|I|$, there exist isomorphisms of (set-valued) functors $\mathbb{A}_{\mathbb{K}}^{0 \mid d_{1}} \cong$ $G_{-}^{\leq}$and $\mathbb{A}_{\mathbb{K}}^{0 \mid d_{1}} \cong \exp \left((-)_{\mathbf{1}} \otimes_{\mathbb{K}} \mathfrak{g}_{1}\right)$, given on $A$-points - for every $A \in(\mathbf{W} \mathbf{s a l g})_{\mathbb{K}}-$ by

$$
\begin{gathered}
\mathbb{A}_{\mathbb{K}}^{0 \mid d_{1}}(A)=A_{\mathbf{1}}^{d_{1}} \longrightarrow G_{-}^{<}(A), \quad\left(\eta_{i}\right)_{i \in I} \mapsto \prod_{i \in I}\left(1+\eta_{i} Y_{i}\right) \\
\mathbb{A}_{\mathbb{K}}^{0 \mid d_{1}}(A)=A_{\mathbf{1}}^{d_{1}} \longrightarrow \exp \left(A_{\mathbf{1}} \otimes_{\mathbb{K}} \mathfrak{g}_{\mathbf{1}}\right), \quad\left(\eta_{i}\right)_{i \in I} \mapsto \exp \left(\sum_{i \in I} \eta_{i} Y_{i}\right)
\end{gathered}
$$


(c) The isomorphisms in (a) and (b) induce further isomorphisms of (set-valued) functors

$$
\begin{aligned}
& N_{G}^{[2]} \times \mathbb{A}_{\mathbb{K}}^{0 \mid d_{1}} \cong G^{-} \quad, \quad N_{G_{0}} \times \mathbb{A}_{\mathbb{K}}^{0 \mid d_{1}} \cong N_{G} \quad, \quad G_{0} \times \mathbb{A}_{\mathbb{K}}^{0 \mid d_{1}} \cong G \\
& \mathbb{A}_{\mathbb{K}}^{0 \mid d_{1}} \times N_{G}^{[2]} \cong G^{-} \quad, \quad \mathbb{A}_{\mathbb{K}}^{0 \mid d_{1}} \times N_{G_{0}} \cong N_{G} \quad, \quad \mathbb{A}_{\mathbb{K}}^{0 \mid d_{1}} \times G_{\mathbf{0}} \cong G
\end{aligned}
$$

Proof. The proof can be done along the same lines followed for Proposition 4.2.11 in [16]. The technical, accessory results that one needs to exploit are the various Lemmas and Propositions developed so far in the present subsection: of course they are formally different, but the overall argument is the same.

\section{From super Harish-Chandra pairs to Lie supergroups}

In this section we provide two different functors $\Psi$ that are quasi-inverse to the functor $\Phi$ of $\S 2.2 .2$. In both cases, for any super Harish-Chandra pair $\mathcal{P}$, we define as associated $\Psi(\mathcal{P}):=G_{\mathcal{P}}$ a suitable functor from Weil superalgebras to groups, and then prove that it has the "right properties". Concretely, we follow the pattern provided by the Global Splitting Theorem for Lie supergroups, which tells us two possible ways how our would-be Lie supergroup $G_{\mathcal{P}}$ should look like: this yields us, eventually, two different recipes.

\subsection{Supergroup functors out of super Harish-Chandra pairs: first recipe}

For later use, we fix the following notation: given $\mathcal{P}=\left(G_{+}, \mathfrak{g}\right) \in(\mathbf{s} \mathbf{H C p})_{\mathbb{K}}, A \in(\mathbf{W} \mathbf{s a l g})_{\mathbb{K}}$ and $c \in A_{\mathbf{0}}$ such that $c^{2}=0$, for every $X \in \mathfrak{g}_{0}$ we set $\left(1_{G_{+}}+c X\right):=\exp (c X) \in G_{+}\left(A_{0}\right)$. When no confusion is possible we will drop the subscript $G_{+}$and simply write $(1+c X)$ instead. Similarly, we shall presently introduce new formal elements of type " $(1+\eta Y)=\exp (\eta Y)$ " with $\eta \in A_{\mathbf{1}}, Y \in \mathfrak{g}_{\mathbf{1}}$.

Definition 3.1.1. Let $\mathcal{P}:=\left(G_{+}, \mathfrak{g}\right) \in(\mathbf{s H C p})_{\mathbb{K}}$ be a super Harish-Chandra pair over $\mathbb{K}$.

(a) We introduce a functor $G_{\mathcal{P}}^{\circ}:(\mathbf{W s a l g})_{\mathbb{K}} \longrightarrow$ (group) as follows. For any Weil superalgebra $A \in(\mathbf{W} \text { salg })_{\mathbb{K}}$, we define $G_{\mathcal{P}}^{\circ}(A)$ as being the group with generators the elements of the set

$$
\Gamma_{A}:=\left\{g_{+},(1+\eta Y) \mid g_{+} \in G_{+}(A),(\eta, Y) \in A_{\mathbf{1}} \times \mathfrak{g}_{1}\right\}=G_{+}(A) \bigcup\{(1+\eta Y)\}_{(\eta, Y) \in A_{1} \times \mathfrak{g}_{1}}
$$

and relations (for $g_{+}^{\prime}, g_{+}^{\prime \prime} \in G_{+}(A), \eta, \eta^{\prime}, \eta^{\prime \prime} \in A_{\mathbf{1}}, Y, Y^{\prime}, Y^{\prime \prime} \in \mathfrak{g}_{\mathbf{1}}, c \in \mathbb{K}$ )

$$
\begin{aligned}
& g_{+}^{\prime} \cdot g_{+}^{\prime \prime}=g_{+\dot{G}_{+}}^{\prime} g_{+}^{\prime \prime}, \quad(1+\eta Y) \cdot g_{+}=g_{+} \cdot\left(1+\eta \operatorname{Ad}\left(g_{+}^{-1}\right)(Y)\right) \\
& \left(1+\eta^{\prime \prime} Y\right) \cdot\left(1+\eta^{\prime} Y\right)=\left(1_{G_{+}}+\eta^{\prime} \eta^{\prime \prime} Y^{\langle 2\rangle}\right)_{G_{+}} \cdot\left(1+\left(\eta^{\prime}+\eta^{\prime \prime}\right) Y\right) \quad, \quad\left(1+\eta 0_{\mathfrak{g}_{1}}\right)=1 \\
& \left(1+\eta^{\prime \prime} Y^{\prime \prime}\right) \cdot\left(1+\eta^{\prime} Y^{\prime}\right)=\left(1_{G_{+}}+\eta^{\prime} \eta^{\prime \prime}\left[Y^{\prime}, Y^{\prime \prime}\right]\right)_{G_{+}} \cdot\left(1+\eta^{\prime} Y^{\prime}\right) \cdot\left(1+\eta^{\prime \prime} Y^{\prime \prime}\right) \quad, \quad\left(1+0_{A} Y\right)=1 \\
& \left(1+\eta Y^{\prime}\right) \cdot\left(1+\eta Y^{\prime \prime}\right)=\left(1+\eta\left(Y^{\prime}+Y^{\prime \prime}\right)\right), \quad(1+(c \eta) Y)=(1+\eta(c Y))
\end{aligned}
$$

where the first line means that for generators chosen in $G_{+}(A)$ their product, denoted ".", inside $G_{\mathcal{P}}^{\circ}(A)$ is the same as in $G_{+}(A)$, where it is denoted “ ${ }_{G_{+}}$"; moreover, $\left(1_{G_{+}}+\eta^{\prime} \eta^{\prime \prime} Y^{\langle 2\rangle}\right)_{G_{+}}$and $\left(1_{G_{+}}+\eta^{\prime} \eta^{\prime \prime}\left[Y^{\prime}, Y^{\prime \prime}\right]\right)_{G_{+}}$ are elements of the form $\left(1_{G_{+}}+c X\right)$ with $X \in \mathfrak{g}_{\mathbf{0}}, c \in A_{\mathbf{0}}$ and $c^{2}=0$ as mentioned above.

Moreover, for any morphism $f: A^{\prime} \longrightarrow A^{\prime \prime}$ in $(\mathbf{W} \text { salg })_{\mathbb{K}}$ we let $G_{\mathcal{P}}^{\circ}(f): G_{\mathcal{P}}^{\circ}\left(A^{\prime}\right) \longrightarrow G_{\mathcal{P}}^{\circ}\left(A^{\prime \prime}\right)$ be the group morphism uniquely defined on generators - for all $g_{+}^{\prime} \in G_{+}\left(A^{\prime}\right), \eta \in A_{1}^{\prime}, Y \in \mathfrak{g}_{1}$ - by

$$
G_{\mathcal{P}}^{\circ}(f)\left(g_{+}^{\prime}\right):=G_{+}(f)\left(g_{+}^{\prime}\right) \quad, \quad G_{\mathcal{P}}^{\circ}(f)\left(1+\eta^{\prime} Y\right):=\left(1+f\left(\eta^{\prime}\right) Y\right)
$$

As the defining relations of each $G_{\mathcal{P}}^{\circ}(A)$ are independent of $A$, such a $G_{\mathcal{P}}^{\circ}(f)$ is well defined indeed.

(b) We define a functor $G_{\mathcal{P}}^{\circ,-}:(\text { Wsalg })_{\mathbb{K}} \longrightarrow($ set $)$ on any object $A \in(\text { Wsalg })_{\mathbb{K}}$ by

$$
G_{\mathcal{P}}^{\circ,-}(A):=\left\{\prod_{s=1}^{n}\left(1+\eta_{s} Y_{s}\right) \mid n \in \mathbb{N},\left(\eta_{s}, Y_{s}\right) \in A_{\mathbf{1}} \times \mathfrak{g}_{\mathbf{1}} \forall s \in\{1, \ldots, n\}\right\} \quad\left(\subseteq G_{\mathcal{P}}^{\circ}(A)\right)
$$

and on morphism in the obvious way - just like for $G_{\mathcal{p}}$. 
(c) Let us fix in $\mathfrak{g}_{\mathbf{1}}$ a $\mathbb{K}$-basis $\left\{Y_{i}\right\}_{i \in I}$ - for some index set $I$ - and a total order in $I$. We define a functor $G_{-}^{<}:(\mathbf{W} \text { salg })_{\mathbb{K}} \longrightarrow($ set $)$ as follows. For $A \in(\mathbf{W s a l g})_{\mathbb{K}}$ we set

$$
G_{-}^{<}(A):=\left\{\vec{\prod}\left(1+\eta_{i} Y_{i}\right) \mid \eta_{i} \in A_{\mathbf{1}} \forall i \in I\right\} \quad\left(\subseteq G_{\mathcal{P}}^{\circ,-}(A) \subseteq G_{\mathcal{P}}^{\circ}(A)\right)
$$

where $\overrightarrow{\prod_{i \in I}}$ denotes an ordered product — with respect to the fixed total order in $I$. This defines the functor $G_{-}^{<}$on objects, and its definition on morphism is the obvious one (like for $G_{\mathcal{P}}^{\circ}$ ).

Remark 3.1.2. By definition, $G_{\mathcal{P}}^{\circ,-}$ and $G_{-}^{<}$are subfunctors of $G_{\mathcal{P}}^{\circ}$. Moreover, every $G_{\mathcal{P}}^{\circ,-}(A)$ is the subgroup of $G_{\mathcal{P}}^{\circ}(A)$ generated by $G_{-}^{\leq}(A)$, or by $\{(1+\eta Y)\}_{(\eta, Y) \in A_{1} \times \mathfrak{g}_{1}}$. Thus, although $G_{-}^{<}$depends on the choice of $\left\{Y_{i}\right\}_{i \in I}$, the supergroup subfunctor that it generates (inside $G_{\mathcal{P}}^{\circ}$ ) instead is independent of that.

Next result shows that $G_{\mathcal{P}}^{\circ}$ can also be described using a much smaller set of generators:

Proposition 3.1.3. Let $\mathcal{P}:=\left(G_{+}, \mathfrak{g}\right) \in(\mathbf{s H C p})_{\mathbb{K}}$, and fix in $\mathfrak{g}_{1}$ a totally ordered $\mathbb{K}$-basis $\left\{Y_{i}\right\}_{i \in I}$. Then for every $A \in(\mathbf{W s a l g})_{\mathbb{K}}$ the group $G_{\mathcal{P}}^{\circ}(A)$ is generated by $\Gamma_{A}^{\bullet}:=G_{+}(A) \bigcup\left\{\left(1+\eta_{i} Y_{i}\right) \mid \eta_{i} \in A_{\mathbf{1}}, \forall i \in I\right\}$.

Proof. Given $A \in(\mathbf{W} \text { salg })_{\mathbb{K}}$, let $G_{\mathcal{P}}^{\bullet}(A)$ be the subgroup of $G_{\mathcal{P}}^{\circ}(A)$ generated by $\Gamma_{A}^{\bullet}$. We will prove that every generator of the group $G_{\mathcal{p}}^{\circ}(A)$ of the form $(1+\eta Y)$ with $(\eta, Y) \in A_{1} \times \mathfrak{g}_{1}$ also belongs to the subgroup $G_{\mathcal{P}}^{\bullet}(A)$ : this then will prove the claim. So let $(\eta, Y) \in A_{\mathbf{1}} \times \mathfrak{g}_{\mathbf{1}}$; then, in terms of the $\mathbb{K}$-basis $\left\{Y_{i}\right\}_{i \in I}$ of $\mathfrak{g}_{\mathbf{1}}$, our $Y$ expands into $Y=\sum_{s=1}^{k} c_{j_{s}} Y_{j_{s}}$. By repeated applications of relations of the form $\left(1+\eta Y^{\prime}\right) \cdot\left(1+\eta Y^{\prime \prime}\right)=\left(1+\eta\left(Y^{\prime}+Y^{\prime \prime}\right)\right)$, we find that the generator $(1+\eta Y)$ in $G_{\mathcal{P}}^{\circ}(A)$ factors as $(1+\eta Y)=\left(1+\eta \sum_{s=1}^{k} c_{j_{s}} Y_{j_{s}}\right)=\prod_{s=1}^{k}\left(1+c_{j_{s}} \eta Y_{j_{s}}\right)$ where the product can be done in any order, as its factors mutually commute. Now the product in right-hand side does belong to $G_{\mathcal{p}}^{\bullet}(A)$, q.e.d.

3.1.4. Another realization of $G_{\mathcal{P}}^{\circ}$. In the following, if $K$ is any group presented by generators and relations, we write $K=\langle\Gamma\rangle /(\mathcal{R})$ if $\Gamma$ is a set of free generators, $\mathcal{R}$ is a set of relations among generators and $(\mathcal{R})$ is the normal subgroup in $K$ generated by $\mathcal{R}$. Note that, given a presentation of $K$ of the form $K=\langle\Gamma\rangle /(\mathcal{R})=\langle\Gamma\rangle /\left(\mathcal{R}_{1} \cup \mathcal{R}_{2}\right)$ with $\mathcal{R}=\mathcal{R}_{1} \cup \mathcal{R}_{2}$, the Double Quotient Theorem gives

$$
K=\langle\Gamma\rangle /(\mathcal{R})=\langle\Gamma\rangle /\left(\mathcal{R}_{1} \cup \mathcal{R}_{2}\right)=\langle\Gamma\rangle /\left(\mathcal{R}_{1}\right) /\left(\mathcal{R}_{1} \cup \mathcal{R}_{2}\right) /\left(\mathcal{R}_{1}\right)=\langle\bar{\Gamma}\rangle /\left(\overline{\mathcal{R}_{2}}\right)
$$

where $\bar{\Gamma}$ and $\overline{\mathcal{R}_{2}}$ respectively denote the images of $\Gamma$ and of $\mathcal{R}_{2}$ in the quotient group $\langle\Gamma\rangle /\left(\mathcal{R}_{1}\right)$.

Let $\mathcal{P}=\left(G_{+}, \mathfrak{g}\right) \in(\mathbf{s H C p})_{\mathbb{K}}$. For any $A \in(\mathbf{W} \text { salg })_{\mathbb{K}}$, we denote by $G_{+}^{[2]}(A)$ the subgroup of $G_{+}(A)$ generated by the set $\left\{(1+c X) \mid c \in A_{1}^{[2]}, X \in\left[\mathfrak{g}_{1}, \mathfrak{g}_{1}\right]\right\}$ - cf. $\S 2.1 .1$ for notation $A_{1}^{[2]}$. Note then that $G_{+}^{[2]}(A)$ is normal in $G_{+}(A)$, as one easily sees by construction (taking into account that, as $\mathcal{P}:=\left(G_{+}, \mathfrak{g}\right)$ is a sHCp, the "adjoint" action of $G_{+}$onto $\mathfrak{g}$ maps $\left[\mathfrak{g}_{1}, \mathfrak{g}_{1}\right]$ into itself). We consider also the three sets

$$
\Gamma_{A}^{+}:=G_{+}(A) \quad, \quad \Gamma_{A}^{[2]}:=G_{+}^{[2]}(A) \quad, \quad \Gamma_{A}^{-}:=\Gamma_{A}^{[2]} \bigcup\{(1+\eta Y)\}_{(\eta, Y) \in A_{1} \times \mathfrak{g}_{1}}
$$

and the sets of relations - for all $g_{+}, g_{+}^{\prime}, g_{+}^{\prime \prime} \in \Gamma_{A}^{+}, g_{[2]}, g_{[2]}^{\prime}, g_{[2]}^{\prime \prime} \in \Gamma_{A}^{[2]}, \eta, \eta^{\prime}, \eta^{\prime \prime} \in A_{\mathbf{1}}, X \in\left[\mathfrak{g}_{1}, \mathfrak{g}_{1}\right]$, $Y, Y^{\prime}, Y^{\prime \prime} \in \mathfrak{g}_{\mathbf{1}}$, with $\dot{G}_{+}$and $\dot{G}_{+}^{[2]}$ being the product in $G_{+}(A)$ and in $G_{+}^{[2]}(A)$ - given by

$$
\begin{array}{ccc}
\mathcal{R}_{A}^{+}: \quad g_{+}^{\prime} \cdot g_{+}^{\prime \prime}=g_{+\dot{G}_{+}}^{\prime} g_{+}^{\prime \prime}, & \mathcal{R}_{A}^{[2]}: \quad\left(g_{[2]}\right)_{\Gamma_{A}^{[2]}}=\left(g_{[2]}\right)_{\Gamma_{A}^{+}} \\
\mathcal{R}_{A}^{\ltimes}: & g_{[2]} \cdot g_{+}=g_{+} \cdot\left(g_{+}^{-1} \dot{G}_{+} g_{[2] \dot{G}_{+}} g_{+}\right), & (1+\eta Y) \cdot g_{+}=g_{+} \cdot\left(1+\eta A d\left(g_{+}^{-1}\right)(Y)\right)
\end{array}
$$




$$
\mathcal{R}_{A}^{-}:\left\{\begin{array}{c}
g_{[2]}^{\prime} \cdot g_{[2]}^{\prime \prime}=g_{[2]}^{\prime} \dot{G}_{+}^{[2]} g_{[2]}^{\prime \prime} \\
\left(1+\eta^{\prime \prime} Y\right) \cdot\left(1+\eta^{\prime} Y\right)=\left(1+\eta^{\prime} \eta^{\prime \prime} Y^{\langle 2\rangle}\right) \cdot\left(1+\left(\eta^{\prime}+\eta^{\prime \prime}\right) Y\right) \\
(1+\eta Y) \cdot g_{[2]}=g_{[2]} \cdot\left(1+\eta A d\left(g_{[2]}^{-1}\right)(Y)\right) \\
\left(1+\eta^{\prime \prime} Y^{\prime \prime}\right) \cdot\left(1+\eta^{\prime} Y^{\prime}\right)=\left(1+\eta^{\prime} \eta^{\prime \prime}\left[Y^{\prime}, Y^{\prime \prime}\right]\right) \cdot\left(1+\eta^{\prime} Y^{\prime}\right) \cdot\left(1+\eta^{\prime \prime} Y^{\prime \prime}\right) \\
\left(1+\eta Y^{\prime}\right) \cdot\left(1+\eta Y^{\prime \prime}\right)=\left(1+\eta\left(Y^{\prime}+Y^{\prime \prime}\right)\right) \\
\left(1+\eta 0_{\mathfrak{g}_{1}}\right)=1, \quad\left(1+0_{A} Y\right)=1 \\
\mathcal{R}_{A}:=\mathcal{R}_{A}^{+} \cup \mathcal{R}_{A}^{-} \cup \mathcal{R}_{A}^{\ltimes} \cup \mathcal{R}_{A}^{[2]}
\end{array}\right.
$$

(in particular, note that the relations of type $\mathcal{R}_{A}^{[2]}$ just identify each element in $\Gamma_{A}^{[2]}$ with its copy inside $\left.\Gamma_{A}^{+}\right)$. Then we define a new group, by generators and relations, namely $G_{\mathcal{P}}^{\circ,-}(A):=\left\langle\Gamma_{A}^{-}\right\rangle /\left(\mathcal{R}_{A}^{-}\right)$.

From the very definition of $G_{\mathcal{P}}^{\circ}(A)$ - cf. Definition 3.1.1 — it follows that

$$
G_{\mathcal{P}}^{\circ}(A) \cong\left\langle\Gamma_{A}^{+} \bigcup \Gamma_{A}^{-}\right\rangle /\left(\mathcal{R}_{A}\right)=\left\langle\Gamma_{A}^{+} \bigcup \Gamma_{A}^{-}\right\rangle /\left(\mathcal{R}_{A}^{+} \bigcup \mathcal{R}_{A}^{-} \bigcup \mathcal{R}_{A}^{\ltimes} \bigcup \mathcal{R}_{A}^{[2]}\right)
$$

indeed, here above we are just taking larger sets of generators and of relations (w.r.t. Definition 3.1.1), but with enough redundancies as to find a different presentation of the same group.

From this we find a neat description of $G_{\mathcal{P}}^{\circ}(A)$ by achieving the presentation (3.2) in a series of intermediate steps, namely adding only one bunch of relations at a time. As a first step, we have

$$
\left\langle\Gamma_{A}^{+} \bigcup \Gamma_{A}^{-}\right\rangle /\left(\mathcal{R}_{A}^{+} \bigcup \mathcal{R}_{A}^{-}\right)=\left\langle\Gamma_{A}^{+}\right\rangle /\left(\mathcal{R}_{A}^{+}\right) *\left\langle\Gamma_{A}^{-}\right\rangle /\left(\mathcal{R}_{A}^{-}\right) \cong G_{+}(A) * G_{\mathcal{P}}^{\circ,-}(A)
$$

where $G_{+}(A) \cong\left\langle\Gamma_{A}^{+}\right\rangle /\left(\mathcal{R}_{A}^{+}\right)$by construction and $*$ denotes the free product (of two groups).

For the next two steps we can follow two different lines of action. On the one hand, (3.1) and (3.3) give $\left\langle\Gamma_{A}^{+} \cup \Gamma_{A}^{-}\right\rangle /\left(\mathcal{R}_{A}^{+} \cup \mathcal{R}_{A}^{-} \cup \mathcal{R}_{A}^{\ltimes}\right) \cong\left(G_{+}(A) * G_{\mathcal{P}}^{\circ,-}(A)\right) /\left(\overline{\mathcal{R}_{A}^{\ltimes}}\right) \cong G_{+}(A) \ltimes G_{\mathcal{P}}^{\circ,-}(A)$, where $G_{+}(A) \ltimes G_{\mathcal{P}}^{\circ,-}(A)$ is the semidirect product of $G_{+}(A)$ with $G_{\mathcal{P}}^{\circ,-}(A)$ w. r. to the "adjoint" action of the former on the latter. Then $G_{\mathcal{P}}^{\circ}(A) \cong\left\langle\Gamma_{A}^{+} \cup \Gamma_{A}^{-}\right\rangle /\left(\mathcal{R}_{A}\right) \cong\left\langle\Gamma_{A}^{+} \cup \Gamma_{A}^{-}\right\rangle /\left(\mathcal{R}_{A}^{+} \cup \mathcal{R}_{A}^{-} \cup \mathcal{R}_{A}^{\ltimes} \cup \mathcal{R}_{A}^{[2]}\right) \cong$ $\cong\left(G_{+}(A) \ltimes G_{\mathcal{P}}^{\circ,-}(A)\right) /\left(\overline{\mathcal{R}_{A}^{[2]}}\right) \cong\left(G_{+}(A) \ltimes G_{\mathcal{P}}^{\circ,-}(A)\right) / N_{[2]}(A)$ where $N_{[2]}(A)$ is the normal subgroup of $G_{+}(A) \ltimes G_{\mathcal{P}}^{\circ,-}(A)$ generated by $\left\{\left(g_{[2]}, g_{[2]}^{-1}\right)\right\}_{g_{[2]} \in \Gamma_{A}^{[2]}}$. Thus $G_{\mathcal{P}}^{\circ}(A)=\left(G_{+}(A) \ltimes G_{-}(A)\right) / N_{[2]}(A)$.

Similarly, we find also that $\left\langle\Gamma_{A}^{+} \cup \Gamma_{A}^{-}\right\rangle /\left(\mathcal{R}_{A}^{+} \cup \mathcal{R}_{A}^{-} \cup \mathcal{R}_{A}^{[2]}\right) \cong\left(G_{+}(A) * G_{\mathcal{P}}^{\circ,-}(A)\right) /\left(\overline{\mathcal{R}_{A}^{[2]}}\right) \cong$ $\cong G_{+}(A) *_{G_{+}^{[2]}(A)} G_{\mathcal{P}}^{\circ,-}(A)$ where $G_{+}(A) \underset{G_{+}^{[2]}(A)}{*} G_{\mathcal{P}}^{\circ,-}(A)$ is the amalgamated product of $G_{+}(A)$ and $G_{\mathcal{P}}^{\circ,-}(A)$ over $G_{+}^{[2]}(A)$ w.r.t. the natural monomorphisms $G_{+}^{[2]}(A) \longleftrightarrow G_{+}(A)$ and $G_{+}^{[2]}(A) \longleftrightarrow G_{\mathcal{P}}^{\circ,-}(A)$. Then

$$
\begin{aligned}
G_{\mathcal{P}}^{\circ}(A) \cong\left\langle\Gamma_{A}^{+} \cup \Gamma_{A}^{-}\right\rangle /\left(\mathcal{R}_{A}\right) \cong\left\langle\Gamma_{A}^{+} \cup \Gamma_{A}^{-}\right\rangle /\left(\mathcal{R}_{A}^{+} \cup \mathcal{R}_{A}^{-} \cup \mathcal{R}_{A}^{[2]} \cup \mathcal{R}_{A}^{\ltimes}\right) \cong \\
\cong\left(G_{+}(A) \underset{G_{+}^{[2]}(A)}{*} G_{\mathcal{P}}^{\circ,-}(A)\right) /\left(\overline{\mathcal{R}_{A}^{\ltimes}}\right) \cong\left(G_{+}(A) \underset{G_{+}^{[2]}(A)}{*} G_{\mathcal{P}}^{\circ,-}(A)\right) / N_{\ltimes}(A)
\end{aligned}
$$

where $N_{\ltimes}(A)$ is the normal subgroup of $G_{+}(A) \underset{G_{+}^{[2]}(A)}{*} G_{\mathcal{P}}^{\circ,-}(A)$ generated by

$$
\left\{g_{+}(1+\eta Y) g_{+}^{-1}\left(1+\eta A d\left(g_{+}\right)(Y)\right)^{-1}\right\}_{(\eta, Y) \in A_{\mathbf{1}} \times \mathfrak{g}_{1}}^{g_{+} \in G_{+}(A)} \bigcup\left\{g_{+} g_{[2]} g_{+}\left(g_{+} \dot{G}_{+} g_{[2] \dot{G}_{+}} g_{+}\right)^{-1}\right\}_{g_{+} \in G_{+}(A)}^{g_{[2]} \in \Gamma_{A}^{[2]}}
$$


Thus $G_{\mathcal{P}}^{\circ}(A)=\left(G_{+}(A) \underset{G_{+}^{[2]}(A)}{*} G_{\mathcal{P}}^{\circ,-}(A)\right) / N_{\ltimes}(A)$ for all $A \in(\mathbf{W} \text { salg })_{\mathbb{K}}$; in functorial terms, this reads

$$
G_{\mathcal{P}}^{\circ}=\left(G_{+} \ltimes G_{\mathcal{P}}^{\circ,-}\right) / N_{[2]} \quad \text { and } \quad G_{\mathcal{P}}^{\circ}=\left(G_{+}^{*} \underset{G_{+}^{[2]}}{*} G_{\mathcal{P}}^{\circ,-}\right) / N_{\ltimes} \quad, \quad \text { or } \quad G_{\mathcal{P}}^{\circ}=G_{+} \underset{G_{+}^{[2]}}{\ltimes} G_{\mathcal{P}}^{\circ,-}
$$

where the last, (hopefully) more suggestive notation $G_{\mathcal{P}}^{\circ}=G_{+} \underset{G^{[2]}}{\ltimes} G_{\mathcal{P}}^{\circ,-}$ tells us that $G_{\mathcal{P}}^{\circ}$ is the "amalgamate semidirect product" of $G_{+}$and $G_{\mathcal{P}}^{\circ,-}$ over their common subgroup $G_{+}^{[2]}$.

\subsection{The supergroup functor $G_{\mathcal{P}}^{\circ}$ as a Lie supergroup}

We aim now to proving that the functor $G_{\mathcal{p}}^{\circ}$ is actually a Lie supergroup. We keep notations as before, in particular for every $A \in(\mathbf{W} \text { salg })_{\mathbb{K}}$ we denote by $G_{+}^{[2]}(A)$ the subgroup of $G_{+}(A)$ generated by $\{(1+c X)\}_{X \in\left[\mathfrak{g}_{1}, \mathfrak{g}_{1}\right]}^{c \in A^{[2]}}$ - cf. $\S 2.1 .1$ for notation $A_{1}^{[2]}$. We begin with a "factorization result" for $G_{\mathcal{P}}^{\circ}$ :

Proposition 3.2.1. Let $\mathcal{P}:=\left(G_{+}, \mathfrak{g}\right) \in(\mathbf{s H C p})_{\mathbb{K}}$ be a super Harish-Chandra pair over $\mathbb{K}$, let $\left\{Y_{i}\right\}_{i \in I}$ be a totally ordered $\mathbb{K}$-basis of $\mathfrak{g}_{1}$ (for our fixed order in $I$ ) and $A \in(\mathbf{W} \text { salg })_{\mathbb{K}}$. Then:

(a) letting $\left\langle G_{-}^{<}(A)\right\rangle$ be the subgroup of $G_{\mathcal{P}}^{\circ}(A)$ generated by $G_{-}^{<}(A)$, we have $\left\langle G_{-}^{<}(A)\right\rangle=G_{\mathcal{P}}^{\circ,-}(A)$ and there exist set-theoretic factorizations (with respect to the group product ".")

$$
G_{\mathcal{P}}^{\circ,-}(A)=G_{+}^{[2]}(A) \cdot G_{-}^{<}(A) \quad, \quad G_{\mathcal{P}}^{\circ,-}(A)=G_{-}^{<}(A) \cdot G_{+}^{[2]}(A)
$$

(b) there exist set-theoretic factorizations (with respect to the group product ".")

$$
G_{\mathcal{P}}^{\circ}(A)=G_{+}(A) \cdot G_{-}^{\leq}(A) \quad, \quad G_{\mathcal{P}}^{\circ}(A)=G_{-}^{<}(A) \cdot G_{+}(A)
$$

Proof. Claim (a) is the exact analogue of (2.5), and claim (b) the analogue of (2.7), in Proposition 2.4.8(b). In both cases the proof (up to trivialities) is identical, so we can skip it.

3.2.2. The representation $G_{\mathcal{P}}^{\circ} \longrightarrow \mathrm{GL}(V)$. When discussing the structure of a Lie supergroup $G$, the factorization $G=G_{\mathbf{0}} \cdot G_{-}^{<}$was just a intermediate step; Proposition 3.2.1 above gives us the parallel counterpart for $G_{\mathcal{p}}^{\circ}$. This factorization result for $G$ is improved by the "Global Splitting Theorem" i.e. Theorem 2.4.10 - that, roughly speaking, states that for any $g \in G(A)$ the factorization pertaining to $G_{\mathbf{0}}(A) \cdot G_{-}^{\leq}(A)$ has uniquely determined factors, and similarly any element in $G_{-}^{\leq}(A)$ has a unique factorization into an ordered product of factors of the form $\left(1+\eta_{i} Y_{i}\right)$. Both results are proved by showing that two factorizations of the same object necessarily have identical factors; in other words, distinct choices of factors always give rise to different elements in $G(A)$ or in $G_{-}^{\leq}(A)$. This last fact was proved using the concrete realization of $G(A)$ as a special set of maps, namely $G(A):=\bigsqcup_{x \in|G|} \operatorname{Hom}_{(\mathbf{s a l g})_{\mathbb{K}}}\left(\mathcal{O}_{|G|, x}, A\right)$; indeed, this algebra is rich enough to "separate" different elements of $G(A)$ itself just looking at their values as $A$ valued maps. When dealing with $G_{\mathcal{P}}^{\circ}(A)$ instead, that is defined abstractly, such a built-in realization is not available: our strategy then is to replace it with a suitable "partial linearization", namely a representation of $G_{\mathcal{P}}^{\circ}(A)$ that, although not being faithful, is still "rich enough" to (almost) separate elements.

Let $\mathcal{P}=\left(G_{+}, \mathfrak{g}\right) \in(\mathbf{s H C p})_{\mathbb{K}}$ be given; as before, we fix a $\mathbb{K}$-basis $\left\{Y_{i}\right\}_{i \in I}$ of $\mathfrak{g}$, where $I$ is an index set in which we fix some total order, hence the basis itself is totally ordered as well.

Recall that the universal enveloping algebra $U(\mathfrak{g})$ is given by $U(\mathfrak{g}):=T(\mathfrak{g}) / J$ where $T(\mathfrak{g})$ is the tensor algebra of $\mathfrak{g}$ and $J$ is the two-sided ideal in $T(\mathfrak{g})$ generated by the set (using notation $z^{\langle 2\rangle}:=2^{-1}[z, z]$ )

$$
\left\{x y-(-1)^{|x||y|} y x-[x, y], z^{2}-z^{\langle 2\rangle} \mid x, y \in \mathfrak{g}_{\mathbf{0}} \bigcup \mathfrak{g}_{\mathbf{1}}, z \in \mathfrak{g}_{\mathbf{1}}\right\}
$$

It is known then - see for instance [24], §7.2 - that one has splitting(s) of $\mathbb{K}$-supercoalgebras

$$
U(\mathfrak{g}) \cong U\left(\mathfrak{g}_{0}\right) \otimes_{\mathbb{K}} \wedge \mathfrak{g}_{1} \cong \wedge \mathfrak{g}_{1} \otimes_{\mathbb{K}} U\left(\mathfrak{g}_{0}\right)
$$

and $\wedge \mathfrak{g}_{1}$ has $\mathbb{K}$-basis $\left\{Y_{i_{1}} \wedge Y_{i_{2}} \wedge \cdots \wedge Y_{i_{s}}|s \leqslant| I \mid, i_{1}<i_{2}<\cdots<i_{s}\right\}$. 
Now let $\mathbf{1}$ be the (one-dimensional) trivial representation of $\mathfrak{g}_{\mathbf{0}}$. By induction from $\mathfrak{g}_{\mathbf{0}}$ to $\mathfrak{g}$ we can consider the induced representation $V:=\operatorname{Ind}_{\mathfrak{g}_{0}}^{\mathfrak{g}}(\mathbf{1})$, that is a $\mathfrak{g}$-module. Looking at $\mathbf{1}$ and $V$ respectively as a module over $U\left(\mathfrak{g}_{\mathbf{0}}\right)$ and over $U(\mathfrak{g})$, taking (3.4) into account we get

$$
V:=\operatorname{Ind}_{\mathfrak{g}_{0}}^{\mathfrak{g}}(\mathbf{1})=U(\mathfrak{g}) \underset{U\left(\mathfrak{g}_{0}\right)}{\otimes} \mathbf{1} \cong \bigwedge \mathfrak{g}_{\mathbf{1}} \underset{\mathbb{K}}{\otimes} \mathbf{1} \cong \bigwedge \mathfrak{g}_{1}
$$

The last one above is a natural isomorphism of $\mathbb{K}$-superspaces, uniquely determined once a specific element $\underline{b} \in \mathbf{1}$ is fixed to form a $\mathbb{K}$-basis of $\mathbf{1}$ itself: the isomorphism is $\omega \otimes \underline{b} \mapsto \omega$ for all $\omega \in \bigwedge \mathfrak{g}_{\mathbf{1}}$.

This and its outcome give rise to similar functorial counterparts, for the Lie algebra valued $\mathbb{K}$-superfunctors $\mathcal{L}_{\mathfrak{g}_{0}}$ and $\mathcal{L}_{\mathfrak{g}}$, as well as for the $\mathbb{K}$-superfunctors associated with $U\left(\mathfrak{g}_{\mathbf{0}}\right)$ and $U(\mathfrak{g})$, in the standard way, namely $A \mapsto A_{\mathbf{0}} \otimes_{\mathbb{K}} U\left(\mathfrak{g}_{\mathbf{0}}\right)$ and $A \mapsto\left(A \otimes_{\mathbb{K}} U(\mathfrak{g})\right)_{\mathbf{0}}=A_{\mathbf{0}} \otimes_{\mathbb{K}} U(\mathfrak{g})_{\mathbf{0}} \oplus A_{\mathbf{1}} \otimes_{\mathbb{K}} U(\mathfrak{g})_{\mathbf{1}}$ for all $A \in(\mathbf{W s a l g})_{\mathbb{K}}$.

On the other hand $\mathfrak{g}_{0}=\operatorname{Lie}\left(G_{+}\right)$, and $\mathbf{1}$ is also the trivial representation for $G_{+}$, as a classical Lie group. Then the representation of $\mathfrak{g}$ on the space $V$ also induces a representation of the super Harish-Chandra pair $\mathcal{P}=\left(G_{+}, \mathfrak{g}\right)$ on the same $V$, in other words $V$ bears also a structure of $\left(G_{+}, \mathfrak{g}\right)$-module - in the obvious sense: we have a morphism $\left(\boldsymbol{r}_{+}, \rho\right):\left(G_{+} \mathfrak{g}\right) \longrightarrow(\mathrm{GL}(V), \mathfrak{g l}(V))$ of super Harish-Chandra pairs. We will write again $\rho$ for the representation map $\rho: U(\mathfrak{g}) \longrightarrow \operatorname{End}_{\mathbb{K}}(V)$ giving the $U(\mathfrak{g})$-module structure on $V$.

Our key step now is that the above $\left(G_{+}, \mathfrak{g}\right)$-module structure on $V$ "integrates" to a $G_{\mathcal{P}}^{\circ}$-module structure.

Proposition 3.2.3. Retain notation as above for the $\left(G_{+}, \mathfrak{g}\right)$-module $V$. There exists a unique structure of (left) $G_{\mathcal{P}}^{\circ}$-module onto $V$ which satisfies the following conditions: for every $A \in(\mathbf{W s a l g})_{\mathbb{K}}$, the representation map $\boldsymbol{r}_{\mathcal{P}, A}^{\circ}: G_{\mathcal{P}}^{\circ}(A) \longrightarrow \mathrm{GL}(V)(A)$ is given on generators of $G_{\mathcal{P}}^{\circ}(A)$ - namely, all $g_{+} \in G_{+}(A)$ and $\left(1+\eta_{i} Y_{i}\right)$ for $i \in I, \eta_{i} \in A_{\mathbf{1}}-b y \boldsymbol{r}_{\mathcal{P}, A}^{\circ}\left(g_{+}\right):=\boldsymbol{r}_{+}\left(g_{+}\right), \quad \boldsymbol{r}_{\mathcal{P}, A}^{\circ}\left(1+\eta_{i} Y_{i}\right):=\rho\left(1+\eta_{i} Y_{i}\right)=i d_{V}+\eta_{i} \rho\left(Y_{i}\right)$, or in other words $g_{+} \cdot v:=\boldsymbol{r}_{+}\left(g_{+}\right)(v)$ and $\left(1+\eta_{i} Y_{i}\right) \cdot v:=\rho\left(1+\eta_{i} Y_{i}\right)(v)=v+\eta_{i} \rho\left(Y_{i}\right)(v)$ for all $v \in V(A)$. Overall, this yields a morphism a $\mathbb{K}$-supergroup functors $\boldsymbol{r}_{\mathcal{P}}^{\circ}: G_{\mathcal{P}}^{\circ} \longrightarrow \mathrm{GL}(V)$.

Proof. This follows from the whole construction, and from the very definition of $G_{\mathcal{P}}^{\circ}$. Indeed, by definition of representation for the super Harish-Chandra pair $\mathcal{P}$ we see that the operators $\boldsymbol{r}_{\mathcal{P}, A}^{\circ}\left(g_{+}\right)$and $\boldsymbol{r}_{\mathcal{P}, A}^{\circ}\left(1+\eta_{i} Y_{i}\right)$ on $V$ - associated with the generators of $G_{\mathcal{P}}^{\circ}(A)$ - do satisfy all relations which, by Definition 3.1.1(a), are satisfied by the generators themselves. Thus they uniquely provide a well-defined group morphism $\boldsymbol{r}_{\mathcal{P}, A}^{\circ}: G_{\mathcal{P}}^{\circ}(A) \longrightarrow \mathrm{GL}(V)(A)$ as required. The construction is clearly functorial in $A$, whence the claim.

The advantage of introducing the representation $\boldsymbol{r}_{\mathcal{P}}$ of $G_{\mathcal{P}}^{\circ}$ on $V$ is that it allows us to "separate" the "odd points of $G_{\mathcal{P}}^{\circ}(A)$ from each other and from the even ones".

We are now ready to state and prove the "global splitting theorem" for $G_{\mathcal{P}}^{\circ}$ (cf. Theorem 2.4.10):

\section{Proposition 3.2.4.}

(a) The restriction of group multiplication in $G_{\mathcal{P}}^{\circ}$ provides isomorphisms of (set-valued) functors

$$
G_{+} \times G_{-}^{<} \cong G_{\mathcal{P}}^{\circ}, \quad G_{-}^{<} \times G_{+} \cong G_{\mathcal{P}}^{\circ} \quad, \quad G_{+}^{[2]} \times G_{-}^{<} \cong G_{\mathcal{P}}^{\circ,-}, \quad G_{-}^{<} \times G_{+}^{[2]} \cong G_{\mathcal{P}}^{\circ,-}
$$

(b) There exists an isomorphism of (set-valued) functors $\mathbb{A}_{\mathbb{K}}^{0 \mid d_{1}} \cong G_{-}^{<}$, with $d_{1}:=|I|=\operatorname{dim}_{\mathbb{K}}\left(\mathfrak{g}_{1}\right)$, given on $A$-points - for every $A \in(\mathbf{W s a l g})_{\mathbb{K}}-b y$

$$
\mathbb{A}_{\mathbb{K}}^{0 \mid d_{1}}(A)=A_{1}^{d_{1}} \longrightarrow G_{-}^{<}(A), \quad\left(\eta_{i}\right)_{i \in I} \mapsto \prod_{i \in I}\left(1+\eta_{i} Y_{i}\right)
$$

(c) There exist isomorphisms of (set-valued) functors

$$
G_{+} \times \mathbb{A}_{\mathbb{K}}^{0 \mid d_{1}} \cong G_{\mathcal{P}}^{\circ}, \quad G_{+}^{[2]} \times \mathbb{A}_{\mathbb{K}}^{0 \mid d_{1}} \cong G_{\mathcal{P}}^{\circ,-}, \quad \text { and } \quad \mathbb{A}_{\mathbb{K}}^{0 \mid d_{1}} \times G_{+} \cong G_{\mathcal{P}}^{\circ}, \quad \mathbb{A}_{\mathbb{K}}^{0 \mid d_{1}} \times G_{+}^{[2]} \cong G_{\mathcal{P}}^{\circ,-}
$$

given on $A$-points - for every $A \in(\mathbf{W} \text { salg })_{\mathbb{K}}$ - respectively by

$$
\left(g_{+},\left(\eta_{i}\right)_{i \in I}\right) \mapsto g_{+} \cdot \overrightarrow{\prod_{i \in I}}\left(1+\eta_{i} Y_{i}\right) \quad \text { and } \quad\left(\left(\eta_{i}\right)_{i \in I}, g_{+}\right) \mapsto \prod_{i \in I}\left(1+\eta_{i} Y_{i}\right) \cdot g_{+}
$$


Proof. The proof is quite close to (half of) that of Theorem 2.4.10, the difference being the use of the representation $V$ of $§ 3.2 .2$. For completeness we present it shortly, sketching ( $a$ ) and (b), whence (c) follows.

(a) It is enough to prove the first identity for $G_{\mathcal{P}}^{\circ}$ (all other are similar). Thus our claim is: for any $A \in(\mathbf{W} \text { salg })_{\mathbb{K}}$, if $\hat{g}_{+} \hat{g}_{-}=\check{g}_{+} \check{g}_{-}$for $\hat{g}_{+}, \check{g}_{+} \in G_{+}(A)$ and $\hat{g}_{-}, \check{g}_{-} \in G_{-}^{<}(A)$, then $\hat{g}_{+}=\check{g}_{+}$and $\hat{g}_{-}=\check{g}_{-}$.

From $\hat{g}_{+} \hat{g}_{-}=\check{g}_{+} \check{g}_{-}$we get $g:=\hat{g}_{-} \check{g}_{-}^{-1}=\hat{g}_{+}^{-1} \check{g}_{+} \in G_{+}(A)$, as $G_{+}(A)$ is a subgroup. Writing $\hat{g}_{-}=\vec{\prod}_{i \in I}\left(1+\hat{\eta}_{i} Y_{i}\right)$ and $\check{g}_{-}=\vec{\prod}_{i \in I}\left(1+\check{\eta}_{i} Y_{i}\right)$ we get $g:=\hat{g}_{-} \check{g}_{-}^{-1}=\prod_{i \in I}\left(1+\hat{\eta}_{i} Y_{i}\right) \overleftarrow{\prod_{i \in I}}\left(1-\check{\eta}_{i} Y_{i}\right) \in G_{+}(A)$

Let $\mathfrak{a}:=\left(\left\{\hat{\eta}_{i}, \check{\eta}_{i}\right\}_{i \in I}\right)$ be the ideal of $A$ generated by the $\hat{\eta}_{i}$ 's and the $\check{\eta}_{i}$ 's, set $A \stackrel{\pi_{n}}{\longrightarrow} A / \mathfrak{a}^{n}$ for the quotient map and $[a]_{n}:=\pi_{n}(a)$ for $a \in A$, then $G_{\mathcal{P}}^{\circ}(A) \stackrel{G\left(\pi_{n}\right)}{\longrightarrow} G_{\mathcal{P}}^{\circ}\left(A / \mathfrak{a}^{n}\right)$ for the associated group morphism and $[y]_{n}:=G_{\mathcal{P}}^{\circ}\left(\pi_{n}\right)(y)$ for every $y \in G_{\mathcal{P}}^{\circ}(A)$. Now, the defining relations for $G_{-}^{<}\left(A / \mathfrak{a}^{2}\right)$ yield $[g]_{2}=\overrightarrow{\prod_{i \in I}}\left(1+\left[\hat{\eta}_{i}\right]_{2} Y_{i}\right) \cdot \prod_{i \in I}^{\overleftarrow{ }}\left(1-\left[\check{\eta}_{i}\right]_{2} Y_{i}\right)=\prod_{i \in I}^{\rightarrow}\left(1+\left[\alpha_{i}\right]_{2} Y_{i}\right) \in G_{-}^{<}\left(A / \mathfrak{a}^{2}\right)$ with $\alpha_{i}:=\hat{\eta}_{i}-\check{\eta}_{i} \quad(\forall i \in I)$.

Next step then is to let $[g]_{2}$ act onto $\underline{b} \in V\left(A / \mathfrak{a}^{2}\right)$; we write $\bar{Y}$ for the image of $Y . b$ through the isomorphism $V=\bigwedge \mathfrak{g}_{\mathbf{1}} \cdot \underline{b} \cong \bigwedge \mathfrak{g}_{\mathbf{1}}$. Then $[g]_{2} \cdot \underline{b}=\prod_{i \in I}^{\rightarrow}\left(1+\left[\alpha_{i}\right]_{2} Y_{i}\right) \cdot \underline{b}=\underline{b}+\sum_{i \in I}\left[\alpha_{i}\right]_{2} \bar{Y}_{i} \in V\left(A / \mathfrak{a}^{2}\right)$ since $\left[\alpha_{h}\right]_{2}\left[\alpha_{k}\right]_{2}=[0]_{2} \in A / \mathfrak{a}^{2}$; on the other hand, $[g]_{2} \cdot \underline{b}=\underline{b}$ as $[g]_{2} \in G_{+}\left(A / \mathfrak{a}^{2}\right)$ and $G_{+}$acts trivially on $V$. Therefore, noting that $\{\underline{b}\} \cup\left\{\bar{Y}_{i}\right\}_{i \in I}$ is part of the chosen basis of $V$, we get $\left[\alpha_{i}\right]_{2}=[0]_{2} \in A / \mathfrak{a}^{2}$, i.e. $\alpha_{i} \in \mathfrak{a}^{2}$, for all $i \in I$. By the same argument we find $[g]_{3}=\prod_{i \in I}\left(1+\left[\alpha_{i}\right]_{3} Y_{i}\right) \in G_{\mathcal{P}}^{\circ}\left(A / \mathfrak{a}^{3}\right)$ hence $[g]_{3} \cdot \underline{b}=\underline{b}+\sum_{i \in I}\left[\alpha_{i}\right]_{3} \bar{Y}_{i} \in V\left(A / \mathfrak{a}^{3}\right)$, which in turn implies $\alpha_{i} \in \mathfrak{a}^{3}$, for all $i \in I$. Iterating this process we find $\alpha_{i} \in \mathfrak{a}^{n}$ for all $n \in \mathbb{N}, i \in I$; as $\mathfrak{a}^{n}=\{0\}$ for $n \gg 0$ (since $\mathfrak{a}$ is generated by finitely many odd elements) we end up with $\alpha_{i}=0$, i.e. $\hat{\eta}_{i}=\check{\eta}_{i}$, for all $i \in I$. This means $\hat{g}_{-}=\check{g}_{-}$, whence $\hat{g}_{+}=\check{g}_{+}$too.

(b) By construction there exists a morphism $\Theta^{<}: \mathbb{A}_{\mathbb{K}}^{0 \mid d_{1}} \longrightarrow G_{-}^{<}$of set-valued functors given on $A^{-}$ points - for $A \in(\mathbf{W s a l g})_{\mathbb{K}}$ — by the map $\Theta_{A}^{<}: \mathbb{A}_{\mathbb{K}}^{0 \mid d_{1}}(A):=A_{1}^{\times d_{1}} \longrightarrow G_{-}^{<}(A),\left(\eta_{i}\right)_{i \in I} \mapsto \overrightarrow{\prod_{i \in I}}\left(1+\eta_{i} Y_{i}\right)$, that is actually surjective, and we need to prove that is also injective. Now, let $\left(\hat{\eta}_{i}\right)_{i \in I},\left(\check{\eta}_{i}\right)_{i \in I} \in A_{\mathbf{1}}^{\times d_{1}}$ be such that $\Theta_{A}^{<}\left(\left(\hat{\eta}_{i}\right)_{i \in I}\right)=\Theta_{A}^{<}\left(\left(\check{\eta}_{i}\right)_{i \in I}\right)$, i.e. $\prod_{i \in I}\left(1+\hat{\eta}_{i} Y_{i}\right)=\overrightarrow{\prod_{i \in I}}\left(1+\check{\eta}_{i} Y_{i}\right)$. Then we repeat the argument in $(a)$, now with $\hat{g}_{+}:=1=: \check{g}_{+}$, which gives $\hat{\eta}_{i}=\check{\eta}_{i}$ for all $i \in I$, i.e. $\left(\hat{\eta}_{i}\right)_{i \in I}=\left(\check{\eta}_{i}\right)_{i \in I}$, q.e.d.

3.2.5. The Lie supergroup structure of $G_{\mathcal{P}}^{\circ}$. Given $\mathcal{P} \in(\mathbf{s H C p})_{\mathbb{K}}$ and $A \in(\mathbf{W s a l g})_{\mathbb{K}}$, Proposition $3.2 .4(c)$ yields a bijection $\phi_{A}^{\circ}: G_{+}(A) \times \mathbb{A}_{\mathbb{K}}^{0 \mid d_{1}}(A) \cong \cong G_{\mathcal{P}}^{\circ}(A)$ whose restriction to $G_{+}(A)$, identified with $G_{+}(A) \times\left\{(0)_{i \in I}\right\}$, is the identity — onto the copy of $G_{+}(A)$ naturally sitting inside $G_{\mathcal{P}}^{\circ}(A)$.

Now, $G_{+}(A)$ is by definition an $A_{0^{-}}$manifold (cf. [3]), of the same type (real smooth, etc.) as the sHCp $\mathcal{P}:=\left(G_{+}, \mathfrak{g}\right)$ it pertains to; on the other hand, $\mathbb{A}_{\mathbb{K}}^{0 \mid d_{1}}(A)$ carries natural, canonical structures of $A_{0^{-}}$manifold of any possible type (real smooth or real analytic if $\mathbb{K}=\mathbb{R}$, complex holomorphic if $\mathbb{K}=\mathbb{C}$ ), in particular then also of the type of $G_{+}(A)$. Then we know that there is also a canonical "product structure" of $A_{0^{-}}$ manifold - of the same type of $G_{+}(A)$, i.e. of $\mathcal{P}$ — onto the Cartesian product $G_{+}(A) \times \mathbb{A}_{\mathbb{K}}^{0 \mid d_{1}}(A)$. Using the bijection $\phi_{A}^{\circ}$ above we push-forward this canonical $A_{\mathbf{0}^{-}}$manifold structure of $G_{+}(A) \times \mathbb{A}_{\mathbb{K}}^{0 \mid d_{1}}(A)$ onto $G_{\mathcal{P}}^{\circ}(A)$, which then is turned into an $A_{\mathbf{0}^{-}}$manifold on its own, still of the same type as $\mathcal{P}$. Strictly speaking, this structure of $A_{\mathbf{0}}$-manifold on $G_{\mathcal{P}}^{\circ}(A)$ formally depends on the choice of $G_{-}^{<}$, hence of a totally ordered $\mathbb{K}$-basis of $\mathfrak{g}_{1}$, as this choice enters in the construction of $\phi_{A}^{\circ}$. However, thanks to the special form of the defining relations of $G_{\mathcal{P}}^{\circ}(A)$ it is straightforward to show that changing such a basis amounts to changing local charts for the same, unique $A_{\mathbf{0}}$-manifold structure; hence the structure is independent of such a choice.

Now, using the above structure of $A_{\mathbf{0}^{-}}$manifold on $G_{\mathcal{P}}^{\circ}(A)$ for each $A \in(\mathbf{W} \text { salg })_{\mathbb{K}}$, given a morphism $f$ : $A^{\prime} \longrightarrow A^{\prime \prime}$ in $(\mathbf{W} \text { salg })_{\mathbb{K}}$ it is straightforward to check that the group morphism $G_{\mathcal{P}}^{\circ}(f): G_{\mathcal{P}}^{\circ}\left(A^{\prime}\right) \longrightarrow G_{\mathcal{P}}^{\circ}\left(A^{\prime \prime}\right)$ is a morphism of $A_{\mathbf{0}}^{\prime}$-manifolds, hence it is a morphism of $\mathcal{A}_{\mathbf{0}}$-manifolds (cf. [3]). Thus $G_{\mathcal{P}}^{\circ}$ is also a functor from Weil $\mathbb{K}$-superalgebras to $\mathcal{A}_{\mathbf{0}}$-manifolds (real smooth, real analytic or complex holomorphic as $\mathcal{P}$ is).

At last, again looking at the commutation relations in $G_{\mathcal{P}}^{\circ}(A)$, we see that the group multiplication and the inverse map are "regular" (i.e., "real smooth", "real analytic" or "complex holomorphic" depending on the type of $\mathcal{P}$ ); indeed, this is explicitly proved by calculations like those needed in the proof of Proposition 
3.2.1(b) - that we skipped, so refer instead to the proof of Proposition 2.4.8(b). Thus they are morphisms of $\mathcal{A}_{\mathbf{0}}$-manifolds, so $G_{\mathcal{P}}^{\circ}(A)$ is a group element among $\mathcal{A}_{\mathbf{0}}$-manifolds, i.e. it is a Lie $\mathcal{A}_{\mathbf{0}}$-group; hence (cf. $\S 2.1 .3)$ the functor $G_{\mathcal{P}}^{\circ}$ is a Lie supergroup (of real smooth, real analytic or complex holomorphic type as $\mathcal{P}$ ).

Eventually, the outcome of this discussion — and core result of the present section — is the following statement, which provides a "backward functor" from sHCp's to Lie supergroups:

Theorem 3.2.6. The recipe in Definition 3.1.1 provides functors

$$
\Psi^{\circ}:(\mathbf{s H C p})_{\mathbb{R}}^{\infty} \longrightarrow(\text { Lsgrp })_{\mathbb{R}}^{\infty} \quad, \quad \Psi^{\circ}:(\mathbf{s H C p})_{\mathbb{R}}^{\omega} \longrightarrow(\text { Lsgrp })_{\mathbb{R}}^{\omega}, \quad \Psi^{\circ}:(\mathbf{s H C p})_{\mathbb{C}}^{\omega} \longrightarrow(\text { Lsgrp })_{\mathbb{C}}^{\omega}
$$

given on objects by $\mathcal{P} \mapsto \Psi^{\circ}(\mathcal{P}):=G_{\mathcal{P}}^{\circ}$ and on morphisms by

$$
\left(\left(\phi_{+}, \varphi\right): \mathcal{P}^{\prime} \longrightarrow \mathcal{P}^{\prime \prime}\right) \mapsto\left(\Psi^{\circ}\left(\left(\phi_{+}, \varphi\right)\right): \Psi^{\circ}\left(\mathcal{P}^{\prime}\right):=G_{\mathcal{P}^{\prime}}^{\circ} \longrightarrow G_{\mathcal{P}^{\prime \prime}}^{\circ}=: \Psi^{\circ}\left(\mathcal{P}^{\prime \prime}\right)\right)
$$

where the functor morphism $\Psi^{\circ}\left(\left(\phi_{+}, \varphi\right)\right): \Psi^{\circ}\left(\mathcal{P}^{\prime}\right):=G_{\mathcal{P}^{\prime}}^{\circ} \longrightarrow G_{\mathcal{P}^{\prime \prime}}^{\circ}=: \Psi^{\circ}\left(\mathcal{P}^{\prime \prime}\right)$ is defined by

$$
\Psi^{\circ}\left(\left(\phi_{+}, \varphi\right)\right)_{A} \quad: \quad g_{+}^{\prime} \mapsto \phi_{+}\left(g_{+}^{\prime}\right), \quad\left(1+\eta Y^{\prime}\right) \mapsto\left(1+\eta \varphi\left(Y^{\prime}\right)\right)
$$

for all $A \in(\mathbf{W s a l g})_{\mathbb{K}}, g_{+}^{\prime} \in G_{+}^{\prime}(A), \eta \in A_{\mathbf{1}}, Y^{\prime} \in \mathfrak{g}^{\prime}$, with $\mathcal{P}^{\prime}=\left(G_{+}^{\prime}, \mathfrak{g}^{\prime}\right)$ and $\mathcal{P}^{\prime \prime}=\left(G_{+}^{\prime \prime}, \mathfrak{g}^{\prime \prime}\right)$.

Proof. What is still left to prove is that the given definition for $\Psi^{\circ}\left(\left(\phi_{+}, \varphi\right)\right)$ actually makes sense. Now, (3.6) above fixes the values of our would-be morphism $\Psi^{\circ}\left(\left(\phi_{+}, \varphi\right)\right)_{A}$ on generators of $\Psi^{\circ}\left(\mathcal{P}^{\prime}\right)(A):=G_{\mathcal{P}^{\prime}}^{\circ}(A)$ : a direct check shows that all defining relations among such generators are respected, thus providing a group morphism as required. However, we must still show that this is a morphism of $\mathcal{A}_{\mathbf{0}}$-manifolds too.

Let $\left\{Y_{i}^{\prime}\right\}_{i \in I}$ and $\left\{Y_{j}^{\prime \prime}\right\}_{j \in J}$ be totally ordered $\mathbb{K}$-bases of $\mathfrak{g}_{1}^{\prime}$ and $\mathfrak{g}_{\mathbf{1}}^{\prime \prime}$. Accordingly, both $G_{\mathcal{P}^{\prime}}^{\circ}(A)$ and $G_{\mathcal{P}^{\prime \prime}}^{\circ}(A)$ admit factorizations as in Proposition 3.2.4(a), hence any $g^{\prime} \in G_{\mathcal{P}^{\prime}}^{\circ}(A)$ uniquely factors into $g^{\prime}=$ $g_{+}^{\prime} \cdot \overrightarrow{\prod_{i \in I}}\left(1+\eta_{i} Y_{i}^{\prime}\right)$; then $\Psi^{\circ}\left(\left(\phi_{+}, \varphi\right)\right)_{A}\left(g^{\prime}\right)=\phi_{+}\left(g_{+}^{\prime}\right) \cdot \vec{\prod}_{i \in I}\left(1+\eta_{i} \varphi\left(Y_{i}^{\prime}\right)\right)$ and from this, letting $\varphi\left(Y_{i}^{\prime}\right)=\sum_{j \in J} c_{i, j} Z_{j}$ - with $c_{i, j} \in \mathbb{K}$ - we get $\Psi^{\circ}\left(\left(\phi_{+}, \varphi\right)\right)_{A}\left(g^{\prime}\right)=\phi_{+}\left(g_{+}^{\prime}\right) \cdot \prod_{i \in I} \prod_{j \in J}\left(1+\eta_{i} c_{i, j} Z_{j}\right)$. Now we must re-order the result according to the factorization of $G_{\mathcal{P}^{\prime \prime}}^{\circ}(A)$ of the form $G_{+} \times G_{-}^{<}$; in doing this, when we reorder the factor $\prod_{i \in I} \prod_{j \in J}\left(1+\eta_{i} c_{i, j} Z_{j}\right)$ we find, via calculations as for Proposition 2.4.8(b), an outcome of the form $\prod_{r=1}^{n}\left(1+a_{r} X_{r}\right)_{G_{+}^{\prime \prime}} \cdot \overrightarrow{\prod_{j \in J}}\left(1+\alpha_{j} Z_{j}\right)$ where the $X_{r}$ 's belong to $\mathfrak{g}_{\mathbf{0}}^{\prime \prime}$, the $a_{r}$ 's are (even) polynomial expressions in the $\eta_{i}$ 's, the $\alpha_{j}$ 's are (odd) polynomial expressions in the $\eta_{i}$ 's. This implies that the map $\vec{\prod}_{i \in I}\left(1+\eta_{i} Y_{i}^{\prime}\right) \mapsto \prod_{r=1}^{n}\left(1+a_{r} X_{r}\right)_{G_{+}^{\prime \prime}} \cdot \vec{\prod}_{j \in J}\left(1+\alpha_{j} Z_{j}\right)$ is a map of $\mathcal{A}_{\mathbf{0}^{-}}$manifolds from $\left(G_{\mathcal{P}^{\prime}}^{\circ}\right)_{-}^{<}(A)$ to $G_{\mathcal{P}^{\prime \prime}}^{\circ}(A)$. But $\phi_{+}: G_{+}^{\prime}(A) \stackrel{r=1}{\longrightarrow} G_{+}^{\prime \prime}(A)$ is a map of $\mathcal{A}_{\mathbf{0}^{-}}$-manifolds too, by assumptions; this along with all the previous analysis eventually implies that $\Psi^{\circ}\left(\left(\phi_{+}, \varphi\right)\right)_{A}$ is a map of $\mathcal{A}_{\mathbf{0}^{-}}$-manifolds as claimed.

\subsection{Supergroup functors out of super Harish-Chandra pairs: second recipe}

In this subsection we construct a second functor, denoted $G_{\mathcal{p}}^{e}$, which we later prove is a Lie supergroup: this is in fact a "sibling alternative" to the functor $G_{\mathcal{P}}^{\circ}$ considered in $\S 3.1$ above. As a matter of notation, recall that for any $\mathcal{P}=\left(G_{+}, \mathfrak{g}\right) \in(\mathbf{s H C p})_{\mathbb{K}}, A \in(\mathbf{W} \mathbf{s a l g})_{\mathbb{K}}$ and $\mathcal{X} \in A_{\mathbf{0}} \otimes_{\mathbb{K}} \mathfrak{g}_{\mathbf{0}}$ there exists a well-defined $\exp _{G_{+}}(\mathcal{X}) \in G_{+}\left(A_{\mathbf{0}}\right)$; furthermore, if in particular $\mathcal{X} \in A_{\mathbf{1}}^{2} \otimes_{\mathbb{K}} \mathfrak{g}_{\mathbf{0}}$, then the formal series expansion of $\exp (\mathcal{X})$ can be actually realized as a finite sum. When no confusion is possible we shall drop the subscript $G_{+}$and simply write $\exp (\mathcal{X})$ instead. Similarly, we shall presently introduce new formal elements of type " $\exp (\mathcal{Y}) "$ with $\mathcal{Y} \in A_{\mathbf{1}} \otimes_{\mathbb{K}} \mathfrak{g}_{\mathbf{1}}$. Finally, we extend the built-in $G_{+}$-action onto $\mathfrak{g}_{\mathbf{1}}$ to a (same-name) $G_{+}-$action onto $A_{\mathbf{1}} \otimes_{\mathbb{K}} \mathfrak{g}_{\mathbf{1}}$ by $\operatorname{Ad}(g)\left(\sum_{s=1}^{n} \eta_{s} Y_{s}\right):=\sum_{s=1}^{n} \eta_{s} A d(g)\left(Y_{s}\right)$ for all $\sum_{s=1}^{n} \eta_{s} Y_{s}:=\sum_{s=1}^{n} \eta_{s} \otimes Y_{s} \in A_{\mathbf{1}} \otimes_{\mathbb{K}} \mathfrak{g}_{\mathbf{1}}$.

Definition 3.3.1. Let $\mathcal{P}:=\left(G_{+}, \mathfrak{g}\right) \in(\mathbf{s H C p})_{\mathbb{K}}$ be a super Harish-Chandra pair over $\mathbb{K}$.

(a) We introduce a functor $G_{\mathcal{P}}^{e}:(\text { Wsalg })_{\mathbb{K}} \longrightarrow$ (group) as follows. For any Weil superalgebra $A \in(\mathbf{W} \mathbf{s a l g})_{\mathbb{K}}$, we define $G_{\mathcal{P}}^{e}(A)$ as being the group with generators the elements of the set 


$$
\Gamma_{A}:=\left\{g_{+}, \exp (\mathcal{Y}) \mid g_{+} \in G_{+}(A), \mathcal{Y} \in A_{\mathbf{1}} \otimes_{\mathbb{K}} \mathfrak{g}_{\mathbf{1}}\right\}=G_{+}(A) \bigcup\{\exp (\mathcal{Y})\}_{\mathcal{Y} \in A_{1} \otimes_{\mathbb{K}} \mathfrak{g}_{1}}
$$

and relations (for $g_{+}, g_{+}^{\prime}, g_{+}^{\prime \prime} \in G_{+}(A), \mathcal{Y}, \mathcal{Y}^{\prime}, \mathcal{Y}^{\prime \prime} \in A_{1} \otimes_{\mathbb{K}} \mathfrak{g}_{1}$ )

$$
\begin{gathered}
g_{+}^{\prime} \cdot g_{+}^{\prime \prime}=g_{+\dot{a}_{+}}^{\prime} g_{+}^{\prime \prime}, \quad \exp (0)=1, \quad \exp (\mathcal{Y}) \cdot g_{+}=g_{+} \cdot \exp \left(\operatorname{Ad}\left(g_{+}^{-1}\right)(\mathcal{Y})\right) \\
\exp \left(\mathcal{Y}^{\prime}\right) \cdot \exp \left(\mathcal{Y}^{\prime \prime}\right)=\exp \left(P_{\mathbf{0}}^{\left(d_{1}\right)}\left(\mathcal{Y}^{\prime}, \mathcal{Y}^{\prime \prime}\right)\right) \cdot \exp \left(\mathcal{Y}^{\prime}+\mathcal{Y}^{\prime \prime}+P_{\mathbf{1}}^{\left(d_{1}\right)}\left(\mathcal{Y}^{\prime}, \mathcal{Y}^{\prime \prime}\right)\right)
\end{gathered}
$$

with $P_{\mathbf{0}}^{\left(d_{1}\right)}$ and $P_{\mathbf{1}}^{\left(d_{1}\right)}$ as given in Lemma 2.4.5 $(h)$. This yields the functor $G_{\mathcal{P}}^{e}$ on objects.

To define $G_{\mathcal{P}}^{e}$ on morphisms, for any morphism $f: A^{\prime} \longrightarrow A^{\prime \prime}$ in $(\mathbf{W s a l g})_{\mathbb{K}}$ we define the group morphism $G_{\mathcal{P}}^{e}(f): G_{\mathcal{P}}^{e}\left(A^{\prime}\right) \longrightarrow G_{\mathcal{P}}^{e}\left(A^{\prime \prime}\right)$ to be the unique one given - for $g_{+}^{\prime} \in G_{+}\left(A^{\prime}\right), \eta \in A_{\mathbf{1}}^{\prime}, \mathcal{Y}^{\prime} \in A_{\mathbf{1}}^{\prime} \otimes_{\mathbb{K}} \mathfrak{g}_{\mathbf{1}}$ - by

$$
G_{\mathcal{P}}^{e}(f)\left(g_{+}^{\prime}\right):=G_{+}(f)\left(g_{+}^{\prime}\right) \quad, \quad G_{\mathcal{P}}^{e}(f)(\exp (\mathcal{Y})):=\exp (f(\mathcal{Y}))
$$

where $f\left(\mathcal{Y}^{\prime}\right):=\sum_{s=1}^{n} f\left(\eta_{s}^{\prime}\right) Y_{s}$ for all $\mathcal{Y}^{\prime}:=\sum_{s=1}^{n} \eta_{s}^{\prime} Y_{s} \in A_{\mathbf{1}}^{\prime} \otimes_{\mathbb{K}} \mathfrak{g}_{\mathbf{1}}$.

(b) We define a functor $G_{\mathcal{P}}^{e,-}:(\mathbf{W} \text { salg })_{\mathbb{K}} \longrightarrow($ set $)$ on any object $A \in(\text { Wsalg })_{\mathbb{K}}$ by

$$
G_{\mathcal{P}}^{e,-}(A):=\left\langle\exp \left(A_{1} \otimes_{\mathbb{K}} \mathfrak{g}_{1}\right)\right\rangle \quad\left(\subseteq G_{\mathcal{P}}^{e}(A)\right)
$$

— the subgroup of $G_{\mathcal{P}}^{e}(A)$ generated by $\exp \left(A_{\mathbf{1}} \otimes_{\mathbb{K}} \mathfrak{g}_{1}\right):=\{\exp (\mathcal{Y})\}_{\mathcal{Y} \in A_{1} \otimes_{\mathbb{K}} \mathfrak{g}_{1}}$ - and on morphisms in the obvious way. By definition, $G_{\mathcal{P}}^{e,-}$ can be thought of as subfunctor of $G_{\mathcal{P}}^{e}$.

3.3.2. Another realization of $G_{\mathcal{P}}^{e}$. Given a super Harish-Chandra pair $\mathcal{P}=\left(G_{+}, \mathfrak{g}\right) \in(\mathbf{s H C p})_{\mathbb{K}}$, we present now another way of realizing the $\mathbb{K}$-supergroup $G_{\mathcal{p}}^{e}$ introduced in Definition 3.3.1(a): this mimics what we did in $§ 3.1 .4$, so we keep the same kind of notation and are a bit shorter.

For any fixed $A \in(\mathbf{W} \text { salg })_{\mathbb{K}}$, we denote by $G_{+}^{\langle 2\rangle}(A)$ the subgroup of $G_{+}(A)$ generated by the set $\left\{\exp (\mathcal{X}) \mid \mathcal{X} \in A_{1}^{[2]} \otimes_{\mathbb{K}}\left[\mathfrak{g}_{1}, \mathfrak{g}_{1}\right]\right\}$. Then one easily sees that $G_{+}^{\langle 2\rangle}(A)$ is normal in $G_{+}(A)$. Consider also

$$
\Gamma_{A}^{+}:=G_{+}(A), \quad \Gamma_{A}^{\langle 2\rangle}:=G_{+}^{\langle 2\rangle}(A), \quad \Gamma_{A}^{-}:=\Gamma_{A}^{\langle 2\rangle} \bigcup \exp \left(A_{\mathbf{1}} \otimes_{\mathbb{K}} \mathfrak{g}_{\mathbf{1}}\right)
$$

and the five sets of relations (for all $g_{+}, g_{+}^{\prime}, g_{+}^{\prime \prime} \in \Gamma_{A}^{+}, g_{\langle 2\rangle}, g_{\langle 2\rangle}^{\prime}, g_{\langle 2\rangle}^{\prime \prime} \in \Gamma_{A}^{\langle 2\rangle}, \mathcal{Y}, \mathcal{Y}^{\prime}, \mathcal{Y}^{\prime \prime} \in A_{1} \otimes_{\mathbb{K}} \mathfrak{g}_{1}$ )

$$
\begin{gathered}
\mathcal{R}_{A}^{+}: g_{+}^{\prime} \cdot g_{+}^{\prime \prime}=g_{+\dot{G}_{+}}^{\prime} g_{+}^{\prime \prime}, \quad \mathcal{R}_{A}^{\langle 2\rangle}: \quad\left(g_{\langle 2\rangle}\right)_{\Gamma_{A}^{\langle 2\rangle}}=\left(g_{\langle 2\rangle}\right)_{\Gamma_{A}^{+}} \\
\mathcal{R}_{A}^{\ltimes}: \quad g_{\langle 2\rangle} \cdot g_{+}=g_{+} \cdot\left(g_{+}^{-1} \dot{G}_{+} g_{\langle 2\rangle \dot{G}_{+}} g_{+}\right), \quad \exp (\mathcal{Y}) \cdot g_{+}=g_{+} \cdot \exp \left(\operatorname{Ad}\left(g_{+}^{-1}\right)(\mathcal{Y})\right) \\
\mathcal{R}_{A}^{-}:\left\{\begin{array}{c}
g_{\langle 2\rangle}^{\prime} \cdot g_{\langle 2\rangle}^{\prime \prime}=g_{\langle 2\rangle}^{\prime} \dot{G}_{+}^{\langle 2\rangle} g_{\langle 2\rangle}^{\prime \prime}, \quad \exp (\mathcal{Y}) \cdot g_{\langle 2\rangle}=g_{\langle 2\rangle} \cdot \exp \left(\operatorname{Ad}\left(g_{\langle 2\rangle}^{-1}\right)(\mathcal{Y})\right), \quad \exp (0)=1 \\
\exp \left(\mathcal{Y}^{\prime}\right) \exp \left(\mathcal{Y}^{\prime \prime}\right)=\exp \left(P_{\mathbf{0}}^{\left(d_{1}\right)}\left(\mathcal{Y}^{\prime}, \mathcal{Y}^{\prime \prime}\right)\right) \exp \left(\mathcal{Y}^{\prime}+\mathcal{Y}^{\prime \prime}+P_{\mathbf{1}}^{\left(d_{1}\right)}\left(\mathcal{Y}^{\prime}, \mathcal{Y}^{\prime \prime}\right)\right) \\
\text { with } P_{\mathbf{0}}^{\left(d_{1}\right)} \text { and } P_{\mathbf{1}}^{\left(d_{1}\right)} \text { as given in Lemma 2.4.5 }(h) \\
\mathcal{R}_{A}:=\mathcal{R}_{A}^{+} \cup \mathcal{R}_{A}^{-} \cup \mathcal{R}_{A}^{\ltimes} \cup \mathcal{R}_{A}^{\langle 2\rangle}
\end{array}\right.
\end{gathered}
$$

Then we define a new group, by generators and relations, namely $G_{\mathcal{P}}^{e,-}(A):=\left\langle\Gamma_{A}^{-}\right\rangle /\left(\mathcal{R}_{A}^{-}\right)$.

Directly from Definition 3.3.1 it follows that

$$
G_{\mathcal{P}}^{e}(A) \cong\left\langle\Gamma_{A}^{+} \bigcup \Gamma_{A}^{-}\right\rangle /\left(\mathcal{R}_{A}\right)=\left\langle\Gamma_{A}^{+} \bigcup \Gamma_{A}^{-}\right\rangle /\left(\mathcal{R}_{A}^{+} \cup \mathcal{R}_{A}^{-} \cup \mathcal{R}_{A}^{\ltimes} \cup \mathcal{R}_{A}^{\langle 2\rangle}\right)
$$

but we can also achieve the presentation (3.7) in a series of intermediate steps. As a first step, we have

$$
\left\langle\Gamma_{A}^{+} \cup \Gamma_{A}^{-}\right\rangle /\left(\mathcal{R}_{A}^{+} \cup \mathcal{R}_{A}^{-}\right)=\left\langle\Gamma_{A}^{+}\right\rangle /\left(\mathcal{R}_{A}^{+}\right) *\left\langle\Gamma_{A}^{-}\right\rangle /\left(\mathcal{R}_{A}^{-}\right) \cong G_{+}(A) * G_{\mathcal{P}}^{e,-}(A)
$$


where $G_{+}(A) \cong\left\langle\Gamma_{A}^{+}\right\rangle /\left(\mathcal{R}_{A}^{+}\right)$by construction and $*$ denotes the free product (of two groups).

For the next two steps we can follow two different lines of action. The first one gives

$$
\left\langle\Gamma_{A}^{+} \cup \Gamma_{A}^{-}\right\rangle /\left(\mathcal{R}_{A}^{+} \cup \mathcal{R}_{A}^{-} \cup \mathcal{R}_{A}^{\ltimes}\right) \cong\left(G_{+}(A) * G_{\mathcal{P}}^{e,-}(A)\right) /\left(\overline{\mathcal{R}_{A}^{\ltimes}}\right) \cong G_{+}(A) \ltimes G_{\mathcal{P}}^{e,-}(A)
$$

because of (3.1) and (3.8), where $G_{+}(A) \ltimes G_{\mathcal{P}}^{e,-}(A)$ is the semidirect product of $G_{+}(A)$ with $G_{\mathcal{P}}^{e,-}(A)$. Then $\left\langle\Gamma_{A}^{+} \cup \Gamma_{A}^{-}\right\rangle /\left(\mathcal{R}_{A}\right) \cong\left\langle\Gamma_{A}^{+} \cup \Gamma_{A}^{-}\right\rangle /\left(\mathcal{R}_{A}^{+} \cup \mathcal{R}_{A}^{-} \cup \mathcal{R}_{A}^{\ltimes} \cup \mathcal{R}_{A}^{\langle 2\rangle}\right) \cong\left(G_{+}(A) \ltimes G_{\mathcal{P}}^{\circ,-}(A)\right) /\left(\overline{\mathcal{R}_{A}^{\langle 2\rangle}}\right) \cong$ $\cong\left(G_{+}(A) \ltimes G_{\mathcal{P}}^{\circ,-}(A)\right) / N_{\langle 2\rangle}(A)$, where $N_{\langle 2\rangle}(A)$ is the normal subgroup of $G_{+}(A) \ltimes G_{\mathcal{P}}^{e,-}(A)$ generated by $\left\{\left(g_{\langle 2\rangle}, g_{\langle 2\rangle}^{-1}\right)\right\}_{g_{\langle 2\rangle} \in \Gamma_{A}^{\langle 2\rangle}}$. This and (3.7) together yield $G_{\mathcal{P}}^{e}(A)=\left(G_{+}(A) \ltimes G_{-}(A)\right) / N_{\langle 2\rangle}(A)$.

On the other hand, (3.1) and (3.8) jointly give

$$
\left\langle\Gamma_{A}^{+} \cup \Gamma_{A}^{-}\right\rangle /\left(\mathcal{R}_{A}^{+} \cup \mathcal{R}_{A}^{-} \cup \mathcal{R}_{A}^{\langle 2\rangle}\right) \cong\left(G_{+}(A) * G_{\mathcal{P}}^{e,-}(A)\right) /\left(\overline{\mathcal{R}_{A}^{\langle 2\rangle}}\right) \cong G_{+}(A)_{G_{+}^{\langle 2\rangle}(A)}^{*} G_{\mathcal{P}}^{e,-}(A)
$$

with $G_{+}(A) \underset{G_{+}^{\langle 2\rangle}(A)}{*} G_{\mathcal{P}}^{e,-}(A)$ the amalgamated product of $G_{+}(A)$ and $G_{\mathcal{P}}^{e,-}(A)$ over $G_{+}^{\langle 2\rangle}(A)$. Then

$$
\begin{aligned}
\left\langle\Gamma_{A}^{+} \cup \Gamma_{A}^{-}\right\rangle /\left(\mathcal{R}_{A}\right) \cong & \left\langle\Gamma_{A}^{+} \cup \Gamma_{A}^{-}\right\rangle /\left(\mathcal{R}_{A}^{+} \cup \mathcal{R}_{A}^{-} \cup \mathcal{R}_{A}^{\langle 2\rangle} \cup \mathcal{R}_{A}^{\ltimes}\right) \cong \\
& \cong\left(G_{+}(A) \underset{G_{+}^{\langle 2\rangle}(A)}{*} G_{\mathcal{P}}^{e,-}(A)\right) /\left(\overline{\mathcal{R}_{A}^{\ltimes}}\right) \cong\left(G_{+}(A)_{G_{+}^{\langle 2\rangle}(A)}^{*} G_{\mathcal{P}}^{e,-}(A)\right) / N_{\ltimes}(A)
\end{aligned}
$$

where $N_{\ltimes}(A)$ is the normal subgroup of $G_{+}(A) \underset{G_{+}^{\langle 2\rangle}(A)}{*} G_{\mathcal{P}}^{e,-}(A)$ generated by

$$
\left\{g_{+} \exp (\mathcal{Y}) g_{+}^{-1} \exp \left(A d\left(g_{+}\right)(\mathcal{Y})\right)^{-1}\right\}_{\mathcal{Y} \in A_{1} \otimes_{\mathbb{K}} \mathfrak{g}_{1}}^{g_{+} \in G_{+}(A)} \cup\left\{g_{+} g_{\langle 2\rangle} g_{+}\left(g_{+} \dot{G}_{+} g_{\langle 2\rangle_{G_{+}}} g_{+}\right)^{-1}\right\}_{g_{+} \in G_{+}(A)}^{g_{\langle 2} \in \Gamma_{A}^{\langle 2\rangle}}
$$

All this along with (3.7) eventually gives $G_{\mathcal{P}}^{e}(A)=\left(G_{+}(A) \underset{G_{+}^{\langle 2\rangle}(A)}{*} G_{\mathcal{P}}^{e,-}(A)\right) / N_{\ltimes}(A)$ for every $A \in$ $(\mathbf{W} \text { salg })_{\mathbb{K}}$. In functorial terms this means $G_{\mathcal{P}}^{e}=\left(G_{+} \ltimes G_{\mathcal{P}}^{e,-}\right) / N_{\langle 2\rangle}$ and $G_{\mathcal{P}}^{e}=\left(G_{+} G_{+}^{2 \lambda} G_{\mathcal{P}}^{e,-}\right) / N_{\ltimes}$, or $G_{\mathcal{P}}^{e}=G_{+} \underset{G_{+}^{\langle 2\rangle}}{\times} G_{\mathcal{P}}^{e,-}$ so that $G_{\mathcal{P}}^{e}$ is the "amalgamate semidirect product" of $G_{+}$and $G_{\mathcal{P}}^{e,-}$ over $G_{+}^{\langle 2\rangle}$.

\subsection{The supergroup functor $G_{\mathcal{P}}^{e}$ as a Lie supergroup}

We aim now to prove that the functor $G_{\mathcal{P}}^{e}$ is actually a Lie supergroup. We follow in the footsteps of in $\S 3.2$, so we can be somewhat shorter. We begin with the following "factorization result" for $G_{\mathcal{P}}^{e}$ :

Proposition 3.4.1. Let $\mathcal{P}:=\left(G_{+}, \mathfrak{g}\right) \in(\mathbf{s H C p})_{\mathbb{K}}$ be a super Harish-Chandra pair over $\mathbb{K}$. Then there exist group-theoretic factorizations

$$
\begin{array}{rll}
G_{\mathcal{P}}^{e,-}(A)=G_{+}^{\langle 2\rangle}(A) \cdot \exp \left(A_{\mathbf{1}} \otimes_{\mathbb{K}} \mathfrak{g}_{1}\right) & , \quad & G_{\mathcal{P}}^{e,-}(A)=\exp \left(A_{\mathbf{1}} \otimes_{\mathbb{K}} \mathfrak{g}_{\mathbf{1}}\right) \cdot G_{+}^{\langle 2\rangle}(A) \\
G_{\mathcal{P}}^{e}(A)=G_{+}(A) \cdot \exp \left(A_{\mathbf{1}} \otimes_{\mathbb{K}} \mathfrak{g}_{\mathbf{1}}\right) \quad, \quad & G_{\mathcal{P}}^{e}(A)=\exp \left(A_{\mathbf{1}} \otimes_{\mathbb{K}} \mathfrak{g}_{\mathbf{1}}\right) \cdot G_{+}(A)
\end{array}
$$

Proof. Claim (a) is the exact analogue of (2.8), and claim (b) the analogue of (2.10), in Proposition 2.4.8(c). In both cases the proof (up to details) is the same, so we can skip it. 
3.4.2. The representation $G_{\mathcal{P}}^{e} \longrightarrow \mathrm{GL}(V)$. Let $\mathcal{P}=\left(G_{+}, \mathfrak{g}\right) \in(\mathbf{s H C p})_{\mathbb{K}}$ be any given super HarishCandra pair over $\mathbb{K}$. Just like we $\operatorname{did}$ for $G_{\mathcal{P}}^{\circ}$ in $\S 3.2 .2$, we need for $G_{\mathcal{p}}^{e}$ as well a suitable linear representation $V$, which we now define along the same lines, keeping the same notation.

Let $U(\mathfrak{g})$ be the universal enveloping algebra of $\mathfrak{g}$, and let $V:=\operatorname{Ind} d_{\mathfrak{g}_{0}}^{\mathfrak{g}}(\mathbf{1})=U(\mathfrak{g}) \underset{U\left(\mathfrak{g}_{0}\right)}{\otimes} \mathbb{1} \cong \bigwedge \mathfrak{g}_{1}$ be the $\mathfrak{g}$-representation induced from the trivial representation $\mathbf{1}$ of $\mathfrak{g}_{\mathbf{0}}$ - as in (3.5). As we saw in §3.2.2, there is a morphism $\left(\boldsymbol{r}_{+}, \rho\right):\left(G_{+} \mathfrak{g}\right) \longrightarrow(\mathrm{GL}(V), \mathfrak{g l}(V))$ of sHCp's making $V$ into a $\left(G_{+}, \mathfrak{g}\right)$-module; again by $\rho$ we denote also the representation map $\rho: U(\mathfrak{g}) \longrightarrow \operatorname{End}_{\mathbb{K}}(V)$ giving the $U(\mathfrak{g})$-module structure on $V$, and similarly (in a functorial way) for the representation maps of $\left(A \otimes_{\mathbb{K}} \mathfrak{g}\right)_{\mathbf{0}}$ and $\left(A \otimes_{\mathbb{K}} U(\mathfrak{g})\right)_{\mathbf{0}}$ onto $\left(A \otimes_{\mathbb{K}} V\right)_{\mathbf{0}}$.

We will now show that the $\left(G_{+}, \mathfrak{g}\right)$-module structure on $V$ can be "integrated" to a $G_{\mathcal{P}}^{e}$-module structure.

Proposition 3.4.3. Retain notation as above for the $\left(G_{+}, \mathfrak{g}\right)$-module $V$. There exists a unique structure of (left) $G_{p}^{e}$-module onto $V$ which satisfies the following conditions: for every $A \in(\mathbf{W} \text { salg })_{\mathbb{K}}$, the representation map $\boldsymbol{r}_{\mathcal{P}, A}^{e}: G_{\mathcal{P}}^{e}(A) \longrightarrow \mathrm{GL}(V)(A)$ is given on generators of $G_{\mathcal{P}}^{e}(A)-$ namely, all $g_{+} \in G_{+}(A)$ and $\exp (\mathcal{Y})$ for $i \in I, \mathcal{Y} \in A_{\mathbf{1}} \otimes_{\mathbb{K}} \mathfrak{g}_{\mathbf{1}}-$ by $\boldsymbol{r}_{\mathcal{P}, A}^{e}\left(g_{+}\right):=\boldsymbol{r}_{+}\left(g_{+}\right), \quad \boldsymbol{r}_{\mathcal{P}, A}^{e}(\exp (\mathcal{Y})):=\rho(\exp (\mathcal{Y}))=\exp (\rho(\mathcal{Y}))$, that is $g_{+} \cdot v:=\boldsymbol{r}_{+}\left(g_{+}\right)(v)$ and $\exp (\mathcal{Y}) \cdot v:=\exp (\rho(\mathcal{Y}))(v)$ - with $\exp (\rho(\mathcal{Y}))$ being a finite sum - for all $v \in V(A)$. Overall, this yields a morphism of $\mathbb{K}$-supergroup functors $\boldsymbol{r}_{\mathcal{p}}^{e}: G_{\mathcal{P}}^{e} \longrightarrow \mathrm{GL}(V)$.

Proof. By definition of representation for the sHCp $\mathcal{P}$, the operators $\boldsymbol{r}_{\mathcal{P}, A}^{e}\left(g_{+}\right)$and $\boldsymbol{r}_{\mathcal{P}_{A}}^{e}(\exp (\mathcal{Y}))$ on $V$ satisfy all relations which, by Definition 3.3.1 $(a)$, are satisfied by the generators of $G_{\mathcal{P}}^{e}(A)$. Thus they define a unique group morphism $\boldsymbol{r}_{\mathcal{P}, A}^{e}: G_{\mathcal{P}}^{e}(A) \longrightarrow \mathrm{GL}(V)(A)$, functorial in $A$ by construction, whence the claim.

We are now ready to state the "global splitting theorem" for $G_{\mathcal{P}}^{e}$ (cf. Theorem 2.4.10):

\section{Proposition 3.4.4.}

(a) The restriction of group multiplication in $G_{\mathcal{P}}^{e}$ provides isomorphisms of (set-valued) functors

$$
\begin{aligned}
& G_{+} \times \exp \left((-)_{1} \otimes_{\mathbb{K}} \mathfrak{g}_{1}\right) \cong G_{\mathcal{P}}^{e} \quad, \quad \exp \left((-)_{1} \otimes_{\mathbb{K}} \mathfrak{g}_{1}\right) \times G_{+} \cong G_{\mathcal{P}}^{e} \\
& G_{+}^{\langle 2\rangle} \times \exp \left((-)_{\mathbf{1}} \otimes_{\mathbb{K}} \mathfrak{g}_{\mathbf{1}}\right) \cong G_{\mathcal{P}}^{e,-} \quad, \quad \exp \left((-)_{\mathbf{1}} \otimes_{\mathbb{K}} \mathfrak{g}_{\mathbf{1}}\right) \times G_{+}^{\langle 2\rangle} \cong G_{\mathcal{P}}^{e,-}
\end{aligned}
$$

(b) For any fixed $\mathbb{K}$-basis $\left\{Y_{i}\right\}_{i \in I}$ of $\mathfrak{g}_{1}$, there exists an isomorphism of (set-valued) functors $\mathbb{A}_{\mathbb{K}}^{0 \mid d_{1}} \cong$ $\exp \left((-)_{1} \otimes_{\mathbb{K}} \mathfrak{g}_{1}\right)$, with $d_{1}:=\operatorname{dim}_{\mathbb{K}}\left(\mathfrak{g}_{1}\right)=|I|$, given on $A$-points, for $A \in(\mathbf{W s a l g})_{\mathbb{K}}$, by

$$
\mathbb{A}_{\mathbb{K}}^{0 \mid d_{1}}(A)=A_{\mathbf{1}}^{d_{1}} \longrightarrow \exp \left(A_{\mathbf{1}} \otimes_{\mathbb{K}} \mathfrak{g}_{\mathbf{1}}\right), \quad\left(\eta_{i}\right)_{i \in I} \mapsto \exp \left(\sum_{i \in I} \eta_{i} Y_{i}\right)
$$

(c) There exist isomorphisms of (set-valued) functors

$$
G_{+} \times \mathbb{A}_{\mathbb{K}}^{0 \mid d_{1}} \cong G_{\mathcal{P}}^{e}, \quad G_{+}^{\langle 2\rangle} \times \mathbb{A}_{\mathbb{K}}^{0 \mid d_{1}} \cong G_{\mathcal{P}}^{e,-}, \quad \text { and } \quad \mathbb{A}_{\mathbb{K}}^{0 \mid d_{1}} \times G_{+} \cong G_{\mathcal{P}}^{e}, \quad \mathbb{A}_{\mathbb{K}}^{0 \mid d_{1}} \times G_{+}^{\langle 2\rangle} \cong G_{\mathcal{P}}^{e,-}
$$

given on $A$-points - for every $A \in(\mathbf{W} \text { salg })_{\mathbb{K}}$ - respectively by

$$
\left(g_{+},\left(\eta_{i}\right)_{i \in I}\right) \mapsto g_{+} \cdot \exp \left(\sum_{i \in I} \eta_{i} Y_{i}\right) \quad \text { and } \quad\left(\left(\eta_{i}\right)_{i \in I}, g_{+}\right) \mapsto \exp \left(\sum_{i \in I} \eta_{i} Y_{i}\right) \cdot g_{+}
$$

Proof. Like for Proposition 3.2.4, the proof is very close to (half of) that of Theorem 2.4.10, with a few, technical differences that involve the representation $V$ of $\S 3.4 .2$; the necessary changes can easily be dealt with much like in the proof of Proposition 3.2.4. Details are left to the reader.

3.4.5. The Lie supergroup structure of $G_{\mathcal{P}}^{e}$. For any given $\mathcal{P} \in(\mathbf{s H C p})_{\mathbb{K}}$ and $A \in(\mathbf{W s a l g})_{\mathbb{K}}$, by Proposition 3.4.4(c), we have a particular bijection $\phi_{A}^{e}: G_{+}(A) \times \mathbb{A}_{\mathbb{K}}^{0 \mid d_{1}}(A) \stackrel{\cong}{\cong} G_{\mathcal{P}}^{e}(A)$ whose restriction to $G_{+}(A)$, identified with $G_{+}(A) \times\left\{(0)_{i \in I}\right\}$, is the identity - onto the copy of $G_{+}(A)$ inside $G_{\mathcal{P}}^{e}(A)$.

Now, $G_{+}(A)$ is by definition an $A_{0}$-manifold (cf. [3]), of the same type (real smooth, etc.) as $\mathcal{P}:=$ $\left(G_{+}, \mathfrak{g}\right)$; on the other hand, $\mathbb{A}_{\mathbb{K}}^{0 \mid d_{1}}(A)$ carries canonical structures of $A_{\mathbf{0}}$-manifold of any type (real smooth, etc.), then also of the type of $G_{+}(A)$. So there exists also a canonical "product structure" of $A_{0}$-manifold — of the same type of $\mathcal{P}$ - onto $G_{+}(A) \times \mathbb{A}_{\mathbb{K}}^{0 \mid d_{1}}(A)$. Then we push-forward — through $\phi_{A}^{e}$ - this canonical $A_{0}$-manifold structure of $G_{+}(A) \times \mathbb{A}_{\mathbb{K}}^{0 \mid d_{1}}(A)$ onto $G_{\mathcal{P}}^{e}(A)$, which then is an $A_{\mathbf{0}^{-}}$-manifold on its own. 
Using the above mentioned structure of $A_{\mathbf{0}}$-manifold on $G_{\mathcal{P}}^{e}(A)$ for each $A \in(\mathbf{W} \mathbf{s a l g})_{\mathbb{K}}$, given a morphism $f: A^{\prime} \longrightarrow A^{\prime \prime}$ in $(\mathbf{W s a l g})_{\mathbb{K}}$ we can check that the corresponding group morphism $G_{\mathcal{P}}^{e}(f): G_{\mathcal{P}}^{e}\left(A^{\prime}\right) \longrightarrow G_{\mathcal{p}}^{e}\left(A^{\prime \prime}\right)$ is a morphism of $A_{\mathbf{0}}^{\prime}$-manifolds, hence it is a morphism of $\mathcal{A}_{\mathbf{0}}$-manifolds (cf. [3]). Thus $G_{\mathcal{P}}^{e}$ is also a functor from Weil $\mathbb{K}$-superalgebras to $\mathcal{A}_{\mathbf{0}}$-manifolds (real smooth, real analytic or complex holomorphic as $\mathcal{P}$ is).

Finally, looking at the commutation relations in $G_{\mathcal{P}}^{e}(A)$ we find that the group multiplication and the inverse map are "regular" (i.e., "real smooth", "real analytic" or "complex holomorphic", according to the type of $\mathcal{P}$ ): this is proved via calculations like those used to prove Proposition 3.4.1(b) or Proposition 2.4.8(c). So $G_{\mathcal{P}}^{e}(A)$ is a group element among $\mathcal{A}_{\mathbf{0}}$-manifolds, i.e. it is a Lie $\mathcal{A}_{\mathbf{0}}$-group; thus (cf. §2.1.3), the functor $G_{\mathcal{P}}^{e}$ is a Lie supergroup (of real smooth, real analytic or complex holomorphic type as $\mathcal{P}$ is).

Eventually, the outcome of this discussion - the key result of the present section - is the following statement, which provides a second "backward functor" from sHCp's to Lie supergroups:

Theorem 3.4.6. The recipe in Definition 3.3 .1 provides functors

$$
\Psi^{e}:(\mathbf{s H C p})_{\mathbb{R}}^{\infty} \longrightarrow(\text { Lsgrp })_{\mathbb{R}}^{\infty}, \quad \Psi^{e}:(\mathbf{s H C p})_{\mathbb{R}}^{\omega} \longrightarrow(\text { Lsgrp })_{\mathbb{R}}^{\omega}, \quad \Psi^{e}:(\mathbf{s H C p})_{\mathbb{C}}^{\omega} \longrightarrow(\text { Lsgrp })_{\mathbb{C}}^{\omega}
$$

given on objects by $\mathcal{P} \mapsto \Psi^{e}(\mathcal{P}):=G_{\mathcal{P}}^{e}$ and on morphisms by

$$
\left(\left(\phi_{+}, \varphi\right): \mathcal{P}^{\prime} \longrightarrow \mathcal{P}^{\prime \prime}\right) \mapsto\left(\Psi^{e}\left(\left(\phi_{+}, \varphi\right)\right): \Psi^{e}\left(\mathcal{P}^{\prime}\right):=G_{\mathcal{P}^{\prime}}^{e} \longrightarrow G_{\mathcal{P}^{\prime \prime}}^{e}=: \Psi^{e}\left(\mathcal{P}^{\prime \prime}\right)\right)
$$

where the functor morphism $\Psi^{e}\left(\left(\phi_{+}, \varphi\right)\right): \Psi^{e}\left(\mathcal{P}^{\prime}\right):=G_{\mathcal{P}^{\prime}}^{e} \longrightarrow G_{\mathcal{P}^{\prime \prime}}^{e}=: \Psi^{e}\left(\mathcal{P}^{\prime \prime}\right)$ is defined by

$$
\Psi^{e}\left(\left(\phi_{+}, \varphi\right)\right)_{A}: \quad g_{+}^{\prime} \mapsto \phi_{+}\left(g_{+}^{\prime}\right), \quad \exp \left(\mathcal{Y}^{\prime}\right) \mapsto \exp \left(\varphi\left(\mathcal{Y}^{\prime}\right)\right)
$$

for all $A \in(\mathbf{W} \mathbf{s a l g})_{\mathbb{K}}, g_{+}^{\prime} \in G_{+}^{\prime}(A), \mathcal{Y}^{\prime} \in A_{1} \otimes_{\mathbb{K}} \mathfrak{g}_{1}^{\prime}$, with $\mathcal{P}^{\prime}=\left(G_{+}^{\prime}, \mathfrak{g}^{\prime}\right)$ and $\mathcal{P}^{\prime \prime}=\left(G_{+}^{\prime \prime}, \mathfrak{g}^{\prime \prime}\right)$.

Proof. We are left to prove that the given definition for $\Psi^{e}\left(\left(\phi_{+}, \varphi\right)\right)$ does make sense: all the rest is proved by our previous analysis or is trivial. Now, (3.9) above fixes the values of our would-be morphism $\Psi^{e}\left(\left(\phi_{+}, \varphi\right)\right)_{A}$ on generators of $\Psi^{e}\left(\mathcal{P}^{\prime}\right)(A):=G_{\mathcal{P}^{\prime}}^{e}(A)$ : a direct check shows that all defining relations among such generators - in $G_{\mathcal{P}^{\prime}}^{e}(A)$ - are mapped to corresponding relations in $G_{\mathcal{P}^{\prime \prime}}^{e}(A)$, thus yielding a well-defined group morphism. In particular, this follows from the special properties of the Lie polynomials $P_{\mathbf{0}}^{\left(d_{1}\right)}$ and $P_{\mathbf{1}}^{\left(d_{1}\right)}$ and of their factors/summands $T_{\mathbf{0}}^{(s)}$ and $T_{\mathbf{1}}^{(s)}$ mentioned in Lemma 2.4.5 $(h)$.

However, we must still show that each such $\Psi^{e}\left(\left(\phi_{+}, \varphi\right)\right)_{A}$ is a morphism of $\mathcal{A}_{\mathbf{0}}$-manifolds too.

Both groups $G_{\mathcal{P}^{\prime}}^{e}(A)$ and $G_{\mathcal{P}^{\prime \prime}}^{e}(A)$ admit factorizations of type $G_{+} \times \exp \left(A_{\mathbf{1}} \otimes_{\mathbb{K}} \mathfrak{g}_{\mathbf{1}}\right)$, as in Proposition 3.4.4(a): so any $g^{\prime} \in G_{\mathcal{P}^{\prime}}^{e}(A)$ uniquely factors into $g^{\prime}=g_{+}^{\prime} \cdot \exp \left(\mathcal{Y}^{\prime}\right)$; then $\Psi^{e}\left(\left(\phi_{+}, \varphi\right)\right)_{A}$ maps $g^{\prime}$ onto

$$
\Psi^{e}\left(\left(\phi_{+}, \varphi\right)\right)_{A}\left(g^{\prime}\right)=\Psi^{e}\left(\left(\phi_{+}, \varphi\right)\right)_{A}\left(g_{+}^{\prime} \cdot \exp \left(\mathcal{Y}^{\prime}\right)\right)=\phi_{+}\left(g_{+}^{\prime}\right) \cdot \exp \left(\varphi_{A}\left(\mathcal{Y}^{\prime}\right)\right)
$$

where $\varphi_{A}\left(\mathcal{Y}^{\prime}\right)$ stands for the image of $\mathcal{Y}^{\prime}$ for the map $\varphi_{A}: A_{1} \otimes_{\mathbb{K}} \mathfrak{g}_{1}^{\prime} \longrightarrow A_{1} \otimes_{\mathbb{K}} \mathfrak{g}_{1}^{\prime \prime}$ obtained by scalar extension from $\left.\varphi\right|_{\mathfrak{g}_{1}^{\prime}}: \mathfrak{g}_{\mathbf{1}}^{\prime} \longrightarrow \mathfrak{g}_{\mathbf{1}}^{\prime \prime}$. As both $\phi_{+}$and $\exp \circ \varphi_{A} \circ\left(\left.\exp \right|_{A_{\mathbf{1}} \otimes \mathfrak{g}_{1}}\right)^{-1}$ are maps of $\mathcal{A}_{\mathbf{0}}$-manifolds, from (3.10) we deduce that $\Psi^{e}\left(\left(\phi_{+}, \varphi\right)\right)_{A}$ is a map of $\mathcal{A}_{\mathbf{0}}$-manifolds too, q.e.d.

\subsection{The new equivalences $(\mathrm{sHCp}) \cong($ Lsgrp $)$.}

In Section 3 we introduced two functors, denoted $\Psi^{\circ}$ and $\Psi^{e}$, from sHCp's to Lie supergroups. We will now show that both these two functors are quasi-inverse to the "natural" functor $\Phi$ considered in Section 2.2, so that (together with $\Phi$ ) they provide equivalences between the categories of sHCp's and of Lie supergroups.

We begin with the first half of our task, namely proving that $\Phi \circ \Psi \cong i d_{(\mathbf{s H C})}$ for $\Psi \in\left\{\Psi^{\circ}, \Psi^{e}\right\}$.

Proposition 3.5.1. Let $\Phi$ and $\Psi \in\left\{\Psi^{\circ}, \Psi^{e}\right\}$ be as in \$2.2.2 and in Theorems 3.2.6 and 3.4.6.

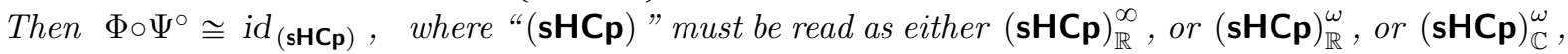
and $\Phi$ and $\Psi$ must be taken as working onto the corresponding types of Lie supergroups or sHCp's. 
Proof. This follows almost directly from definitions: we sketch the case of $\Psi^{\circ}$, that of $\Psi^{e}$ being similar.

Consider a super Harish-Chandra pair $\mathcal{P}:=\left(G_{+}, \mathfrak{g}\right)$, and let $G_{\mathcal{P}}^{\circ}=\Psi^{\circ}(\mathcal{P})$, so that $\left(\Phi \circ \Psi^{\circ}\right)(\mathcal{P})=$ $\Phi\left(G_{\mathcal{P}}^{\circ}\right)=\left(\left(G_{\mathcal{P}}^{\circ}\right)_{0}, \operatorname{Lie}\left(G_{\mathcal{P}}^{\circ}\right)\right)$. Then $\left(G_{\mathcal{P}}^{\circ}\right)_{0}=G_{+}$just by construction. In addition, the definition of $\operatorname{Lie}(G)$ and Proposition 3.2.4 together yield $\operatorname{Lie}\left(G_{\mathcal{P}}^{\circ}\right)=\operatorname{Lie}\left(G_{+} \times G_{-}^{<}\right)=\operatorname{Lie}\left(G_{+}\right) \oplus T_{e}\left(G_{-}^{<}\right)=\mathcal{L}_{\mathfrak{g}_{0}} \oplus \mathcal{L}_{\mathfrak{g}_{1}}=\mathcal{L}_{\mathfrak{g}}$ hence, identifying $\mathcal{L}_{\mathfrak{g}}=\mathfrak{g}$, simply $\operatorname{Lie}\left(G_{\mathcal{P}}^{\circ}\right)=\mathfrak{g}$. Thus $\left(\Phi \circ \Psi^{\circ}\right)(\mathcal{P})=\Phi\left(G_{\mathcal{P}}^{\circ}\right)=\left(\left(G_{\mathcal{P}}^{\circ}\right)_{0}, \operatorname{Lie}\left(G_{\mathcal{P}}^{\circ}\right)\right) \cong$ $\left(G_{+}, \mathfrak{g}\right)=\mathcal{P}$, i.e. $\Phi \circ \Psi^{\circ}$ acts on objects — up to natural isomorphisms — as the identity, q.e.d.

Now let $\left(\phi_{+}, \varphi\right): \mathcal{P}^{\prime}=\left(G_{+}^{\prime}, \mathfrak{g}^{\prime}\right) \longrightarrow\left(G_{+}^{\prime \prime}, \mathfrak{g}^{\prime \prime}\right)=\mathcal{P}^{\prime \prime}$ be a morphism of sHCp's and $\phi:=\Psi^{\circ}\left(\left(\phi_{+}, \varphi\right)\right)$ the corresponding (via $\left.\Psi^{\circ}\right)$ morphism of supergroups; we aim to prove that $\left(\Phi \circ \Psi^{\circ}\right)\left(\left(\phi_{+}, \varphi\right)\right)=\Phi(\phi)$ coincides, up to the natural isomorphisms $\left(\Phi \circ \Psi^{\circ}\right)\left(\mathcal{P}^{\prime}\right) \cong \mathcal{P}^{\prime}$ and $\left(\Phi \circ \Psi^{\circ}\right)\left(\mathcal{P}^{\prime \prime}\right) \cong \mathcal{P}^{\prime \prime}$, with $\left(\phi_{+}, \varphi\right)$ itself.

By definition $\Phi(\phi):=\left(\left.\phi\right|_{\left(G_{\mathcal{P}^{\prime}}\right)_{0}}, d \phi\right)$, and $\left.\phi\right|_{\left(G_{\mathcal{P}^{\prime}}\right)}=\left.\Psi^{\circ}\left(\left(\phi_{+}, \varphi\right)\right)\right|_{G_{+}^{\prime}}=\phi_{+}$by construction. Moreover, like in the proof of Theorem 3.2.6, using the factorizations $G_{\mathcal{p}^{\prime}}=G_{+}^{\prime} \times G_{-}^{\prime,<}$ and $G_{\mathcal{p}^{\prime \prime}}=G_{+}^{\prime \prime} \times G_{-}^{\prime \prime \prime<}$ we find that onto $T_{e}\left(G_{\mathcal{P}^{\prime}}\right)=T_{e}\left(G_{+}^{\prime}\right) \oplus T_{e}\left(G_{-}^{\prime,<}\right)=\mathfrak{g}_{\mathbf{0}} \oplus \mathfrak{g}_{\mathbf{1}}=\mathfrak{g}$ our $d \phi$ acts by $\left.d \phi\right|_{\mathfrak{g}_{0}}=d \phi_{+}=\left.\varphi\right|_{\mathfrak{g}_{\mathbf{0}}}$ and $\left.d \phi\right|_{\mathfrak{g}_{1}}=\left.\varphi\right|_{\mathfrak{g}_{1}} ;$ this gives $d \phi=\left.\left.d \phi\right|_{\mathfrak{g}_{\mathbf{0}}} \oplus d \phi\right|_{\mathfrak{g}_{1}}=\varphi_{\mathbf{0}} \oplus \varphi_{\mathbf{1}}=\varphi$, hence $\Phi(\phi):=\left(\left.\phi\right|_{\left(G_{\mathcal{P}^{\prime}}\right)_{\mathbf{0}}}, d \phi\right)=\left(\phi_{+}, \varphi\right)$.

The second half of our task is proving that $\Psi \circ \Phi$ is isomorphic to the identity functor on Lie supergroups:

Proposition 3.5.2. Let $\Phi$ and $\Psi \in\left\{\Psi^{\circ}, \Psi^{e}\right\}$ be as in §2.2.2 and in Theorems 3.2.6 and 3.4.6.

Then $\Psi^{\circ} \circ \Phi \cong i d_{(\text {Lsgrp })}$, where “(Lsgrp) " must be read as either $(\text { Lsgrp })_{\mathbb{R}}^{\infty}$, or $(\text { Lsgrp })_{\mathbb{R}}^{\omega}$, or $(\text { Lsgrp })_{\mathbb{C}}^{\omega}$, and $\Phi$ and $\Psi$ must be taken as working onto the corresponding types of Lie supergroups or $s H C p$ 's.

Proof. We begin again by looking at $\Psi^{\circ}$. Given a Lie supergroup $G$, set $\mathfrak{g}:=\operatorname{Lie}(G)$ and $\mathcal{P}:=\Phi_{g}(G)=$ $\left(G_{\mathbf{0}}, \mathfrak{g}\right)$. We look at $\Psi^{\circ}(\Phi(G))=\Psi^{\circ}(\mathcal{P})=: G_{\mathcal{P}}^{\circ}$, and prove that it is naturally isomorphic to $G$. For any $A \in(\mathbf{s a l g})_{\mathbb{k}}$, by abuse of notation we denote with the same symbol any $g_{0} \in G_{\mathbf{0}}(A)$ as belonging to $G(A)$ - via the embedding of $G_{\mathbf{0}}(A)$ into $G(A)$ - and as an element of $G_{\mathcal{P}}^{\circ}(A)$. With this convention, we easily see that Lemma 2.4.5 yields the following: there exists a unique group morphism $\phi_{A}: G_{\mathcal{P}}^{\circ}(A) \longrightarrow G(A)$ such that $\phi_{A}\left(g_{0}\right)=g_{0}$ for all $g_{0} \in G_{\mathbf{0}}(A)$ and $\phi_{A}((1+\eta Y))=(1+\eta Y)$ for all $\eta \in A_{\mathbf{1}}, Y \in \mathfrak{g}_{\mathbf{1}}$.

Due to the factorization (2.7) in Proposition 2.4.8, we have also that the morphism $\phi_{A}$ is surjective. Even more, the Global Splitting Theorem for $G$ (Theorem 2.4.10) and for $G_{\mathcal{P}}^{\circ}$ (Proposition 3.2.4) together imply that the morphism $\phi_{A}$ is also injective, hence it is a group isomorphism. Finally, all these $\phi_{A}$ 's are clearly natural in $A$, thus altogether they provide an isomorphism between $G_{\mathcal{p}}^{\circ}=\Psi^{\circ}(\Phi(G))$ and $G$, q.e.d.

The case of $\Psi^{e}$ is dealt with similarly, using the parallel results for $G_{\mathcal{P}}^{e}$ to those applied for $G_{\mathcal{P}}^{\circ}$ : namely, Lemma 2.4.5, formula (2.10) instead of (2.7), Proposition 3.4.4 instead of Proposition 3.2.4, etc.

To finish with, all the above jointly give the following, main result:

Theorem 3.5.3. Let $\Phi$ and $\Psi \in\left\{\Psi^{\circ}, \Psi^{e}\right\}$ be as in \$2.2.2 and in Theorems 3.2.6 and 3.4.6.

Then $\Psi^{\circ}$ and $\Psi^{e}$ are quasi inverse to $\Phi$, so they provide category equivalences

$$
(\mathbf{s H C p})_{\mathbb{R}}^{\infty} \cong(\text { Lsgrp })_{\mathbb{R}}^{\infty}, \quad(\text { sHCp })_{\mathbb{R}}^{\omega} \cong(\text { Lsgrp })_{\mathbb{R}}^{\omega}, \quad(\text { sHCp })_{\mathbb{C}}^{\omega} \cong(\text { Lsgrp })_{\mathbb{C}}^{\omega}
$$

\subsection{Linear case and representations.}

We shall now briefly discuss the fallout, in representation theory, of the existence of a an equivalence between Lie supergroups and sHCp's, in particular when realized via the functors $\Psi^{\circ}$ and $\Psi^{e}$ of $\S 3$.

3.6.1. The linear case. Assume that the Lie supergroup $G$ is linear, i.e. it embeds into some $\operatorname{GL}(V)$, where $V$ is a suitable superspace (in other words, there exists a faithful $G$-module $V$ ). Then $G_{0}$ and $\mathfrak{g}:=\operatorname{Lie}(G)$ embed into $\operatorname{End}(V)$, and the relations linking them such that $\left(G_{\mathbf{0}}, \mathfrak{g}\right)$ is a super Harish-Chandra pair are relations among elements of the unital, associative superalgebra $\operatorname{End}(V)$. Conversely, one can formally define a "linear sHCp" as being any $\operatorname{sHCp}\left(G_{\mathbf{0}}, \mathfrak{g}\right)$ such that both $G_{\mathbf{0}}$ and $\mathfrak{g}$ embed into some $\operatorname{End}(V)$, and the compatibility relations linking $G_{\mathbf{0}}$ and $\mathfrak{g}$ hold true as relations inside $\operatorname{End}(V)$ itself — mimicking what is 
done in [16], Definition 4.2.1(b), in the algebraic setup. The above then tells us that, if $G$ is linear, then its associated $\operatorname{sHCp} \Phi(G)=: \mathcal{P}$ is linear too - both being linearized via their faithful representation onto $V$.

On the other hand, let us start with a linear $\operatorname{sHCp}$, say $\mathcal{P}=\left(G_{+}, \mathfrak{g}\right)$ : so the latter is embedded (in the obvious sense) into the $\operatorname{sHCp}\left(\mathrm{GL}_{\mathbf{0}}(V), \mathfrak{g l}(V)\right)$ for some $V$. Thus for $A \in(\mathbf{W s a l g})_{\mathbb{K}}$ both $G_{+}(A)$ and $A \otimes_{\mathbb{K}} \mathfrak{g}$ are embedded into $(\operatorname{End}(V))(A)$, with relations among them - inside $(\operatorname{End}(V))(A)$ - induced by the very notion of linear sHCp. Now consider in $(\operatorname{End}(V))(A)$ all elements $\exp (\eta Y)=(1+\eta Y)-$ with $\eta \in A_{\mathbf{1}}, Y \in \mathfrak{g}_{\mathbf{1}}$ - that actually belong to $(\mathrm{GL}(V))(A)$ : moreover, $G_{+}(A) \subseteq(\mathrm{GL}(V))(A)$ too. Therefore, we can take inside $(\mathrm{GL}(V))(A)$ the subgroup $G_{\mathcal{P}}^{\circ, V}(A)$ generated by $G_{+}(A)$ and by all the $(1+\eta Y)$ 's.

A trivial check shows that the elements from $G_{+}(A)$ and the $\left(1+\eta_{i} Y_{i}\right)$ 's enjoy all relations that enter in the very definition $G_{\mathcal{P}}^{\circ}(A)$ : thus, there exists a (unique) group epimorphism $\phi_{A}^{\circ}: G_{\mathcal{P}}^{\circ}(A) \longrightarrow G_{\mathcal{P}}^{\circ, V}(A)$ such that $\phi_{A}^{\circ}\left(g_{+}\right)=g_{+}$and $\phi_{A}^{\circ}((1+\eta Y))=(1+\eta Y)$ for all $g_{+} \in G_{+}(A), \eta \in A_{\mathbf{1}}, Y \in \mathfrak{g}_{\mathbf{1}}$.

On the other hand, $G_{p}^{\circ, V}(A)$ acts faithfully on $V$ - indeed, it is "linearized by $V$ ". This allows one to show that the like of the Global Splitting Theorem — cf. Theorem 2.4.10 and Proposition 3.2.4 - does hold true for $G_{\mathcal{P}}^{\circ, V}(A)$, via the same analysis and arguments used in the proofs of those results but for one change: the linearization of $G_{\mathcal{P}}^{\circ, V}(A)$ has to replace the following key ingredients:

(1) in the proof of Theorem 2.4.10, one has that $G(A):=\coprod_{x \in|G|} \operatorname{Hom}_{(\mathbf{s a l g})_{\mathbb{K}}}\left(\mathcal{O}_{|G|, x}, A\right)$,

(2) in the proof of Proposition 3.2.4 (and lemmas before), $G_{\mathcal{P}}^{\circ}(A)$ is acting onto $V:=\operatorname{Ind}_{\mathfrak{g}_{0}}^{\mathfrak{g}}(\mathbf{1})-$ cf. (3.5). In fact, in both cases — of either $G(A)$ or $G_{\mathcal{p}}^{\circ}(A)$ - the group under examination is realized as a group of maps and these are rich enough to "separate (enough) points" so as to guarantee the uniqueness of factorization(s) that is the core part of the Global Splitting Theorem. In the case of $G_{\mathcal{P}}^{\circ, V}(A)$ instead, its built-in linearization provides a similar realization as "group of maps", and this again allows to separate enough points to get global splitting(s) for $G_{\mathcal{P}}^{\circ, V}(A)$ too. Finally, thanks to the Global Splitting Theorem for $G_{\mathcal{P}}^{\circ}(A)$ and for $G_{\mathcal{P}}^{\circ, V}(A)$, one can apply again the arguments used in the proof of Proposition 3.5.2 and successfully prove that the above group (epi)morphism $\phi_{A}^{\circ}: G_{\mathcal{P}}^{\circ}(A) \longrightarrow G_{\mathcal{P}}^{\circ, V}(A)$ is also injective, hence it is an isomorphism.

By construction all these isomorphisms $\phi_{A}^{\circ}$ are natural in $A$, hence they give altogether a functor isomorphism $\phi^{\circ}: G_{\mathcal{P}}^{\circ} \cong G_{\mathcal{P}}^{\circ, V}$. Therefore $G_{\mathcal{P}}^{\circ} \cong G_{\mathcal{P}}^{\circ, V}$, which means that we found a different, concrete realization of $G_{\mathcal{P}}^{\circ}$, that is now constructed explicitly as the linear Lie supergroup $G_{\mathcal{P}}^{\circ, V}$.

In a parallel way, still starting with a linear $\operatorname{sHCp} \mathcal{P}=\left(G_{+}, \mathfrak{g}\right)$ embedded into $\left(\operatorname{GL}_{\mathbf{0}}(V), \mathfrak{g l}(V)\right)$, we consider in $(\operatorname{End}(V))(A)$ all elements of the form $\exp (\mathcal{Y})$ with $\mathcal{Y} \in A_{\mathbf{1}} \otimes_{\mathbb{K}} \mathfrak{g}_{\mathbf{1}}$ and then take the subgroup $G_{\mathcal{P}}^{e, V}(A)$ of $(\mathrm{GL}(V))(A)$ generated by $G_{+}(A)$ and by all the $\exp (\mathcal{Y})$ 's. Acting like above we find that there exists a group epimorphism $\phi_{A}^{e}: G_{\mathcal{P}}^{e}(A) \longrightarrow G_{\mathcal{P}}^{e, V}(A)$ such that $\phi_{A}^{e}\left(g_{+}\right)=g_{+}, \quad \phi_{A}^{e}(\exp (\mathcal{Y}))=\exp (\mathcal{Y})$, for all $g_{+} \in G_{+}(A)$ and $\mathcal{Y} \in A_{\mathbf{1}} \bigotimes_{\mathbb{K}} \mathfrak{g}_{\mathbf{1}}$. Even more, still by the same method as above we find that all these $\phi_{A}^{e}$ 's are in fact isomorphisms, natural in $A$, hence they define a functor isomorphism $\phi^{e}: G_{\mathcal{P}}^{e} \cong G_{\mathcal{P}}^{e, V}$. This gives yet another concrete realization of $G_{\mathcal{P}}^{e}$, now explicitly realized as the linear Lie supergroup $G_{\mathcal{P}}^{e, V}$.

Finally, the very construction also shows, indeed, that $G_{\mathcal{P}}^{\circ, V}=G_{\mathcal{P}}^{e, V}$.

3.6.2. Induction from $\mathbf{G}_{\mathbf{0}}$ to $\mathbf{G}$. Let $G$ be a supergroup (of any type), with classical subsupergroup $G_{\mathbf{0}}$,

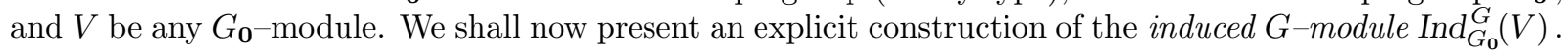

The $G_{\mathbf{0}^{-}}$-module $V$ is also a $\mathfrak{g}_{\mathbf{0}}$-module, hence one has the induced $\mathfrak{g}$-module $\operatorname{Ind} d_{\mathfrak{g}_{\mathbf{0}}}^{\mathfrak{g}}(V)$, that one can realize as $\operatorname{Ind}_{\mathfrak{g}_{\mathbf{0}}}^{\mathfrak{g}}(V)=\operatorname{Ind}_{U(\mathfrak{g})}^{U(\mathfrak{g})}(V)=U(\mathfrak{g}) \otimes_{U\left(\mathfrak{g}_{\mathbf{0}}\right)} V$. This bears also a unique structure of $G_{\mathbf{0}^{-}}$module which is compatible with the $\mathfrak{g}$-action and coincides with the original $G_{\mathbf{0}^{-}}$-action on $\mathbb{K} \otimes_{U\left(\mathfrak{g}_{0}\right)} V \cong V$ given from scratch. Indeed, we can describe explicitly this $G_{0^{-}}$action, as follows. First, definitions give $\operatorname{Ind}_{\mathfrak{g}_{0}}^{\mathfrak{g}}(V)=$ $U(\mathfrak{g}) \otimes_{U\left(\mathfrak{g}_{\mathbf{0}}\right)} V=\bigwedge \mathfrak{g}_{1} \otimes_{\mathbb{K}} V$ - see $(3.4)$ — with the $\mathfrak{g}_{\mathbf{0}}$-action given by $x .(y \otimes v)=\operatorname{ad}(x)(y) \otimes v+y \otimes(x . v)$ for $x \in \mathfrak{g}_{0}, y \in \wedge \mathfrak{g}_{1}, v \in V$, where by ad we denote the unique $\mathfrak{g}_{0}$-action on $\bigwedge \mathfrak{g}_{1}$ by algebra derivations induced by the adjoint $\mathfrak{g}_{0}$-action on $\mathfrak{g}_{1}$. Second, this action integrates to a (unique) $G_{0^{-}}$action given by $g_{0} \cdot(y \otimes v):=\operatorname{Ad}\left(g_{0}\right)(y) \otimes\left(g_{0} \cdot v\right)$ for $g_{0} \in G_{\mathbf{0}}, y \in \wedge \mathfrak{g}_{\mathbf{1}}, v \in V$, where we write $A d$ for the $G_{\mathbf{0}^{-}}$action on $\bigwedge \mathfrak{g}_{1}$ by algebra automorphisms induced by the adjoint $G_{0^{-}}$action on $\mathfrak{g}_{\mathbf{1}}$.

The key point is that the above $G_{0^{-}}$-action and the built-in $\mathfrak{g}$-action on $\operatorname{Ind}_{\mathfrak{g}_{0}}^{\mathfrak{g}}(V)$ are compatible, in that they make $\operatorname{Ind}_{\mathfrak{g}_{0}}^{\mathfrak{g}}(V)$ into a module for the $\operatorname{sHCp} \mathcal{P}:=\left(G_{\mathbf{0}}, \mathfrak{g}\right)=\Phi(G)$. Then for $\Psi \in\left\{\Psi^{\circ}, \Psi^{e}\right\}$, since $\Psi\left(\left(G_{\mathbf{0}}, \mathfrak{g}\right)\right) \cong G$, clearly $\operatorname{Ind}_{\mathfrak{g}_{\mathbf{0}}}^{\mathfrak{g}}(V)$ bears a unique structure of $G$-module which corresponds to the previous $\mathcal{P}$-action. In down-to-earth terms what happens is the following. The action of $\mathcal{P}:=\left(G_{\mathbf{0}}, \mathfrak{g}\right)=\Phi(G)$ onto $W:=\operatorname{Ind}_{\mathfrak{g}_{0}}^{\mathfrak{g}}(V)$ is given by the $G_{\mathbf{0}^{-}}$action (induced by that on $V$ ) and a compatible $\mathfrak{g}$-action. Then for 
any $A \in(\mathbf{W s a l g})_{\mathbb{K}}$ we have also that all of $A_{\mathbf{1}} \otimes_{\mathbb{K}} \mathfrak{g}_{\mathbf{1}}$ "acts" onto $A \otimes_{\mathbb{K}} W$ : thus well-defined operators $(1+\eta Y)$ and $\exp (\mathcal{Y})$ - with $(\eta, Y) \in A_{\mathbf{1}} \times \mathfrak{g}_{\mathbf{1}}, \mathcal{Y} \in A_{\mathbf{1}} \otimes_{\mathbb{K}} \mathfrak{g}_{\mathbf{1}}$ - exist in $(\mathrm{GL}(W))(A)$. One checks that these $(1+\eta Y)$ 's, or the $\exp (\mathcal{Y})$ 's, altogether enjoy among themselves and with the operators given by the $G_{\mathbf{0}^{-}}$-action all relations that enter in the definition of either $\Psi^{\circ}(\mathcal{P}):=G_{\mathcal{P}}^{\circ}$ or $\Psi^{e}(\mathcal{P})=G_{\mathcal{P}}^{e}$ - thus in both cases we get an action of $\Psi(\mathcal{P})$ on $W$, extending the initial one by $G_{0}$ : but $\Psi(\mathcal{P})=G$, so we are done.

Thus we define as $\operatorname{Ind}_{G_{0}}^{G}(V)$ the space $W:=\operatorname{Ind}_{\mathfrak{g}_{0}}^{\mathfrak{g}}(V)$ endowed with this $G$-action: one checks that this construction is functorial in $V$ and has the universal property making it adjoint of "restriction" (from $G$-modules to $G_{\mathbf{0}^{-}}$-modules), so it can be correctly called "induction" functor. In addition, if the $G_{0^{-}}$-module $V$ is faithful then the $G$-module $\operatorname{Ind}_{G_{0}}^{G}(V)$ is faithful too: this means that if $G_{0}$ is linearizable, then $G$ is linearizable too; in fact, from a linearization of $G_{\mathbf{0}}$ we can construct (via induction) a linearization of $G$ too.

\section{References}

[1] A. Alldridge, J. Hilgert, T. Wurzbacher, Singular superspaces, Math. Z. 278 (2014), no. 1-2, 441-492.

[2] A. Alldridge, M. Laubinger, Infinite-dimensional supermanifolds over arbitrary base fields, Forum Math. 24 (2012), no. 3, 565-608.

[3] L. Balduzzi, C. Carmeli, R. Fioresi, A comparison of the functors of points of supermanifolds, J. Algebra Appl. 12 (2013), no. 3, 1250152, pp. 41.

[4] F. A. Berezin, Introduction to superanalysis, Edited by A. A. Kirillov. D. Reidel Publishing Company, Dordrecht (Holland), 1987.

[5] W. Bertram, A. Souvay, A general construction of Weil functors, Cah. Topol. Géom. Différ. Catég. 55 (2014), no. 4, 267-313.

[6] H. Boseck, Affine Lie supergroups, Math. Nachr. 143 (1989), 303-327.

[7] C. Carmeli, L. Caston, R. Fioresi, Mathematical Foundations of Supersymmetry (with an appendix by I. Dimitrov), EMS Series of Lectures in Mathematics 15, Eur. Math. Soc., Zürich, 2011.

[8] C. Carmeli, R. Fioresi, Super Distributions, Analytic and Algebraic Super Harish-Chandra pairs, Pacific J. Math. 263 (2013), no. 1, 29-51.

[9] C. Carmeli, R. Fioresi, V. S. Varadarajan, The Exponential of Nilpotent Supergroups in the Theory of Harish-Chandra Representations, in: Geometric science of information, 188-195, Lecture Notes in Comput. Sci., 11712, Springer, Cham, 2019.

[10] P. Deligne, J. Morgan, Notes on supersymmetry (following J. Bernstein), in: "Quantum fields and strings: a course for mathematicians", Vol. 1, 2 (Princeton, NJ, 1996/1997), 41-97, Amer. Math. Soc., Providence, RI, 1999.

[11] R. Fioresi, F. Gavarini, Chevalley Supergroups, Memoirs AMS 215, no. 1014 (2012).

[12] R. Fioresi, F. Gavarini, On the construction of Chevalley supergroups, in: S. Ferrara, R. Fioresi, V. S. Varadarajan (eds.), "Supersymmetry in Mathematics \& Physics", UCLA Los Angeles, U.S.A. 2010, Lecture Notes in Math. 2027, Springer-Verlag, Berlin-Heidelberg, 2011, pp. 101-123.

[13] F. Gavarini, Chevalley Supergroups of type $D(2,1 ; a)$, Proc. Edinb. Math. Soc. (2) 57 (2014), no. 2, 465-491.

[14] F. Gavarini, Algebraic supergroups of Cartan type, Forum Math. 26 (2014), no. 5, 1473-1564.

[15] F. Gavarini, Corrigendum to "Algebraic supergroups of Cartan type", Forum Math. 28 (2016), no. 5, 1005-1009. 
[16] F. Gavarini, Global splittings and super Harish-Chandra pairs for affine supergroups, Trans. Amer. Math. Soc. 368 (2016), no. 6, 3973-4026.

[17] F. Gavarini, Lie supergroups vs. super Harish-Chandra pairs: a new equivalence, on-line preprint arXiv:1609.02844 [math.RA] (2016).

[18] B. Kostant, Graded manifolds, graded Lie theory, and prequantization, in: Differential geometrical methods in mathematical physics (Proc. Sympos., Univ. Bonn, Bonn, 1975), 177-306, Lecture Notes in Math. 570, Springer, Berlin, 1977.

[19] J.-L. Koszul, Graded manifolds and graded Lie algebras, Proceedings of the international meeting on geometry and physics (Florence, 1982), 71-84, Pitagora, Bologna, 1982.

[20] D. A. Leites, Introduction to the theory of supermanifolds, Russian Math. Surveys 35 (1980), 1-64.

[21] A. Masuoka, Harish-Chandra pairs for algebraic affine supergroup schemes over an arbitrary field, Transform. Groups 17 (2012), no. 4, 1085-1121.

[22] A. Masuoka, T. Shibata, Algebraic supergroups and Harish-Chandra pairs over a commutative ring, Trans. Amer. Math. Soc. 369 (2017), no. 5, 3443-3481.

[23] V. Molotkov, Infinite-dimensional and colored supermanifolds, J. Nonlinear Math. Phys. 17 (2010), suppl. 1, 375-446.

[24] V. S. Varadarajan, Supersymmetry for mathematicians: an introduction, Courant Lecture Notes 1, AMS, 2004

[25] E. G. Vishnyakova, On complex Lie supergroups and homogeneous split supermanifolds, Transform. Groups 16 (2011), no. 1, 265-285. 